\title{
Geodesics in semi-Riemannian Manifolds: Geometric Properties and Variational Tools
}

\author{
Anna Maria Candela*, Miguel Sánchez ${ }^{\dagger}$
}

\begin{abstract}
Geodesics become an essential element of the geometry of a semi-Riemannian manifold. In fact, their differences and similarities with the (positive definite) Riemannian case, constitute the first step to understand semi-Riemannian Geometry. The progress in the last two decades has become impressive, being especially relevant the systematic introduction of (infinite-dimensional) variational methods.

Our purpose is to give an overview, from refinements of classical results to updated variational settings. First, several properties (and especially completeness) of geodesics in some ambient spaces are studied. This includes heuristic constructions of compact incomplete examples, geodesics in warped, GRW or stationary spacetimes, properties in surfaces and spaceforms, or problems on stability of completeness.

Then, we study the variational framework, and focus on two fundamental problems of this approach, which regards geodesic connectedness. The first one deals with a variational principle for stationary manifolds, and its recent implementation inside Causality Theory. The second one concerns orthogonal splitting manifolds, and a reasonably selfcontained development is provided, collecting some steps spread in the literature.
\end{abstract}

Mathematics Subject Classification (2000). Primary 53C22, 58E10; Secondary 53C50, 53C $80,58 \mathrm{E} 05$.

Keywords. Geodesic, semi-Riemannian manifold, geodesic completeness, Causality Theory, Clifton-Pohl torus, Misner's cylinder, density of incompleteness in tori, homogeneous manifold spaceform, stability of completeness, geodesic in surface, Generalized Robertson-Walker (GRW) spacetime, warped-completeness, singularity theorem, action functional, Palais-Smale condition, geodesic connectedness, static spacetime, stationary spacetime, orthogonal splitting spacetime, globally hyperbolic spacetime, LjusternikSchnirelman Theory, Relative Category Theory.

\footnotetext{
*Supported by M.I.U.R. (research funds ex $40 \%$ and $60 \%$ ).

†Supported by MEC Grant MTM-2004-04934-C04-01.
} 


\section{Contents}

1 Introduction 2

2 First properties $\quad 5$

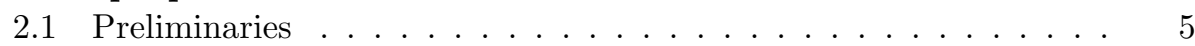

2.2 Special properties of geodesics in spacetimes depending on their causal character 7

2.3 Conformal changes . . . . . . . . . . . . . . . . . . 10

3 An overview on geodesics in different ambient manifolds and geodesic completeness 11

3.1 First results . . . . . . . . . . . . . . . . . . 11

3.2 Completeness under conformal svmmetries . . . . . . . . . . . . . 14

3.3 Heuristic construction of incomplete examples . . . . . . . . . . . . 16

3.4 Surfaces . . . . . . . . . . . . . . . . . . . 19

3.5 Influence of curvature . . . . . . . . . . . . . . . . . 21

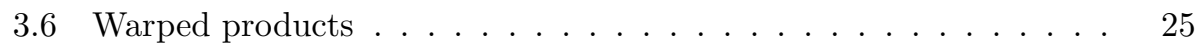

3.7 GRW spacetimes $\ldots \ldots \ldots \ldots \ldots$

3.8 Stationarv spacetimes $\ldots \ldots \ldots \ldots$. . . . . . . . . . 31

4 Variational approaches in Lorentzian manifolds 33

4.1 The Riemannian framework for geodesics connecting two points . . 34

4.2 Variational principles for static and stationarv spacetimes: extrinsic and intrinsic approaches 39

4.3 Time dependent metrics and saddle critical points . . . . . . . 46

\section{Introduction}

Geodesics become an essential ingredient of semi-Riemannian Geometry, as so they are for the (positive definite) Riemannian one. In the indefinite case, two important difficulties arise:

(1) there are simple questions which become elementary in the Riemannian case but they are open in general. For some of them, it is not clear which type of techniques will work - say, just a simple brilliant idea or a full specific theory;

(2) in the Lorentzian case, Causality is a specific theory fully adapted to its particularities. So, other sophisticated theories which may work in the Riemannian case, must be implemented carefully in the framework of Causality. This may be difficult, but it makes possible to obtain powerful results. In fact, higher order indexes may be intractable, as there is no tool as Causality.

Accordingly, here our aim is twofold. The first one, developed in Section 3 is to provide a brief overview on geometric properties of geodesics in the general semi-Riemannian case, stressing the differences and similarities with the Riemannian one. Even though many of them are simple items, they may involve very different techniques, and pose open questions at a primary level, whose strategy of solution is hard to predict. For example, as far as we know, the following question is open: must a compact semi-Riemannian manifold globally conformal 
to a constant curvature one be complete? Here, the reader will find related results as: (i) compact Lorentzian manifolds of constant curvature are complete (see Theorem [3.30), (ii) lightlike completeness is conformally invariant in the compact case (see Theorem 2.2), (iii) in locally symmetric spaces the three types of causal completeness are equivalent (see Theorem 3.3), (iv) there are difficulties to find independence of causal completeness in the compact case (see Remarks 3.27 3.2), and $(v)$ there exists a relation between the completeness of conformally related compact semi-Riemannian manifolds, the causal independence of completeness and the completeness of warped products (see Remark 3.45). These five items have been studied by means of very different techniques, some of them elementary and some other ones highly sophisticated. It is not clear which type of reasonings can solve the open question but, at any case, the answers to these type of problems will yield an advance in our knowledge of semi-Riemannian Geometry. In order to be specific, most of the overview in Section 3 concerns geodesic completeness. Nevertheless, the reader is introduced in the behaviour of geodesics of different ambients: locally symmetric, homogeneous, spaceforms, warped products, stationary... The reader is expected to have a basic knowledge of Riemannian Geometry, and we hope he/she will learn which Riemannian results and tools still hold or can be adapted to the indefinite case. So, we choose a heuristic approach in topics such as the counterexamples to completeness (see Subsection 3.3) or the role of curvature in singularity theorems (see Subsection 3.5). Some of the topics on geodesics covered here, can be complemented with other properties of the manifolds, as those ones surveyed in [70].

Our second main aim, developed in Section 4 is to give an overview of the infinite-dimensional variational setting for geodesics in Lorentzian manifolds introduced at the end of 80's by Benci, Fortunato, Giannoni and Masiello (see, e.g., 12, 14, 47). Even though this approach has been explained in book format by Masiello [1], remarkable progress in the foundations of the theory has been carried out since then. First, as commented above, the interplay between the variational theory and Causality is necessary for the development of the approach in all its extent. This full interplay has been achieved only very recently in [24, and just for the most simple "fundational problem" of the theory, i.e, geodesic connectedness of static and stationary manifolds. We think that further results in this direction may be of big interest for both, the variational analyst's and Lorentzian geometer's viewpoints. On the other hand, regarding the "second fundational problem", i.e., geodesic connectedness in splitting type manifolds, we emphasize that, even though the core of the approach is explained in references as the book [61, the full details were filled later, and are spread in several papers published along the last decade. So, we explain it in a reasonably self-contained way.

The variational approach starts by recalling that, whenever $(M, g)$ is a semiRiemannian manifold, its geodesics can be found as critical points of the action functional

$$
f(\gamma)=\int_{0}^{1} g(\gamma)\left[\gamma^{\prime}, \gamma^{\prime}\right] d s,
$$

where $\gamma:[0,1] \rightarrow M$ are curves taken in a suitable manifold of functions (for more 
details, see later on). If $M$ is a Riemannian manifold, i.e., its metric $g$ has index $s_{0}=0$, functional $f$, now named energy functional, can be directly studied by means of classical variational tools as it is positive, hence, bounded from below (see Section 4.1).

But, more in general, if the semi-Riemannian metric $g$ has index $s_{0} \geq 1$ the corresponding functional $f$ is strongly indefinite (i.e., unbounded both from above and from below, even up to compact perturbations) with critical points having infinite Morse index. Thus, different methods and suitable "tricks" are needed and, at least in the Lorentzian case, we can distinguish two different variational approaches which allow one to overcome such a problem (see the book [61] or the survey [89]):

(a) to transform the indefinite problem on a Lorentzian manifold in a subtler (hopefully bounded from below) problem on a Riemannian manifold;

(b) to study directly the strongly indefinite functional $f$ but by making use of suitable (essentially finite-dimensional) "approximating" problems.

Furthermore, the choice of the right manifold of curves where functional $f$ is defined, depends on the different geometric problem studied, and impose specific "boundary" properties:

- geodesics joining two fixed points (see Section 4.1),

- closed geodesics (see, e.g., 28, 60] and references therein),

- geodesics connecting two given submanifolds (see, e.g., 65] or also [26] and references therein),

- T-periodic trajectories (see, e.g., the pioneer papers [12, 50, the survey [20] or [6], and references therein).

Moreover, one can use variational tools also in order to find geodesics with given causal character (for the timelike ones, see, e.g., [5] and also references in 21, for the lightlike ones, see the Fermat principle in [41, 42]).

The difficulty of the interplay between variational methods and Causality makes that, in some particular cases, non-variational approaches may yield more accurate results (see, for example, 39, 40] for geodesic connectedness, 90] for closed geodesics, or [86, 88, for $T$-periodic trajectories). Nevertheless, one may expect that, as we have commented for the stationary case, the strongest results will be obtained when the variational tools are fully implemented in Causality Theory.

Here, for simplicity, we want just to outline how to manage the previous approaches (a), (b) in a model case. More precisely, after the introductory Riemannian case in Subsection 4.1 we study geodesic connectedness: in stationary spacetimes (method (a)) in Subsection 4.2 and in orthogonal splitting spacetimes (method (b)) in Subsection 4.3 


\section{First properties}

Most of the material in this preliminary section is well-known. In the Subsection 2.1. essentially, notation and first definitions are given. In Subsection 2.2 first we summarize maximizing properties of timelike and lightlike geodesics in the Lorentzian case (but we refer to [63] in these proceedings for much more detailed results). Spacelike geodesics, including some remarkable properties of their conjugate points, are also considered here. The transformation of the Levi-Civita connection and geodesics under conformal changes (including implications for lightlike geodesics) are studied in Subsection 2.3. and will be widely used throughout Section 3

2.1. Preliminaries. Usual notation and conventions, essentially compatible with standard books as [11] or [67, will be used. We refer also to these references for detailed proofs of the basic properties collected in the present subsection. As we have pointed out, the interplay with Causality will be essential in the Lorentzian case and, so, the contribution to these proceedings [63 will be frequently invoked for background material on Causality.

Definition 2.1. A semi-Riemannian manifold is a smooth manifold $M$, of dimension $n_{0} \geq 1$, endowed with a non-degenerate metric $g: M \rightarrow T^{*} M \otimes T^{*} M$ of constant index $s_{0}$.

In the case $s_{0}=0$ the manifold is called Riemannian (or positive definite), if $s_{0}=n_{0}$ is called negative definite, if $0<s_{0}<n_{0}$ is called indefinite and, in this case, if $s_{0}=1\left(n_{0}>1\right)$ is called Lorentzian.

By smooth we mean $C^{r_{0}} ; r_{0}=+\infty$ will be assumed for simplicity for all the elements, except when otherwise is stated explicitly. $M$ will be also assumed (in addition to Hausdorff, as usual) connected, except if otherwise is specified; thus, the constancy of $s_{0}$ can be deduced of the non-degeneracy ${ }^{1}$ of $g$. The notion of "causal character" for a tangent vector $v \in T M$ in Lorentzian Geometry, which comes from General Relativity, is extended here and, so, $v$ is called timelike (resp., lightlike; causal; spacelike) depending on if $g(v, v)<0$ (resp. if $g(v, v)=0$ and $v \neq 0 ; v$ is either timelike or lightlike, i.e., $g(v, v) \leq 0$ and $v \neq 0 ; g(v, v)>0$ ). Vector 0 does not lie in any of these types, even though sometimes is useful to regard it as spacelike (see [67]); according to [63, a null vector will be either timelike or 0 . These causal characters are naturally extended to curves and submanifolds. We will put $|v|=\sqrt{|g(v, v)|}$ and the length of a curve $\gamma$ is the integral of $\left|\gamma^{\prime}\right|$.

Recall that the existence and uniqueness of the Levi-Civita connection $\nabla$ depends only on the non-degeneracy of the metric $g$ and, therefore, it can be deduced in the semi-Riemannian case as in the Riemannian one. Associated to $\nabla$ there is a covariant derivative $D / d s$ and parallel transport (which is an isometry, too). Moreover, a smooth curve $\gamma: I \rightarrow M$ is a geodesic if its vector field is parallel

\footnotetext{
${ }^{1}$ This non-degeneracy also implies paracompactness and, as $M$ is connected, the second axiom of numerability for the topology (see 63 for references).
} 
or, equivalently, its acceleration vanishes:

$$
\frac{D \gamma^{\prime}}{d s}(s)=0 \quad \text { for all } s \in I .
$$

If $\gamma$ is a geodesic, $g\left(\gamma^{\prime}, \gamma^{\prime}\right)$ is a constant. Thus, the causal character of a geodesic is defined as timelike, lightlike etc. according to the constant causal character of its velocity. By taking an orthonormal basis $B_{p}=\left(v_{1}, \ldots, v_{n_{0}}\right)$ at $T_{p} M$, that is, satisfying

$$
g_{p}\left(v_{i}, v_{j}\right)=\epsilon_{i} \delta_{i j}, \quad \epsilon_{i}=-1, \text { if } i=1, \ldots, s_{0}, \quad \epsilon_{i}=1, \text { if } i=s_{0}+1, \ldots, n_{0}
$$

one obtains a natural isometry with $\mathbb{R}_{s_{0}}^{n_{0}}$, that is, $\mathbb{R}^{n}$ endowed with the natural product of index $s_{0}$. In the Lorentzian case, the semi-Riemannian manifold $\mathbb{R}_{s_{0}}^{n_{0}}$ will be the Lorentz-Minkowski spacetime, denoted by $\mathbb{L}^{n_{0}}$.

As for any affine connection, the differential at 0 of the exponential map, exp,

$$
\left(d \exp _{p}\right)_{0}: T_{0}\left(T_{p} M\right) \rightarrow T_{p} M
$$

is the identity up to natural identifications. Thus, any point $p \in M$ admits a starshaped neighborhood $U$ ( $\exp _{p}^{-1}$ becomes well defined on $U$ and its image is a starshaped neighborhood of $0 \in T_{p} M$ in the usual sense), a normal neighborhood (any chart $\left(U, \varphi \circ \exp _{p}^{-1}\right)$, where $U$ is starshaped and $\varphi: T_{p} M \rightarrow \mathbb{R}_{s_{0}}^{n_{0}}$ a linear isometry) and a convex neighborhood (a normal neighborhood of all its points), see [67. Proposition 5.7]. Gauss Lemma also makes sense, and can be proved in a similar way as in the Riemannian case (see [11 Theorem 10.18], 67, Lemma 5.1]): if $p \in M, 0 \neq x \in T_{p} M$ and $v_{x}, w_{x} \in T_{x}\left(T_{p} M\right)$ with $v_{x}$ collinear with $x$, then:

$$
g_{p}\left(\left(d \exp _{p}\right)_{x}\left(v_{x}\right),\left(d \exp _{p}\right)_{x}\left(w_{x}\right)\right)=\left\langle v_{x}, w_{x}\right\rangle
$$

where $\langle\cdot, \cdot\rangle$ is the scalar product in $T_{x}\left(T_{p} M\right)$ naturally induced by $g_{p}$.

The notion of conjugate point $q$ of a point $p$ along a geodesic $s \mapsto \gamma(s)=$ $\exp _{p}(s v), v \in T_{p} M$ (i.e., $q=\exp _{p}\left(s_{q} v\right)$ where $s_{q} v$ is a critical point of $\left.\exp _{p}\right)$ and its multiplicity (dimension of the kernel of $d \exp _{p}$ at $s_{q} v$ ), is a natural extension of the usual Riemannian definition. Nevertheless, their properties may be very different, as we will see below. Jacobi equation (and its solutions, the Jacobi fields) is also defined in the semi-Riemannian case as a formal extension of the Riemannian one, that is: $D J^{\prime} / d s=-R\left(J, \gamma^{\prime}\right) \gamma^{\prime}$, where $R$ is the curvature tensor under the convention $R(X, Y)=\left[\nabla_{X}, \nabla_{Y}\right]-\nabla_{[X, Y]}$ for all vector fields $X, Y \in \mathfrak{X}(M)$. Moreover, Jacobi fields on $\gamma$ correspond to variational fields through longitudinal geodesics, and the multiplicity of a conjugate point $q$ to $p$ along $\gamma$ is equal to the dimension of the space of Jacobi vector fields on $\gamma$ which vanishes on $p, q$ (see 67 . Propositions 8.6, 10.10]).

The index form of a non-lightlike geodesic $\gamma:[a, b] \rightarrow M$ is analogously defined as:

$$
I(V, W)=\nu \int_{a}^{b}\left(g\left(\frac{D V}{d s}, \frac{D W}{d s}\right)-g\left(R\left(V, \gamma^{\prime}\right) \gamma^{\prime}, W\right)\right) d s
$$


where $\nu$ is a non-null constant which depends on the speed $\left|\gamma^{\prime}\right|$, and the causal character of $\gamma$ (and is chosen with different values for different authors), and $V, W$ belong to $\mathfrak{X}^{\perp}(\gamma)$, i.e., the space of all the (piecewise) smooth vector fields on $\gamma$ with vanishing endpoints pointwise orthogonal to $\gamma^{\prime}$. For lightlike geodesics, the index form $I$ can be defined formally as above, but recall: (a) the multiplicity of a conjugate point along a non-lighlike geodesic $\gamma$ can be obtained as the dimension of the radical (nullspace) of $I$ (see 67. Corollary 10.12]), (b) in order to extend this result to the lightlike case, $I$ is replaced by the quotient index form $\bar{I}$. With this aim, one first define the quotient space $\overline{\mathfrak{X}}^{\perp}(\gamma)$ obtained by identifying each two $X, Y \in \mathfrak{X}^{\perp}(\gamma)$ if $X-Y$ is pointwise collinear to $\gamma^{\prime}$. Then, $\bar{I}$ is defined as the

bilinear form on $\overline{\mathfrak{X}}^{\perp}(\gamma)$ obtained by inducing naturally $I$ (see [63]). Even more, in the Lorentzian case, a Morse Index Theorem holds for both, the index form $I$ on timelike geodesics and the quotient index form on lightlike geodesics (see 11 . Chapter 10]).

At last, let us recall that a spacetime $M$ is globally hyperbolic if there exists a (smooth) spacelike Cauchy hypersurface $\mathcal{S}$ in $M$, i.e., a subset which is crossed exactly once by any inextendible timelike curve 63 .

\subsection{Special properties of geodesics in spacetimes depending} on their causal character. We will mean by co-spacelike any geodesic such that the orthogonal of its velocity is a spacelike subspace at each point, that is: all the geodesics in the Riemannian case and timelike geodesics in the Lorentzian one. They present similar properties of extremization (minimization or maximization) for the "distance" $d$ associated to the metric $g$. By such a distance we mean the true canonical distance associated to $g$, if $g$ is Riemannian (i.e., (4.3) below), but the time-separation in the Lorentzian one. Concretely (as in 63]), if $g$ is Lorentzian, we assume in the present subsection that it admits a time-orientation (i.e., a continuous choice of causal cones, which will be called future cones). $(M, g)$, with the additional choice of a time-orientation, is a spacetime, and the time-separation (or Lorentzian distance) is defined for any $p, q \in M$ as the supremum $d(p, q)$ of the lengths of the future--directed causal curves from $p$ to $q$.

As a first definition, a causal (resp. Riemannian) geodesic with endpoints $p, q$ will be called maximizing (resp. minimizing) when its length is equal to the maximum between $d(p, q)$ and $d(q, p)$ (resp. $d(p, q)$ ). Recall that, even though $d$ is not symmetric in a general spacetime, the notation will be simplified below writing just $d(p, q)$ for the maximum between $d(p, q)$ and $d(q, p)$. The extremizing properties of co-spacelike geodesics for $d$ can be deduced by means of a standard study of the index form. Even more, many of them can be extended to lightlike geodesics as a specific case.

Next, we sketch a very rough summary. We emphasize that, in the Lorentzian case, these properties depend heavily on the causal structure of the spacetime and, so, we refer to the contribution 63 for detailed definitions and properties. Here, our purpose is just to stress the similitudes and differences of the extremizing properties of timelike geodesics and Riemannian geodesics, and how they can be extended to the lightlike case (see [63. Theorems 2.26, 2.27] for precise statements). 
So, in the next paragraphs, the extremizing properties will be stated first for timelike geodesics, and the corresponding Riemannian property is also pointed out. Then, the lightlike case will be studied. Finally, some comments on the spacelike case will be added.

Timelike and co-spacelike geodesics. It is well known that conjugate points along a timelike (resp. Riemannian) geodesic in a Lorentzian (resp. Riemannian) manifold cannot have points of accumulation. Even more:

(1) Any timelike geodesic maximizes locally $d$ in a similar way as any Riemannian geodesic minimizes locally its corresponding $d$. Nevertheless, there are two important differences:

- Riemannian geodesics minimize locally length among all the smooth curves connecting two fixed points $p, q$. Nevertheless, the timelike ones maximize only among the causal curves connecting $p, q$;

- the Riemannian minimizing property happens for both, the restriction $\left.d\right|_{U}$ of the distance $d$ on $M$ to some small neighborhod $U$, and the distance $d_{U}$ naturally associated to the restriction $g_{U}$ of the metric to a suitable neighborhood $U$ (in fact, for the latter, $U$ can be taken as any convex neighborhood). In general, the maximizing property for timelike geodesics happens only in the latter sense. It happens in the former one in strongly causal spacetimes (in particular, in globally hyperbolic ones). Recall that, for example, there are spacetimes (totally vicious ones) with $d \equiv+\infty$ and, thus, so it does $\left.d\right|_{U}$.

(2) Let $\gamma:[a, b] \rightarrow M$ be any timelike (resp. Riemannian) geodesic which connects two points $p, q \in M$ non-conjugate along $\gamma$. Then: $\gamma$ will have strictly maximum (resp. minimum) length among close curves connecting $p, q$ (obtained by means of a variation with fixed endpoints, and up to a reparametrization) if and only if there is no conjugate point to $p$ along $\gamma$.

(3) If a timelike (resp. smooth) curve $\gamma:[a, b] \rightarrow M$ maximizes (resp. minimizes) the time-separation (resp. distance) $d$ then, up to a reparametrization, it is a geodesic without conjugate points except at most $p=\gamma(a)$ and $q=\gamma(b)$.

(4) In a globally hyperbolic spacetime (resp. a complete Riemannian manifold) each two chronologically related points (resp. each two points) can be connected by means of a $d$-maximizing (resp. minimizing) timelike (resp. Riemannian) geodesic. Even more, each inextendible timelike geodesic $\gamma$ : $[0, b) \rightarrow M$ maximizes in some subinterval $[0, c) \subset[0, b), c \in(0, b]$. If $c<b$ then $\gamma(c)$ is called the cut point of $p=\gamma(0)$ along $\gamma$, and cannot appear beyond the first conjugate point.

Moreover, such results can be extended in the Lorentzian case to timelike homotopy classes (i.e., classes of homotopy where the longitudinal curves are timelike). So, fixing $p, q \in M$, if the spacetime is globally hyperbolic 
(resp. the Riemannian manifold is complete) then, in each timelike homotopy (resp. homotopy) class of curves with fixed endpoints $p, q$, there is one connecting timelike (resp. Riemannian) geodesic, with maximum (resp. minimum) length among the curves in that class.

Lightlike geodesics. For lightlike geodesics in Lorentzian manifolds, conjugate points never have accumulation points, too. They cannot appear neither in manifolds of constant curvature nor in dimension 2 (see [63, Proposition 2.34]). The previous four points also hold, with the following modifications:

(1') As in (1), any lightlike geodesic $\gamma$ maximizes $d$ locally. But, now, this means that, for any $p=\gamma(s)$ there exists a neighborhood $U \ni p$ (in fact, any convex neighborhood) such that if $q \in U$ lies on $\gamma$ then no other causal curve contained in $U$ connects $p, q$.

(2') This property is analogous to (2) but recall that, when $\gamma$ is timelike then curves close to $\gamma$ are causal (and timelike), too. The analogous property does not hold for lightlike curves. So, a lightlike geodesic $\gamma:[a, b] \rightarrow M$ which connects two non-conjugate points $p, q \in M$ is, up to a reparametrization, the unique causal curve among close causal curves connecting $p, q$, if and only if there is no conjugate point to $p$ along $\gamma$ (see [63, Section 2]).

(3') If a causal curve $\gamma:[a, b] \rightarrow M$ maximizes the time separation $d$ then, up to a reparametrization, it is a geodesic without conjugate points except at most the endpoints, and then $\gamma$ is lightlike if and only if $d(p, q)=0$.

(4') All the assertions in (4) hold just replacing "chronologically related" by "causally related", and "timelike" geodesics or homotopy class by "causal" one. Nevertheless, causal homotopy classes have remarkable specific properties. For example (see 62 for a detailed study):

Let $\gamma:[0, b) \rightarrow M$ be a lightlike geodesic with a cut point $\gamma\left(\lambda_{c}\right), \lambda_{c} \in(0, b)$. If $\gamma\left(\lambda_{c}\right)$ is not a conjugate point, then:

(1) no other lightlike geodesic which connects $\gamma(0)$ and $\gamma\left(\lambda_{c}\right)$ is causally homotopic to $\gamma$;

(2) if $(M, g)$ is globally hyperbolic, there exists at least another lightlike geodesic $\hat{\gamma}$ (necessarily non-causally homotopic to $\gamma$ ) which connects $\gamma(0)$ and $\gamma\left(\lambda_{c}\right)$.

(For more properties of the cut locus see [11] Chapter 9]; Morse theory for lightlike geodesics was introduced by Uhlenbeck 95 ).

Spacelike geodesics. For spacelike geodesics in Lorentzian manifolds (of dimension $n_{0} \geq 3$ ), as well as for non co-spacelike geodesics in any semi-Riemannian manifold (of index $s_{0}<n_{0}-1$ ), no maximization nor minimization property hold, even though such geodesics are still critical points of the action functional (1.1).

Furthermore, differently from the Riemannian case and the causal geodesics in 
a Lorentzian spacetime, conjugate points along a spacelike geodesic may present accumulation points and even cover a compact interval, so the Morse Index Theorems as in [11, Section 10] cannot be used. In fact, in [54 Section 11] the author constructs a spacelike geodesic in a Lorentzian spacetime which has a continuum of conjugate points while, later on, in [75] Theorem 3.4] the authors prove that "taken any compact subset $F \subset] a, b]$ there exists a 3-dimensional Lorentzian manifold $(M, g)$ and a spacelike geodesic $\gamma:[a, b] \rightarrow M$ such that $\gamma(t)$ is conjugate to $\gamma(a)$ along $\gamma$ if and only if $t \in F$." Anyway, suitable index theories can be developed also in this setting so to prove Morse-type theorems applicable to the spacelike case (see [54, 74] or also [73] for an index theory in more general semi-Riemannian manifolds).

2.3. Conformal changes. For any indefinite semi-Riemannian manifold, the lightlike vectors determine the conformal class of the metric (this is a consequence of a simple algebraic result, see [63. Proposition 2.6]). Thus, two indefinite semi-Riemannian metrics $g, g^{*}$ on $M$ are pointwise conformal, i.e., $g^{*}=\Omega g$ for some (non-vanishing) smooth function $\Omega \in C^{\infty}(M)$, if and only if they have equal lightlike vectors. In what follows, we will asume:

$$
g^{*}=\Omega g, \quad \text { with } \quad \Omega>0, \quad \Omega=e^{2 u}, \quad u \in C^{\infty}(M) .
$$

A straightforward computation from Koszul formula yields the relation between the corresponding Levi-Civita connections $\nabla, \nabla^{*}$ :

$$
\nabla_{X}^{*} Y=\nabla_{X} Y+X(u) Y+Y(u) X-g(X, Y) \nabla u,
$$

where $\nabla u$ denotes the $g$-gradient of $u$, and the equality holds for any vector fields $X, Y \in \mathfrak{X}(M)$. Consequently, one obtains a relation between the corresponding covariant derivatives $D / d t, D^{*} / d t$ and the following equality between the accelerations of any curve $\gamma: I \subset \mathbb{R} \rightarrow M$ :

$$
\frac{D^{*} \gamma^{\prime}}{d t}=\frac{D \gamma^{\prime}}{d t}+2 d u\left(\gamma^{\prime}(t)\right) \cdot \gamma^{\prime}-g\left(\gamma^{\prime}, \gamma^{\prime}\right) \nabla u
$$

In the particular case that $\gamma$ is a lightlike $g$-geodesic, one has:

$$
\frac{D^{*} \gamma^{\prime}}{d t}=f(\gamma) \gamma^{\prime}, \quad \text { with } \quad f(\gamma) \equiv 2 \frac{d(u \circ \gamma)}{d t} \quad \text { on } I .
$$

That is, $\gamma$ is a pregeodesic (i.e., geodesic up to a reparametrization) for $g^{*}$, and the concrete parametrization as a geodesic can be written as $\tilde{\gamma}: J \rightarrow M$ with

$$
\tilde{\gamma}(s)=\gamma(t(s)), \quad s^{\prime}(t)=s_{0}^{\prime} e^{\int_{t_{0}}^{t} f(\sigma) d \sigma} \equiv C e^{2 u(\gamma(t))}, \quad \text { for all } t \in I .
$$

This relation shows that the set of all the lightlike pregeodesics is a conformal invariant. The result can be sharpened, by showing that also their conjugate points and multiplicities are conformally invariant (see [63, Theorem 2.36]). But now, as a simple consequence of the possible maximal domains of definition $I, J$, we have the following completeness result. 
Theorem 2.2. Let $g, g^{*}=\Omega g, \Omega=e^{2 u}$, two indefinite semi-Riemannian metrics on the same manifold $M$. Let $\gamma: I \subset \mathbb{R} \rightarrow M$ be an inextendible lighlike geodesic for $g$, and $\tilde{\gamma}: J \subset \mathbb{R} \rightarrow M$ a reparameterization as an inextendible geodesic of $g^{*}$.

(1) If $\inf (\Omega)>0$ and $\gamma$ is complete $(I=\mathbb{R})$, then $\tilde{\gamma}$ is complete.

(2) If $\sup (\Omega)<0$ and $\gamma$ is incomplete, then $\tilde{\gamma}$ is incomplete.

In particular, if $M$ is compact then $g$ is lightlike complete if and only if so it is $g^{*}$.

Proof. By using the explicit reparametrization (2.2), one has:

Case (1) As $u$ is also lower bounded, $|d s / d t| \geq \epsilon>0$ for some $\epsilon>0$. As $I=\mathbb{R}$ the image $J$ of $s(t)$ also covers all $\mathbb{R}$.

Case (2) Analogously, $(0<) d s / d t<N$, for some $N>0$. Thus, if, say $I=(a, b)$ with $b<+\infty$, then the image of $s(t)$ cannot reach the value $s\left(t_{0}\right)+N\left(b-t_{0}\right)$.

\section{An overview on geodesics in different ambient manifolds and geodesic completeness}

3.1. First results. Our main goals here are: (a) to show an example of independence of causal completitudes, (b) to prove dependence in the locally symmetric case (see Theorem 3.3), (c) to give a criterion on completeness, as an alternative way to Hopf-Rinow Theorem (see Proposition 3.4), and (d) to pose the problem of independence of completeness in the compact case (see Remark 3.7).

Independence of completeness. In general, for an indefinite manifold, the term "completeness" means just geodesic completeness, as there is no any distance canonically associated to the metric. Nevertheless, as there exist spacelike, lightlike and timelike geodesics, one can speak on spacelike, lightlike and timelike completeness, depending on the type of geodesics which are complete. There are explicit examples by Kundt, Geroch and Beem which show the full logical independence of the three types of completeness (see [11 pp. 203] for detailed references). And, in fact, it is easy to construct an example spacelike incomplete and both, timelike and lightlike complete (see Theorem 3.40 for others).

Example 3.1. Concretely, consider in $\mathbb{R}^{2}$ the Lorentz metric

$$
g^{*}=e^{2 u(x, y)}\left(d x^{2}-d y^{2}\right)
$$

conformal to the usual $g_{0}=d x^{2}-d y^{2}$, where $u: \mathbb{R}^{2} \rightarrow \mathbb{R}$ satisfies:

(i) $\partial_{y} u(x, 0)=0$ (in particular $u$ can by chosen $x$-axis symmetric);

(ii) it vanishes outside the horizontal strip $S=\left\{(x, y) \in \mathbb{R}^{2}:|y|<1\right\}$;

(iii) $\int_{-\infty}^{+\infty} e^{u(x, 0)} d x<+\infty$ 
Now, taking into account formula (2.1), one checks easily that the natural reparametrization of the $x$-axis is a $g_{0}$-pregeodesic (use condition (i)), and it is incomplete when parametrized as a (spacelike) geodesic according to (2.2), by using (iii). Nevertheless, any causal geodesic $\gamma$ is complete; in fact, if $\gamma\left(s_{0}\right)$ belongs to the strip $S, \gamma$ will leave $S$ because it cannot remain imprisoned in the compact subset $J^{+}\left(\gamma\left(s_{0}\right)\right) \cap S$ (see in this proceedings 63 Section 3.6.2] for the notions of imprisoned and partially imprisoned curve); this is also easy to check directly because $\gamma$ can be reparametrized as a curve $y \mapsto(x(y), y)$ with $|d x / d y| \leq 1)$ and, outside of $S, \gamma$ is a geodesic of $\mathbb{L}^{2}$.

Remark 3.2. (1) Completeness in any of the three causal types implies inextendibility. That is, if a semi-Riemannian manifold $(M, g)$ is extendible, i.e., it is isometric to an open subset included strictly in another (connected) semiRiemannian manifold $(\tilde{M}, \tilde{g})$, then it is incomplete in the three causal senses, spacelike, lightlike and timelike. In fact, recall that any point in the boundary $\tilde{p} \in \partial M$ can be joined with a point $p$ of $M$ by means of a broken geodesic $\gamma$ of any causal type ${ }^{2}$. Looking $\gamma$ as a curve starting at $p$, each geodesic piece will be included in $M$, i.e., we have the contradiction $\tilde{p} \in M$.

(2) As far as we know, there are no explicit examples which show some independence of any of the three causal type of completeness for compact $M$ (see also Remarks 3.7 and 3.5 (2) below). Nevertheless, Carrière and Rozoy [32] have suggested that one such example will exist in a torus (see Remark 3.27 below).

Dependence in locally symmetric spaces. Recall that in a symmetric semiRiemannian manifold $(M, g)$ there exists a global symmetry at each $p \in M$ (an isometry $I: M \rightarrow M$ which fixes $p$ with $d I_{p}=-\mathrm{Id}$, where Id is the identity at $\left.T_{p} M\right)$ and in the locally symmetric case this symmetry can be found only in a neighborhood of each $p$. Locally symmetric manifolds can be characterized as those with a parallel curvature tensor and, thus, they include constant curvature ones.

The following result is due to Lafuente [58 (point (2)) and Furness and Arrowsmith [45] (point $(3))^{3}$.

Theorem 3.3. (1) A symmetric semi-Riemannian manifold is complete.

(2) In a locally symmetric manifold there is a full dependence among the three types of causal completeness, i.e., completeness in a causal sense implies completeness in the three senses.

(3) Any compact simply connected locally symmetric manifold is complete.

Proof. (1) If $\gamma:[0, b) \rightarrow M$ were a geodesic inextendible to $b<+\infty$, a contradiction is obtained by using the global symmetry at $p=\gamma(2 b / 3)$.

\footnotetext{
${ }^{2}$ This property is needed only for some $p$ (thus, one can choose $p$ in a convex neighborhood of $\tilde{p}$ in $\tilde{M}$ ) but it holds for any $p \in M$.

${ }^{3}$ Elementary results extending naturally the Riemannian ones, such as "a semi-Riemannian manifold is complete if and only if so it is its universal covering", or more refined versions such as 67 Corollary 7.29], will be used without further mention.
} 
(2) The following extension to the semi-Riemannian case, of classical Cartan's result is well-known (see [67, Theorem 8.17]): if $M, \tilde{M}$ are complete connected locally symmetric manifolds, $\tilde{M}$ is simply connected and $L: T_{\tilde{p}} \tilde{M} \rightarrow T_{p} M$ is a linear isometry which preserves the curvature, then there exists a unique semiRiemannian covering map $\phi: \tilde{M} \rightarrow M$ such that $d \phi_{\tilde{p}}=L$. In the proof of this result, one construct the covering map by chaining local isometries along broken geodesics. One can check that only broken geodesics of a causal type are necessary (see [58]) and, thus, the result follows if "completeness" is weakened in "completeness in one causal sense". Thus, if $M$ is complete in a causal sense, its universal covering $\tilde{M}$ will be symmetric (the isometry $\phi: \tilde{M} \rightarrow \tilde{M}$ obtained for $L=-$ Id will be a global symmetry) and, by (1), complete.

(3) Any simply connected symmetric space $M$ can be affinely inmersed in an affine symmetric space of the same dimension and, moreover, when $M$ is compact, the affine inmersion becomes a diffeomorphism (see [45]). Then, the result is a consequence of the proof of (1).

Alternative to Hopf-Rinow: a criterion on completeness. In the case $(M, g)$ is Riemannian, classical Hopf-Rinow Theorem ensures the equivalence between: (a) geodesic completeness, (b) completeness of the associated distance $d_{g}$, and (c) the property that the closed and $d_{g}$-bounded subsets of $M$ are compact. Obviously, in an indefinite manifold (b) does not makes sense, but one could still wonder if the compactness of $M$ would imply completeness. It is well-known that the answer is negative (see below), but we will reasoning now where a proof (which is not based directly on the properties of $d_{g}$ ) would fail; indeed, this will suggest some alternatives for the indefinite case.

Assume that $M$ is compact and $g$ semi-Riemannian, take a geodesic $\gamma:[0, b) \rightarrow$ $M$ with $b<+\infty$, and try to extend it beyond $b$. Recall: (i) given any sequence $s_{n} \nearrow b,\left(\gamma\left(s_{n}\right)\right)_{n}$ converges to some $p \in M$, up to a subsequence, (ii) $\gamma^{\prime}$ can be seen as an integral curve in the tangent manifold $T M$ of the geodesic vector field $G$ on $T M$, (iii) in the Riemannian case, the constancy of $c \equiv\left|\gamma^{\prime}\right|$ also implies that, up to a subsequence, $\left(\gamma^{\prime}\left(s_{n}\right)\right)_{n}$ converges to some $p \in M$ (the bundle of spheres of radius $c$ is compact). Now, recall that, by a well-known result (for example, 67 Lemma 1.56]), if an integral curve $\rho:[0, b) \rightarrow M^{\prime}$ of a vector field $X \in \mathfrak{X}\left(M^{\prime}\right)$ is so that $\left(\rho\left(s_{n}\right)\right)_{n}$ is convergent in $M^{\prime}$, then $\rho$ is extendible beyond $b$. Thus, the extendability in the Riemannian case follows putting $M^{\prime}=T M, X=G, \rho=\gamma^{\prime}$.

Recall that, the crucial step where the positive definite character of the metric (as well as the compactness of $M$ ) are used, is (iii). Thus, previous proof yields directly the following criterion.

Proposition 3.4. Let $(M, g)$ be a semi-Riemannian manifold and $\gamma:[0, b) \rightarrow M$, $b<+\infty$, a geodesic. The following are equivalent:

(i) $\gamma$ is extendible beyond $b$;

(ii) for some (and then for any) complete Riemannian metric $g_{R}$ on $M,\left|\gamma^{\prime}\right|_{R}=$ $g_{R}\left(\gamma^{\prime}, \gamma^{\prime}\right)^{1 / 2}$ is bounded; 
(iii) there exists a sequence $s_{n} \nearrow b$ such that $\left(\gamma^{\prime}\left(s_{n}\right)\right)_{n}$ converges in $T M$.

Remark 3.5. As a consequence, if $\gamma$ is an incomplete geodesic then $\gamma^{\prime}$ cannot be contained in a compact subset of $T M$. Nevertheless, even if $M$ is compact, a complete geodesic may have $\gamma^{\prime}$ not contained in a compact subset (see Remark [3.43(2)).

Limits of incomplete geodesics. Assume in Proposition 3.4 that $\gamma$ is incomplete and $M$ is compact and, thus, $\left|\gamma^{\prime}\left(s_{n}\right)\right|_{R} \rightarrow+\infty$. The set of all the (oriented) directions of $T M$ can be regarded as the $g_{R}$-unit sphere bundle $S_{R} M \subset T M$. If $M$ is compact, then so it is $S_{R} M$ and, therefore, the sequence of directions $v_{n}=\gamma^{\prime}\left(s_{n}\right) /\left|\gamma^{\prime}\left(s_{n}\right)\right|_{R}$ converges to some $v \in S_{R} M$, up to a subsequence. Necessarily, $v$ is lightlike:

$$
g(v, v)=\lim _{n \rightarrow+\infty} g\left(v_{n}, v_{n}\right)=\lim _{n \rightarrow+\infty} c /\left|\gamma^{\prime}\left(s_{n}\right)\right|_{R}=0,
$$

where $c=g\left(\gamma^{\prime}, \gamma^{\prime}\right) \in \mathbb{R}$. Thus, summing up:

Proposition 3.6. Let $(M, g)$ be an incomplete compact semi-Riemannian manifold and $\mathcal{I} \subset S_{R} M$ the set of incomplete directions (i.e., any geodesic with initial velocity in one such direction is incomplete). Then, the closure of $\mathcal{I}$ contains lightlike directions.

Remark 3.7. If $\mathcal{I}$ were closed then incompleteness for compact $M$ would imply lightlike incompleteness. In the non-compact case, it is trivial to show that, in general, $\mathcal{I}$ is neither closed nor open (removing some points of $\mathbb{R}^{2}$ suffices, see [77]). In the compact one, the question has been somewhat controversial, because the closedness of $\mathcal{I}$ had been implicitly assumed in 99. Nevertheless, explicit counterexamples which show that $\mathcal{I}$ is not necessarily closed (nor open) were constructed in [77. (see Remark 3.18 (2) below). Remarkably, in these counterexamples, the limit of some incomplete timelike and spacelike directions is a complete lightlike direction, but there are other incomplete lightlike directions. This lead Romero and Sánchez [7] to pose the independence of incompleteness in the compact case as an open question.

3.2. Completeness under conformal symmetries. Here, we will prove how our criterion of completeness (see Proposition 3.4) can be applied to manifolds with timelike conformal symmetries (see Theorem [3.10), as well as conformally homogeneous manifolds (see Theorem 3.12), obtaining then a generalization of a result by Marsden (see Remark 3.13). Recall that a vector field $K \in \mathfrak{X}(M)$ is called conformal Killing if the Lie derivative $\mathcal{L}$ satisfies

$$
\mathcal{L}_{K} g=2 \sigma g
$$

(the local flows of $K$ are conformal maps) for some $\sigma \in C^{\infty}(M)$. If $\gamma$ is a geodesic:

$$
\frac{d}{d s} g\left(\gamma^{\prime}, K\right)=c \sigma \circ \gamma, \text { for } c=g\left(\gamma^{\prime}, \gamma^{\prime}\right)
$$

In the case $\sigma \equiv 0, K$ is Killing and $g\left(\gamma^{\prime}, K\right)$ is a constant. 
General technique. As a first application of Proposition 3.4 for the Lorentzian case (and using the notation there in what follows) we have the following result by Romero and Sánchez [80]:

Proposition 3.8. Let $(M, g)$ be a compact Lorentzian manifold. If it admits a timelike conformal Killing vector field then $g$ is complete.

Proof. Because of Lorentzian signature, the orthogonal bundle $K^{\perp}$ is Riemannian and, as $g\left(\gamma^{\prime}, \gamma^{\prime}\right)$ is constant, it is enough to check that the projection of $\gamma^{\prime}$ in $\operatorname{span}(K)$ lies in a compact subset. But this follows directly, because (3.1) implies that $g\left(\gamma^{\prime}, K\right)$ is bounded on $[0, b)$, if $b<+\infty$.

Remark 3.9. The result does not hold if $K$ is allowed to be causal (in particular, non-vanishing) at some points (see Remark 3.18).

Obviously, previous result can be extended to the case of index $s_{0}$ if there are $s_{0}$ pointwise-independent Killing vector fields. Even more, the compactness assumption can be dropped if some additional conditions are imposed, yielding the following general result (see [79]):

Theorem 3.10. A semi-Riemannian manifold $(M, g)$ of index $s_{0}$ is complete if there exist $s_{0}$ timelike conformal-Killing vector fields $\left\{K_{1}, \ldots K_{s_{0}}\right\}$ satisfying:

(i) the Gram matrix $\left\{g\left(K_{i}, K_{j}\right)\right\}$ has inverse $g^{i j}$, and $\sum_{i, j}\left(g^{i j}\right)^{2}$ is bounded;

(ii) functions $\sigma_{i}$ satisfying (3.1) for the corresponding $K_{i}$, are bounded;

(iii) the associated Riemannian metric $g_{R}$ is complete, where $g_{R}$ is given by

$$
g_{R}(X, Y)=g(X, Y), \quad g_{R}(A, B)=-g(A, B), \quad g_{R}(X, A)=0
$$

for any $A, B \in \operatorname{span}\left\{K_{1}, \ldots, K_{s_{0}}\right\}$ and $X, Y$ belonging to its $g$-orthogonal complement.

Remark 3.11. An example of metric where this result is applicable is provided by warped fiber bundles, type Kaluza-Klein as the following. Let $P(B, G), \pi_{B}$ : $P \rightarrow B$, a principal bundle on the complete Riemannian manifold $\left(B, g_{B}\right)$ with structural group $G$, and let $\omega: P \rightarrow \mathcal{G}$ be a connection 1-form on the Lie algebra $\mathcal{G}$ of $G$. For any positive function $f: B \rightarrow \mathbb{R}$ whose infimum satisfies inf $f(B)>0$, and any bi-invariant metric $g_{G}$ on $\mathcal{G}$ (if it is semi-simple, its Killing form suffices), define $g=\pi_{B}^{*} g_{B}+f^{2} \omega^{*} g_{G}$. The fundamental vectors of the bundle yields enough Killing vector fields to ensure completeness.

Homogeneous manifolds. Previous technique also works for (conformally) homogeneous manifolds. Recall that in a homogeneous manifold $M$ any point $p \in M$ can be mapped to another one $q \in M$ by means of an isometry. Remarkably then, any tangent vector $v \in T_{p} M$ can be extended to a Killing vector $V$ (for a conformal metric, $V$ will be Killing conformal). 
Theorem 3.12. A compact semi-Riemannian manifold which is globally conformal to a homogeneous semi-Riemannian manifold is complete.

Proof. In order to apply Proposition 3.4 take $p=\lim _{n \rightarrow+\infty} \gamma\left(t_{n}\right)$, extend a basis of $T_{p} M$ to a set of conformal Killing vector fields (which will be a pointwise basis in a neighborhood of $p$ ) and use (3.1).

Remark 3.13. (1) This is an extension of a result by Marsden [59], who considered the homogeneous case. In this case $T M$ can be divided in compact subsets which are invariant by the geodesic flow. Nevertheless, this property may not hold in the conformal case (see Remarks 3.5 and 3.43(2)).

(2) It is well-known that a homogeneous Riemannian manifold is complete (use that at one point $p \in M$ the closed ball of some radius $r>0$ is compact; by homogeneity, this happens with the same $r$ at any $q \in M$, and this property yields completeness easily). This does not hold in the indefinite case (see below) and, in fact, there are even incomplete compact locally homogeneous manifolds (see [52]).

3.3. Heuristic construction of incomplete examples. Now, our purpose is to construct examples of compact incomplete semi-Riemannian manifolds. Even though one such example can be exhibited directly, we will construct it in a heuristic way, and will find typical related items along the construction: incomplete homogeneous manifolds, incomplete closed lightlike geodesics, Misner's cylinder...

Incomplete homogeneous manifolds. Let us start with Lorentz-Minkowski spacetime $\mathbb{L}^{2}=\left(\mathbb{R}^{2}, g_{0}\right)$, with its metric expressed in lightlike coordinates, and a semiplane $M_{0}$ :

$$
g_{0}=d u \otimes d v+d v \otimes d u, \quad M_{0}=\left\{(u, v) \in \mathbb{L}^{2}: u>0\right\} .
$$

Obviously, for any $\mu \neq 0$, the map

$$
\phi(u, v)=(\mu u, v / \mu)
$$

is an isometry of $\mathbb{L}^{2}$ inducible in $M_{0}$. As so they are the translations in the direction of the $v$ axis too, $\left(M_{0}, g_{0}\right)$ is an incomplete homogeneus semi-Riemannian manifold.

Quotients by isometries and Misner's cylinder. Now, consider the isometry group $G$ of both $\mathbb{L}^{2}$ and $M_{0}$, generated by $\phi \equiv \phi_{2}$, i.e., $\phi(u, v)=(2 u, v / 2)$. Consider first the action of $G$ on $\mathbb{L}^{2}$. As the origen is a fixed point, the action of $G$ is not free. Nevertheless, it yields a free and discontinuous action ${ }^{4}$ on $\mathbb{R}_{*}^{2}=\mathbb{R}^{2} \backslash\{(0,0)\}$.

\footnotetext{
${ }^{4}$ An action - of a group $G$ on a topological space $X$ is free if the equality $g \cdot x=x$ for some $x \in X, g \in G$ implies that $g$ is the the unit element $e$ of $G$. It is discontinuous if, for any sequence $\left(g_{m}\right)_{m}$ of distinct elements of $G$ and $x \in X$, the sequence $\left(g_{m} \cdot x\right)_{m}$ is not convergent. The requirements for a properly discontinuous action are: (i) if $x, x^{\prime} \in X$ do not lie in an orbit by $G$ $\left(x^{\prime} \neq g \cdot x\right.$ for all $\left.g \in G\right)$ there are neighborhoods $U \ni x, U^{\prime} \ni x^{\prime}$ such that $g U \cap U^{\prime}=\emptyset$ for all $g \in G$, (ii) the isotropy group $G_{x}$ at each $x \in X\left(G_{x}=\{g \in G: g \cdot x=x\}\right)$ is finite, and (iii) for all $x \in X$, there exists an open neighborhood $U \ni x$ such that: $g U \cap U=\emptyset$ for all $g \in G \backslash G_{x}$.
} 
In the Riemannian case, these two conditions for an isometry action are sufficient to obtain a (Hausdorff) quotient manifold, as the action will be properly discontinuous (see [57. I, Chapter 1], especially Proposition 4.4). Nevertheless, this is not enough in the indefinite case and, in fact, the quotient $\mathbb{R}_{*}^{2} / G$ is non-Hausdorff.

Remark 3.14. Let $\mathcal{M}_{n}$ be the group of rigid motions of $\mathbb{R}^{n}$ (semidirect product of translations and rotations) and $\mathcal{A}_{n}$ the one of affine maps (idem with translations and linear automorphisms). As a consequence of previous discussion, if a subgroup $G<\mathcal{M}_{n}$ acts freely and discontinuously on $\mathbb{R}^{n}$ the quotient is a (Hausdorff) manifold. Nevertheless, the same assertion for $G<\mathcal{A}_{n}$ was pointed out as open by Charlap in 34 pp. 4].

The action of $G$ on $M_{0}$ in (3.2) is properly discontinuous and, in fact, the quotient $C_{0}=M_{0} / G$ is topologically a cylinder (see $C_{0}$ as $\left\{(u, v) \in \mathbb{L}^{2}: 1 \leq u \leq 2\right\}$ with each $(1, v)$ identified to $(2, v / 2))$, endowed with a Lorentzian metric. This Lorentzian manifold (a simplified example for the remarkable geometric properties of Taub-NUT spacetime 64) will be called Misner's cylinder. Obviously, $C_{0}$ is incomplete in the three causal senses, as so it is $M_{0}$. More strikingly, $M_{0}$ contains a closed incomplete lightlike geodesic, concretely the projection $\gamma_{0}$ of the $M_{0}-$ geodesic $\tilde{\gamma}_{0}(s)=(-s, 0)$ for all $s<0$.

Closed incomplete geodesics. The reason for the incompleteness of $\gamma_{0}$ can be explained as follows. Choose $s_{1}<0$, put $s_{2}=s_{1} / 2, T=s_{2}-s_{1}>0$ and recall that, from $s_{1}$ to $s_{2}$ the geodesic gives a round $\left(\gamma_{0}\left(s_{1}\right)=\gamma_{0}\left(s_{2}\right)\right)$, but the velocity after this round satisfies $\gamma^{\prime}\left(s_{1}\right)=2 \gamma\left(s_{0}\right)$. Thus, $\gamma_{0}$ will spend a time $T / 2$ in giving a second round and, in general, $T / 2^{k}$ for $k$ new rounds. This yields directly its incompleteness. As a straightforward generalization we have.

Definition 3.15. Let $(M, g)$ be a semi-Riemannian manifold, $\gamma: I=(a, b) \rightarrow M$ a (inextensible) non-constant geodesic. Then, $\gamma$ is closed if there exists $\lambda>0$ and $s_{1}, s_{2} \in I, s_{1}<s_{2}$ such that $\gamma(a)=\gamma(b)$ and:

$$
\gamma^{\prime}\left(s_{1}\right)=\lambda \gamma^{\prime}\left(s_{2}\right)
$$

In the case, if $\lambda=1$ then $\gamma$ is called periodic.

If equality (3.3) holds for some non-constant geodesic $\gamma$ then:

(i) Necessarily, $\lambda>0$. In fact, $\lambda \neq-1$ because, in this case, the standard uniqueness of geodesics yields $\epsilon>0$ such that $\gamma\left(s_{1}+s\right)=\gamma\left(s_{2}-s\right)$ for all $s \in[0, \epsilon]$. Even more, the points which satisfy this equality form an open and closed subset of $\left[s_{1}, s_{2}\right]$, obtaining the contradiction $\gamma^{\prime}\left(s_{c}\right)=-\gamma^{\prime}\left(s_{c}\right)$ at $s_{c}=\left(s_{2}-s_{1}\right) / 2$. A similar contradiction happens if $\lambda<0$.

(ii) If $\lambda \neq 1$ then necessarily $\gamma$ is lightlike (otherwise, the constancy of $g\left(\gamma^{\prime}, \gamma^{\prime}\right.$ ) yields a contradiction) and, by the reasoning above, incomplete. Thus, the notions closed and periodic are interchangeable for spacelike and timelike geodesics.

(iii) If $\gamma$ is periodic then there exists a minimum $T>0$ (the period) such that $\gamma(t)=\gamma(t+T)$, for all $t$. In particular, $\gamma$ is complete.

Summing up: 
Proposition 3.16. Let $\gamma: I \rightarrow M$ be a closed geodesic in $(M, g)$.

(1) If $\gamma$ is not lightlike then it is periodic.

(2) $\gamma$ is complete if and only if it is periodic. In fact, if (3.3) holds with $\lambda>1$ (resp. $\lambda<1)$ then $I=(-\infty, b), b<+\infty$ (resp. $I=(a,+\infty), a>-\infty)$.

Construction of incomplete compact manifolds. It is not difficult to realize that the metric of Misner's cylinder $\left(C_{0}, g_{0}\right)$ in Subsection 3.3 can be modified outside a strip $|v| \leq R$ in order to obtain a new Lorentzian metric which can be induced in a torus by identifiying the points with $v=2 R$. A way to make this explicitly is the following (see [77, 81]).

Consider the global change of variables $\Psi: \mathbb{R}^{2} \rightarrow M_{0}=\mathbb{R}^{+} \times \mathbb{R}, u(x, y)=e^{y}$, $v(x, y)=x e^{-y}$ and the pull-back

$$
g_{\tau}=\Phi^{*} g_{0}=d x \otimes d y+d y \otimes d x+\tau(x) d y^{2}
$$

where $\tau(x)=-2 x$. The generator $\phi$ of Misner's group $G$ corresponds to the translation $(x, y) \mapsto(x, y+\log 2)$, and the incomplete geodesic $\tilde{\gamma}$ of $M_{0}$ to a reparametrization of the axis $x \equiv 0$. So, if we choose as $\tau(x)$ a periodic function which behaves as $-2 x$ around $x=0$, an incomplete metric on $\mathbb{R}^{2}$ is obtained. This can be induced in a quotient torus obtained by means of two translations in the directions of the two axis. Even more, by an explicit computation of the geodesics of $g_{\tau}$ (which can be easily integrated as $g_{\tau}\left(\gamma^{\prime}, \gamma^{\prime}\right)$ and $g_{\tau}\left(\gamma^{\prime}, \partial_{y}\right)$ are constants) it follows:

Proposition 3.17. If $\tau(0)=0$ but $\tau \not \equiv 0$, then metric $g_{\tau}$ in 3.4 contains timelike, spacelike and lightlike incomplete geodesics (which asymptote the axis $x \equiv 0$ ).

Thus, if $\tau$ is additionally 1-periodic, the quotient torus $T^{2}=\mathbb{R}^{2} / Z^{2}$, with the induced metric from $g_{\tau}$, is incomplete in the three causal senses.

Remark 3.18. Under the stated hypotheses for $\left(T^{2}, g_{\tau}\right)$ :

(1) If we choose $\tau \leq 0$ then the vector field induced in $T^{2}$ from $K=\partial_{y}$ becomes Killing and causal, showing that Proposition 3.8 cannot be extended to this case.

(2) If $\tau^{\prime}(0)=0$ then the axis $x \equiv 0$ can be reparametrized as a complete geodesic, showing that the incomplete directions $\mathcal{I}$ (see Proposition 3.6) are not closed.

(3) On the other hand, if one chooses either $\tau>0$ or $\tau<0$, the torus will be complete (this can be computed directly or deduced as a consequence of Theorem 3.23 below).

Remark 3.19. Starting by one such incomplete torus, it is not difficult to find another interesting incomplete semi-Riemannian manifolds. For example (see [52]), one can construct an incomplete solid torus and, taking into account that the 3-sphere can be obtained by gluing two solid tori, a simply connected compact incomplete Lorentzian manifold can be constructed. 
3.4. Surfaces. We have already seen quite a few bidimensional examples, which become interesting for both, simplicity and to test possible results (see [92]). Here, we collect the properties of any such a Lorentzian surface $(S, g)$, focusing in the cases $S=\mathbb{R}^{2}, S=T^{2}$ (as the Euler characteristic of $S$ would be 0 , see 63] Theorem 2.4], the former is the unique simply connected case and the latter the unique compact orientable case). Our main goals are to study: (a) for $S=\mathbb{R}^{2}$, the stability of completeness in the $C^{r}$ topologies (see Theorem 3.20), (b) conformally flat tori, including stability of completeness (see Theorem 3.23), (c) structure of tori with a Killing field, including the dependence of causal completitudes (see Theorem 3.24), and (d) general tori, including the problem of dependence of completitudes, studied by means of its two lightlike foliations. Recall that any Lorentzian surface admits lightlike coordinates and, thus, it is locally conformally flat (see, for example, 98]). So, conformally flat means globally conformal to a flat manifold.

Case $S=\mathbb{R}^{2}$. A remarkable difference with the Riemannian case appears for the conformal class of $\left(\mathbb{R}^{2}, g\right)$. If $g$ is Riemannian, Theorem of Uniformization implies that $g$ is conformally equivalent either to the unit disc or to $\mathbb{R}^{2}$ with their natural metrics. Nevertheless, if $g$ is Lorentzian there exist infinitely many conformal classes (see Weinstein [98] for a detailed study or [63, footnote 10] in these proceedings).

Any metric $g$ on $\mathbb{R}^{2}$ is stably causal (see 63, Theorem 3.55]) and, as $-g$ is also Lorentzian, the roles of spacelike and timelike geodesics are interchangeable. In particular, no inextendible geodesic of any causal type is imprisoned in a compact subset in the forward or backward direction. Thus, the results of stability of geodesic incompleteness in [11. Theorem 7.30], or Theorem 3.52 below (we also refer to [1] or 63] for definitions) apply, yielding:

Theorem 3.20. If the Lorentzian surface $\left(\mathbb{R}^{2}, g\right)$ is incomplete in some causal sense, then there is a $C^{1}$-neighborhood $U(g)$ of $g$ such that each metric $g_{1}$ in $U(g)$ is incomplete in that causal sense.

Remark 3.21. The $C^{1}$ stability of the completeness of surfaces as $\mathbb{L}^{2}$ is also known (see Theorem 3.54 below).

Case $S=T^{2}$. Recall first the following result.

Lemma 3.22. (1) Any flat Lorentzian torus is complete and, in fact, the quotient of $\mathbb{L}^{2}$ by the action generated by two independent translations.

(2) Infinitely many classes of non-conformally related flat Lorentzian torus exist.

(3) The torus which admit a periodic lightlike geodesic $\gamma_{0}$ are dense in the set of all the flat Lorentzian torus, for any $C^{r}$-topology.

Proof. (1) Completeness (and, then, the structure result) can be seen as a particular case of Theorem 3.30 below, but direct proofs are possible (see [44, 46]).

(2) Choose as generators of the translations $v=(1,0)$ and $w_{k}=(0, k)$ for $k$ a (prime) natural number. Each quotient torus $T_{k}$ admits a periodic lightlike pregeodesic (which is a conformal invariant) in a different free homotopy class. 
(3) This property follows from a commensurability argument between the generators of the translations, using the density of the rationals in $\mathbb{R}$.

Now, we can see that, even though (conformally) flat Lorentzian tori are complete, this is an unstable property (see [78]):

Theorem 3.23. (1) A Lorentzian torus $\left(T^{2}, g\right)$ is conformally flat if and only if it admits a timelike (or spacelike) conformal vector field $K$. Thus, it is complete.

(2) Conformally flat Lorentzian metrics on $T^{2}$ lie in the closure of the set of lightlike incomplete Lorentzian metrics. Moreover, the flat ones lie in the closure of timelike, lightlike and spacelike incomplete Lorentzian metrics.

Proof. (1) $(\Rightarrow$ ) From Lemma 3.22(1), any flat torus admits a parallel vector field of any causal character, which will be conformal Killing for any conformal metric.

$(\Leftarrow)$ Easily, $K$ is Killing for the conformal metric $g^{*}=g /|K|$ and, in dimension 2 , it is also parallel (the last assertion follows from Proposition 3.8).

(2) By Lemma 3.22(3), and the conformal invariance of the lightlike incompleteness (see Theorem [2.2), it is enough to prove the property for the flat tori which admit a periodic lightlike geodesic $\gamma_{0}$. Recall that this geodesic is lifted to the universal covering $\mathbb{R}^{2}$ as an affine parametrization of $x \equiv 0$. Now, choose $\tau$ as in Proposition 3.17 arbitrarily close to 0 in the $C^{r}$ topology.

The case of non-conformally flat Lorentzian tori admitting a Killing vector field $K$ can be also characterized in a precise way (see 84]); surprisingly, all of them are incomplete. In order to describe not only this result but also the structure of these tori, consider first the following generalization of the metric (3.4):

$$
g[(x, y)]=E(x) d x^{2}+F(x)(d x \otimes d y+d y \otimes d x)-G(x) d y^{2},
$$

where $E, F, G \in C^{2}(\mathbb{R})$ satisfy the following conditions: (i) $E G+F^{2}>0$, that is, $g$ is Lorentzian, and (ii) $E, F$ and $G$ are periodic with the same period 1, so, the metric is naturally inducible on a torus $T^{2}=\mathbb{R}^{2} / Z^{2}$. As the vector field $K=\partial / \partial y$ is Killing, if $|G|$ were greater than 0 at every point, then $K$ would be either timelike or spacelike, and thus, $g$ is conformally flat. Moreover, if $G \equiv 0$ then $K$ is lightlike and $g$ is flat. Now, denote by $\mathcal{G}$ (resp. $\mathcal{G}^{c}$ ) the set of metrics given by (3.5) and satisfying (i), (ii) and also: (iii) the sign of $G$ is not constant (resp. (iii) ${ }^{c}$ the function $G$ is not constant and $|G|>0$ ). Recall that the geodesic equations for $\gamma(t)=(x(t), y(t))$ can be explicitly integrated (see Proposition 3.17). This, combined with some technicalities (for example, a non-trivial Killing vector field cannot vanish on a torus), yields (see [84]):

Theorem 3.24. If a Lorentzian torus $\left(T^{2}, g\right)$ admits a Killing vector field $K \not \equiv 0$, then $K$ does not vanish at any point and:

(1) The metric $g$ is flat if and only if $g(K, K)$ is constant.

(2) The metric $g$ is conformally flat if and only if $g(K, K)$ has a definite sign (strictly positive, strictly negative or identically zero), and if and only if $g$ is geodesically complete (in the three causal senses). 
Moreover, $g$ is conformally flat but non-flat if and only if it is, up to a covering, isometric to one of the $\mathcal{G}^{c}$-tori constructed above.

(3) The metric $g$ is non-conformally flat if and only if $g$ is geodesically incomplete in one (and then in the three) causal senses, and if and only if it is, up to a covering, isometric to one of the $\mathcal{G}$-tori constructed above.

Remark 3.25. (1) For tori admitting a Killing vector field there is equivalence between the three types of causal completeness.

(2) For a torus conformal to a previous one, all the assertions in Theorem 3.24 hold with obvious modifications, except that, in principle, one can ensure only the equivalence with lightlike incompleteness in the case (3).

(3) One of these incomplete tori is the celebrated Clifton-Pohl one, usually defined as the quotient of $\left(\mathbb{R}_{*}^{2}, g=\left(u^{2}+v^{2}\right)^{-1}(d u \otimes d v+d v \otimes d u)\right)$ by the isometry group generated by the homothety $(u, v) \mapsto(2 u, 2 v)$ for all $(u, v) \in \mathbb{R}_{*}^{2}$. Necessarily, it is a $\mathcal{G}$-torus $(K=u \partial / \partial u+v \partial / \partial v$ is Killing), but this can be checked directly (see [84, Remark 5.2]).

When no (conformal) Killing on $T^{2}$ exists, the study of the geodesics becomes more difficult. The completeness of geodesics in simultaneously all the elements of a conformal class of Lorentzian tori has been studied from the next point of view by Carrière and Rozoy in 32. Consider the two (conformally invariant) foliations $\mathcal{F}, \mathcal{H}$ yielded by the lightlike geodesics of $T^{2}$. Then one has (we refer to 32] and references therein for the explanation of concepts relative to foliations):

Theorem 3.26. (1) For arbitrary $\mathcal{H}$ :

(1a) if $\left(T^{2}, g\right)$ is lightlike complete then $\mathcal{F}$ and $\mathcal{H}$ are $C^{0}$-linearizable;

(1b) if $\mathcal{F}$ and $\mathcal{H}$ are $C^{1}$-linearizable then $g$ is lightlike complete.

(2) In the particular case that $\mathcal{H}$ is a foliation by circles, then $\mathcal{F}$ is obtained from suspension of a diffeomorphism $\phi$ of the circle, and then:

(2a) $g$ is lightlike-complete if and only if $\phi$ is $C^{0}$-conjugate to a rotation,

(2b) if $\phi$ is $C^{1}$-conjugate to a rotation then $g$ is complete.

Remark 3.27. As a consequence of Theorem [3.26] one has that at least when $\mathcal{H}$ is by circles, "generically" any incomplete Lorentzian tori must be lightlike incomplete (for almost all real number, $C^{0}$-conjugate to a rotation implies $C^{1}$ ). Nevertheless, there are residual cases which are compatible with $g$ incomplete but lightlike complete; this suggests that such a possibility will hold.

3.5. Influence of curvature. In semi-Riemannian Geometry, as in the Riemannian one, the curvature determines the metric (for example, in the sense of Cartan's result, see the proof of Theorem 3.3(2)). Nevertheless, there are very subtle questions for a global property such as completeness, in the indefinite case. Here, we show first that the curvature does not characterize completeness, even in the compact case (see Theorem 3.28). This stresses Markus' conjecture as well as the completeness of compact manifolds of constant curvature (see Theorem 3.301). The nice behaviour of geodesics in the complete case (i.e., spaceforms), 
is described around Theorem 3.32 Finally, even though relativistic Singularity Theorems become a substantial topic in its own right (see 91), we give some flavour of them in comparison with Riemannian Geometry (see Theorems 3.33 3.34).

Complete and incomplete torus with the same curvature. A first strong sense of independence between curvature and completeness is the following (see [77):

Theorem 3.28. There are two metrics $g_{1}, g_{2}$ on the same torus $T^{2}$, with the same curvature at each point and such that the first one $g_{1}$ is incomplete and the second one $g_{2}$ is complete.

Proof. Consider the metric (3.4), which has Gauss curvature $\tau^{\prime \prime} / 2$. Now, choose as $\tau$ for $g_{1}$ any 1 -periodic function $\tau_{1}$ with $\tau_{1}(0)=0 \neq \tau_{1}^{\prime}(0)$ (it is incomplete by Proposition 3.17). For $g_{2}$ put $\tau_{2}=\tau_{1}+N$, where $N$ is chosen such that $\tau_{2}>0$, and notice that it is complete by Remark 3.18(3).

Remark 3.29. This result can be generalized to obtain complete and incomplete Lorentzian torus with prescribed curvature $k=k(x)$, satisfying the condition of compatibility with Gauss-Bonnet Theorem (i.e., $\int_{0}^{1} k(x) d x=0$ ). Applications to solutions of D'Alembert equation can be seen in 84 .

Markus conjecture. In spite of previous result, one can wonder if a strong restriction on the curvature, as flatness, will imply completeness. Such a question is a particular case of Markus conjecture on affine manifolds.

An affine manifold is a manifold $M$ locally modelled on open subsets of $\mathbb{R}^{n}$ such that the changes of coordinates are elements of the group of affine transformations $\operatorname{Aff}\left(\mathbb{R}^{n}\right)$. Fixing $p \in M$ one has the natural representation of holonomy for the fundamental group $\pi_{1}(M), h: \pi_{1}(M) \mapsto h\left(\pi_{1}(M)\right)=\Gamma \subset \operatorname{Aff}\left(\mathbb{R}^{n}\right)$, which is independent of $p$ up to conjugacy. Let $L(\Gamma) \subset \mathrm{Gl}\left(\mathbb{R}^{n}\right)$ be the linear part of $\Gamma$ obtained from the natural projection.

Conjecture (Markus): An unimodular (i.e., $L(\Gamma) \subset S L\left(\mathbb{R}^{n}\right)$ ) compact affine manifold is complete.

In the flat Lorentz case one has naturally an affine manifold with $L(\Gamma)$ included (up to an orientable covering) in the special Lorentz group $\mathrm{SO}_{1}(n)$. Carrière [31] solved this case by: (i) introducing an invariant for any subgroup $G \subset \operatorname{Gl}\left(\mathbb{R}^{n}\right)$, the discompactness $\operatorname{disc}(G)$, which measures at what extent $G$ fails to be compact (in particular, $\operatorname{disc}(\mathrm{SO}(n))=1$ ), (ii) proving Markus' conjecture for $\operatorname{disc}(\mathrm{SO}(n)) \leq 1$. Even more, Klingler [56] extended this result to include the manifolds of constant curvature. Summing up:

Theorem 3.30. Any compact Lorentzian manifold $(M, g)$ of constant curvature is complete. 
Remark 3.31. (1) As far as we know, the question remains open for $g$ indefinite with higher index.

(2) Obviously, here the techniques are very different to those ones for Proposition 3.4 and, in particular, they are not conformal invariant. Thus, one can wonder if completeness will hold for any conformal metric $g^{*}=\Omega g$. This will hold if $M$ is a $n$-torus or a nilmanifold, because in this case $(M, g)$ will admit a timelike Killing vector field and Proposition 3.8 applies.

(3) Notice that:

$$
\text { constant curvature } \Rightarrow \text { locally symmetric } \Rightarrow \text { locally homogeneus. }
$$

As we have seen, at least in the compact Lorentzian case the first condition implies completeness, but the last one does not (see Remark 3.13). As far as we know, also the intermediate compact locally symmetric case remains open.

Spaceforms. A semi-Riemannian $n$-dimensional manifold $M$ of index $s$ is a spaceform if it is complete with constant curvature. This curvature will be regarded as normalized to $\epsilon=-1,0,1$. Simply connected spaceforms are called model spaces and are characterized by $n, \epsilon, s$. Essentially, the model spaces are either $\mathbb{R}_{s}^{n}$ or the pseudosphere $\mathbb{S}_{s}^{n}$ (spacelike vectors of norm 1 in $\mathbb{R}_{s}^{n+1}$ ); but for $n=2$ the pseudosphere is topologically $\mathbb{S}^{1} \times \mathbb{R}$ and its universal covering must be taken. Any spaceform can be constructed as the quotient of its model space by a group $\Lambda$. As we have seen above, if the assumption of completeness is dropped then the universal covering may be a proper open subset of the model space, which makes the study more difficult.

Geodesics on $\mathbb{S}_{s}^{n}$ can be constructed by intersecting $\mathbb{S}_{s}^{n}$ with a plane in $\mathbb{R}_{s}^{n+1}$ which crosses the origin. Such geodesics are well known (see 67, Proposition 5.38]) and, in particular, no indefinite pseudosphere $\mathbb{S}_{s}^{n}, 0<s<n$ is geodesically connected. Nevertheless, even non-flat spaceforms may be geodesically connected. We recall the following result by Calabi and Markus [19] (which, in particular, solves completely the geodesic connectedness of Lorentzian spaceforms of positive curvature with $n \geq 3$ ) and refer also to [67, 89] for further information.

Theorem 3.32. (1) Two points $p, q \in \mathbb{S}_{1}^{n}(n \geq 2)$ are connectable by a geodesic if and only if $\langle p, q\rangle_{1}>-1$, where $\langle\cdot, \cdot\rangle_{1}$ is the inner product of $\mathbb{L}^{n+1} \equiv \mathbb{R}_{1}^{n+1}$.

(2) Any spaceform $M=\mathbb{S}_{1}^{n} / \Lambda, M \neq \mathbb{S}_{1}^{n}$ is starshaped from some points $p \in M$.

(3) A spaceform $M=\mathbb{S}_{1}^{n} / \Lambda$ is geodesically connected if and only if it is not timeorientable.

The proof of (1) follows from a direct computation of the geodesics. For the remainder, the essential idea is that (whenever $2 s \leq n$ ), the group $\Lambda$ is finite. Then, up to conjugacy, $\Lambda \subset O(1) \times O(n) \subset O_{1}(n+1)$, and the proof follows from studying the barycenter of the orbits, which must lie in the timelike axis of $\mathbb{R}_{1}^{n+1}$.

Singularity theorems. In Riemannian Geometry, it is well-known that negative (sectional) curvature implies divergence of geodesics, while positive curvature, 
or even just positive Ricci curvature, implies convergence and focalization. This result, in combination with properties of the distance function, implies bounds on the diameter (and, then, compactness) for complete Riemannian metrics with Ricci curvature greater than some positive constant (see Myers' Theorem). In Lorentzian manifolds the properties of convergence and focalization hold analogously for timelike geodesics (and, with some particularities, for lightlike ones) under positive Ricci curvature on timelike vectors (or negative sectional curvature on timelike planes) $)^{5}$. Nevertheless, the inexistence of a true distance in a Lorentzian manifold and the particular properties of their global structure, make so that the natural conclusion cannot be the finiteness of the diameter but the causal incompleteness of the manifold.

In fact, in the framework of General Relativity, singularity theorems are incompleteness theorems for causal geodesics. Essentially, there are two types which prove: (1) the existence of incomplete timelike geodesics in a cosmological setting, and (2) the existence of an incomplete lightlike geodesic in the context of gravitational collapse and black holes. In general, they use some refined properties of Causality which lie out of the scope of the present paper. But we can give some flavour of them by studying a "Big Bang" singularity theorem which has a clear correspondence with the Riemannian ideas commented above.

Recall the following Hawking's Singularity Theorem (the necessary concepts on Causality can be seen in these proceedings [63; see [53, 67] or 91] for a detailed exposition of this singularity theorem and others):

Theorem 3.33. Let $(M, g)$ be a spacetime such that:

1. it is globally hyperbolic;

2. some spacelike Cauchy hypersurface $S$ is strictly expanding $H \geq C>0$ ( $H$ is the mean curvature with respect to the future direction);

3. $\operatorname{Ric}(v, v) \geq 0$ for timelike $v$.

Then, any past-directed timelike geodesic $\gamma$ is incomplete.

Proof. The last two hypotheses imply that any past-directed geodesic $\rho$ normal to $S$ contains a focal point if it has length $L^{\prime} \geq \frac{1}{C}$. Thus, once $S$ is crossed, no $\gamma$ can have a point $p$ at length $L>\frac{1}{C}$ (otherwise, a length-maximizing timelike geodesic from $p$ to $S$ with length $L^{\prime} \geq L$ would exist by global hyperbolicity, a contradiction).

Now, as an exercise, the reader can prove the Riemannian result below and stress the isomorphic roles of:

\section{Global hyperbolicity $\longleftrightarrow$ Riemannian completeness}

\footnotetext{
${ }^{5}$ The imposition of inequalities only on timelike vectors (or on timelike planes) becomes essential for the mathematical non-triviality of the problem, as well as for physical interpretations. So, inequality $\operatorname{Ric}(v, v) \geq 0$ for any tangent vector $v$ imply (in dimension $\geq 3$ ) that the manifold is Einstein. But this inequality imposed only for timelike $v$ is mathematically natural, and admits the physical interpretation that gravity, on average, attracts (timelike convergence condition).
} 
2. $\operatorname{Ric}(v, v) \geq 0$ for timelike $v \longleftrightarrow \operatorname{Ric}(v, v) \geq 0$ for all tangent $v$

3. Timelike incompleteness (bounded lengths of timelike geodesics starting at $S) \longleftrightarrow$ Finite Riemannian distance to $S$.

Theorem 3.34. Let $(M, g)$ be a Riemannian manifold such that:

1. it is complete;

2. some closed (as a subset) hypersurface $S$ separates $M$ as the disjoint union $M=M_{-} \cup S \cup M_{+}$, and $S$ is strictly expanding towards $M_{+}: H \leq-C<0$ (with appropiate sign convention for $H$ );

3. $\operatorname{Ric}(v, v) \geq 0$ for all $v$.

Then, $\operatorname{dist}(p, S) \leq 1 / C$ for all $p \in M_{-}$.

3.6. Warped products. Here, we study the behaviour of geodesics in a general warped product. For geodesic completeness, the details of the fiber become irrelevant (see Theorem 3.37), and leads to the notion of warped completeness. This can be characterized very accurately in the case of definite base (see Theorem 3.37), and is related to some open questions in the case of indefinite base (see Remark 3.45).

A warped product $B \times{ }_{f} F$ of the semi-Riemannian manifolds $\left(B, g_{B}\right)$ (base) and $\left(F, g_{F}\right)$ (fiber) with warping function $f: B \rightarrow \mathbb{R}, f>0$, is the product manifold $B \times F$ endowed with the warped metric

$$
g=\pi_{B}^{*} g_{B}+f^{2} \pi_{F}^{*} g_{F},
$$

where $\pi_{B}, \pi_{F}$ are the natural projections of the product $B \times F$. The geodesic equations and elements of curvature of a warped product (expressed in the general semi-Riemannian setting) has been systematically studied by O'Neill 67, Chapter 7]. It is straightforward to check that if the base and fibers are complete and Riemannian then the warped product is complete, too (use Proposition 3.4), but this is not the case in the indefinite case, as it can be pointed out from the specially simple Beem and Buseman counterexample $\mathbb{R} \times_{f} \mathbb{R}, g=d x^{2}-e^{x} d y^{2}$. A careful study of geodesic completeness was carried out in [79, which will be our main reference. Extensions to other type of multiply warped manifolds can be found in 96. 97.

Warped completeness. A curve $\gamma=\left(\gamma_{B}, \gamma_{F}\right)$ in $B \times F$ is a geodesic if and only if it satisfies:

$$
\left\{\begin{aligned}
\frac{D \gamma_{B}^{\prime}}{d t} & =\frac{C}{\left(f \circ \gamma_{B}\right)^{3}} \nabla^{B} f \\
\frac{D \gamma_{F}^{\prime}}{d t} & =-\frac{2}{f \circ \gamma_{B}} \frac{d\left(f \circ \gamma_{B}\right)}{d t} \gamma_{F}^{\prime}
\end{aligned}\right.
$$

where $C=\left(f \circ \gamma_{B}\right)^{4} g_{F}\left(\gamma_{F}^{\prime}, \gamma_{F}^{\prime}\right)$ is necessarily a constant, $\nabla^{B}$ denotes the $g_{B^{-}}$ gradient, and $D / d t$ denotes the covariant derivative in the corresponding manifold. 
Remark 3.35. The equation for $\gamma_{F}$ implies that it is a pregeodesic for $g_{F}$ (recall (2.2)). As the equation for $\gamma_{B}$ is independent of $\gamma_{F}$, up to the constant $C$, one must study only an equation on $B$ and a reparameterization.

Now, taking into account the previous remark, the proof of Proposition 3.4 and some well-known facts (say, if a geodesic is continuously extendible to a point then it is also extendible as a geodesic (see 67. Lemma 5.8]) one easily has:

Lemma 3.36. If the fiber is complete, then for a geodesic $\gamma:[0, b) \rightarrow B \times F$, $b<+\infty$, the following properties are equivalent:

(1) $\gamma$ is extendible as a geodesic beyond $b$;

(2) $\gamma_{B}$ is continuously extendible beyond $b$;

(3) $\gamma_{B}^{\prime}$ lies in a compact subset of $T B$.

Moreover, if $g_{B}$ is Riemannian, previous conditions are also equivalent to:

(4) $\gamma_{B}$ lies in a compact subset of $B$.

From this result one can see that the role of the chosen fiber is irrelevant for the completeness of $\gamma$, except for the fact that, if it is Riemannian, the value of the constant $C$ in (3.7) is always positive. More precisely (see [79]):

Theorem 3.37. If the fiber of a warped product $B \times_{f} F$ is incomplete then the warped product is incomplete (and in the three causal senses, if it is indefinite).

If $\left(F, g_{F}\right)$ is complete and indefinite, the following assertions are equivalent:

(i) $B \times_{f} F$ is (resp. timelike, lightlike or spacelike) complete;

(ii) for any other complete and indefinite fiber $\left(F^{\prime}, g_{F^{\prime}}\right)$ the warped product $B \times_{f}$ $F^{\prime}$ is (resp. timelike, lightlike or spacelike) complete.

Definition 3.38. A triple $\left(B, g_{B}, f\right)$ is called (resp. timelike, lightlike or spacelike) warped complete if for any complete fiber $\left(F, g_{F}\right)$ the warped product $B \times F$ is (resp. timelike, lightlike or spacelike) complete.

More technically, any solution $\gamma_{B}$ of the differential equation (3.7) for $\left(B, g_{B}, f\right)$ will be called a (warped) geodesic projection and it will be called timelike, lightlike or spacelike depending on if the (necessarily constant) value of $D=g_{B}\left(\gamma_{B}^{\prime}, \gamma_{B}^{\prime}\right)+$ $C /\left(f \circ \gamma_{B}\right)^{2}$ is negative, 0 or positive. Theorem 3.37 can be paraphrased by saying that: a warped product with a complete indefinite fiber is (resp. timelike, lightlike or spacelike) complete if and only if all its (resp. timelike, lightlike or spacelike) geodesic projections are complete.

Remark 3.39. All the study will hold also if $B \times F$ is replaced by a fiber bundle $E(B, F)$ with base $B$ and fiber $F$, endowed with a metric as (3.6) in each trivializing neighborhood, where $\pi_{B}, \pi_{F}$ are now the natural projections of the fiber bundle. An example would be the one in Remark 3.11 when the connection $\omega$ is flat. 
Definite basis. Now, consider that the metric $g_{B}$ is Riemannian and $d$ is its distance. As the base of the warped product is totally geodesic, if $g_{B}$ is incomplete then $\left(B, g_{B}, f\right)$ is at least spacelike warped incomplete. Nevertheless, completeness in other causal senses may still hold. Now, we will focus in the case $g_{B}$ complete, and will consider an incomplete base in Subsection 3.7.

In the case that $B$ is compact, Lemma 3.36 (4) yields trivially the completeness. Otherwise, the behavior of $f$ at infinity belongs crucial. Fix a point $x_{0} \in B$ and define the continuous function $f_{\text {inf }}:[0,+\infty) \rightarrow \mathbb{R}$ so that

$$
f_{\text {inf }}(r)=\min \left\{f(x): d\left(x, x_{0}\right)=r\right\} .
$$

By using (3.7), the minimum increasing of the parameter $s$ of $\gamma_{B}$ when $d\left(\gamma_{B}(s), x_{0}\right)=$ $r$ can be bounded from below and, if $f$ is radial from $x_{0}$ (i.e., $f_{\text {inf }}=f$ ), also from above, one can obtain (see [79]):

Theorem 3.40. Let $\left(B, g_{B}\right)$ be Riemannian and complete. If $B$ is compact, then the triple $\left(B, g_{B}, f\right)$ is warped complete. Otherwise, $\left(B, g_{B}, f\right)$ is:

(a) warped complete if

$$
\int_{0}^{+\infty} \frac{f_{\text {inf }}}{\sqrt{1+f_{\text {inf }}^{2}}} d r=+\infty
$$

(b) timelike and lightlike complete if

$$
\int_{0}^{+\infty} f_{i n f} d r=+\infty
$$

(c) timelike complete if either $f_{\text {inf }}$ satisfies (3.9) or $f_{\text {inf }}$ is unbounded.

Moreover, if $f$ is radial, then the converses to (a), (b), (c) hold, too.

Remark 3.41. (1) The sufficient conditions in the items above satifies $(3.8) \Rightarrow$ (3.9) (and the latter implies the sufficient condition (c)) but the converses do not hold. Obviously, if inf $f(B)>0$ then the triple is warped complete.

(2) If we consider a "twisted product" , i.e., instead of a warping function $f$ in the definition of $g$, a twisting one $h: B \times F \rightarrow \mathbb{R}, h>0$, then none of previous results hold. In fact, take $B=F=S^{1}$ (the standard unit circumpherence in $\mathbb{C}$ ) and put $h\left(e^{i \theta_{1}}, e^{i \theta_{2}}\right)=e^{\sin \left(\theta_{1}-\theta_{2}\right)}$. A simple computation shows that the twisted product is incomplete (in fact, isometric to one of the $\mathcal{G}$ torus in Theorem 3.24).

Indefinite basis. If $\left(B, g_{B}\right)$ is indefinite then no such accurate results hold. In fact, there are even some open question which is related with other studied ones. As a first result obtained by combining the ideas for Theorems 3.10 3.40 we have:

Proposition 3.42. Let $\left(B, g_{B}\right)$ and indefinite manifold with $s$ conformal Killing vectors fields $\left\{K_{1}, \ldots, K_{s}\right\}$ satisfying the hypotheses in Theorem [3.10. For any smooth function $f$ on $B$ such that inf $f(B)>0$ and all $K_{i}(f)$ are bounded, the triple $\left(B, g_{B}, f\right)$ is warped complete. 
Remark 3.43. (1) The boundedness of $K_{i}(f)$ cannot be dropped, otherwise there are explicit counterexamples in [79, Counterexample 3.17]. This suggests that no accurate and general result on completeness such as Theorem 3.40 can be obtained for indefinite bases.

(2) Choose as base the natural Lorentzian torus $\mathbb{L}^{2} / \mathbb{Z}^{2}$ and $f(x, y)=e^{\sin (x-y)}$. The corresponding triple is warped complete as an application of Proposition 3.42 Nevertheless, any non-constant geodesic projection $\gamma_{B}$ with $x(t) \equiv y(t)$ is so that $\gamma_{B}^{\prime}$ is not contained in a compact subset of $T B$. Thus, choosing a compact fiber, an example of complete compact semi-Riemannian manifold with geodesics not contained in any compact subset of the tangent bundle is obtained (compare with Remarks 3.5 and $3.13(1)$ ).

Nevertheles, in spite of the first remark, the case of lightlike geodesics depends only on the bounds of $f$ and is related to the completeness of conformal metrics. Concretely, one can show:

Theorem 3.44. Let $\left(B, g_{B}\right)$ be semi-Riemannian manifold, $f \in C^{\infty}(M), f>0$.

(1) $\sup f(B)<+\infty$ and $\left(B, g_{B}, f\right)$ lightlike warped complete, imply $g_{B} / f^{2}$ complete.

(2) $\inf f(B)>0$ and $\left(B, g_{B}, f\right)$ not lightlike warped complete, imply $g_{B} / f^{2}$ not complete.

Remark 3.45. Consider the following open questions:

(Q1) A lightlike complete compact indefinite manifold is complete.

$(Q 2)$ Any triple $\left(B, g_{B}, f\right)$ with $\left(B, g_{B}\right)$ complete compact indefinite is warped complete.

(Q3) A complete compact indefinite manifold which is globally conformal to a complete one (in particular, to a Lorentzian spaceform) is complete.

As a consequence of Theorem 3.44 and the conformal invariance of lightlike completeness in the compact case, Theorem 2.2 we have:

$$
(Q 1) \quad \Longrightarrow \quad(Q 2) \quad \Longrightarrow \quad(Q 3) \text {. }
$$

As pointed out in Remark 3.27 there are tracks suggesting that $(Q 1)$ is not true. But the other questions (and, specially, (Q3) for Lorentzian spaceforms) remain open.

3.7. GRW spacetimes. Here, we apply previous results to a simple and important class of warped products, the GRW spacetimes. Those results allow one to prove the stability of completeness for the $C^{0}$ topology in the class of GRW spacetimes (see Theorem 3.48). We also study stability in the class of all the spacetimes, applying general results which generically yield $C^{1}$ stability.

Following [1, a Generalized Robertson-Walker (GRW) spacetime is a warped product $I \times{ }_{f} F$ with base $\left(I,-d t^{2}\right), I \subset \mathbb{R}$ interval, fiber a Riemannian manifold $\left(F, g_{F}\right)$ and any (positive) warping function $f$; with natural identifications $g=$ 
$-d t^{2}+f^{2}(t) g_{F}$. They generalize classical Friedman-Lemaitre-Robertson-Walker spacetimes because the fiber is not necessarily a model space. For relativistic motivations and several properties, see [1, 85, 87, and for some generalizations to more fibers, [83, 96, 97].

$C^{0}$ stability in the GRW class. Even though the base of a GRW spacetime may be non-complete, some simple considerations in addition to Theorem 3.40 allow one to characterize its completeness as follows.

Lemma 3.46. Let $I \times_{f} F$ be a GRW spacetime and fix $c \in I=(a, b)$. If $F$ is incomplete then the GRW is incomplete in the three causal senses. Otherwise:

1. The GRW is timelike complete if and only if

$$
\int_{a}^{c} \frac{f}{\sqrt{1+f^{2}}} d t=\int_{c}^{b} \frac{f}{\sqrt{1+f^{2}}} d t=+\infty .
$$

Otherwise, all the timelike geodesics (not tangent to I) are incomplete.

2. The GRW is lightlike complete if and only if

$$
\int_{a}^{c} f d t=\int_{c}^{b} f d t=+\infty
$$

Otherwise, all lightlike geodesics are incomplete.

3. The GRW is spacelike complete if and only if either $f$ satisfies (3.12) or, if

$$
\int_{a}^{c} f d t<+\infty \quad\left(\text { resp. } \int_{c}^{b} f d t<+\infty\right)
$$

then $f$ is unbounded in $(a, c)$ (resp. $(c, b))$.

Remark 3.47. (1) Clearly, the first integrals in (3.11) and (3.12) are related to the completeness of the causal geodesic $(t(s), x(s))$ towards the past direction $t(s) \searrow a$, and the second ones towards the future $t(s) \nearrow b$.

(2) Timelike $\Rightarrow$ lightlike $\Rightarrow$ spacelike completeness. There are counterexamples to the converses, but these converses do hold if $f$ is bounded.

Now, recall that: (i) both the complete and the incomplete Riemannian metrics on $F$ are $C^{0}$ open subsets of the set of all the metrics on $F$, and (ii) the conditions on $f$ which characterize causal completeness are both, open and closed in the set of all the positive warping functions on $I$. Therefore, one can prove that each one of the subsets of timelike, lightlike and spacelike geodesically complete GRW metrics on $I \times F$, are open and closed in the set $\mathcal{G R \mathcal { W }}(I \times F)$ of all the GRW metrics on $I \times F$. Summing up (see [85]):

Theorem 3.48. Both, completeness and incompleteness of each causal type, are $C^{r}$-stable for all $r \geq 0$ in the set $\mathcal{G} \mathcal{R} \mathcal{W}(I \times F)$. 
Finally, we remark that Lemma 3.46 not only characterizes incompleteness but also it says exactly which causal geodesics are incomplete: if $I \neq \mathbb{R}$ all timelike geodesics are incomplete either to the future or to the past, if $I=\mathbb{R}$ the geodesics tangent to the base are complete, but all the other timelike ones are either complete or incomplete, etc. So, if we call $C a u(M)$ the subset of the set of directions $S_{R} M$ (see Remark 3.6) which are causal, we have:

Proposition 3.49. In any GRW spacetime, the set of complete causal directions is a closed subset of $\mathrm{Cau}(M)$ (and $S_{R} M$ ). Thus, the incompleteness of causal geodesics is a stable property in the set of causal directions for a given GRW metric.

Remark 3.50. Nevertheless, in general the set of complete directions is not open in Cau $(M)$ or $S_{R}(M)$ (assume timelike incompleteness with $I=\mathbb{R}$ ) nor closed in $S_{R} M$ (take any spacelike complete non-lightlike complete GRW).

Stability in Lor(M). Recall that Theorem 3.48 only ensures the stability of completeness and incompleteness in the class of GRW spacetimes. We can wonder if the stability for a given GRW spacetime will hold in the class Lor $(M)$ of all the Lorentzian metrics on $M=I \times F$. This study (which can be carried out mainly for causal geodesics), is explained in detail in the book [1] Chapter 7], see also [9] 10. So, here we summarize the results very briefly. Generically, such results yield $C^{1}$ stability, as geodesic equations (Christoffel symbols) depend on the first derivatives of the metric tensor. Nevertheless, under a simplifying technical hypotheses on the fiber, the following $C^{0}$ result can be obtained.

Theorem 3.51. Let $I \times F$ be a GRW spacetime with homogeneous fiber $\left(F, g_{F}\right)$. Then timelike incompleteness is $C^{0}$ stable in $\operatorname{Lor}(M)$.

Proof. (Sketch). Firstly recall that all GRW spacetimes with complete fiber are globally hyperbolic, being the slices at constant $t$ Cauchy hypersurfaces ${ }^{6}$ (see 63 , Section 3.11]). Then, one takes an adapted auxiliary Riemannian norm, and estimates upper bounds for the increasing of the length of any timelike curve when each two such slices are crossed (so, some timelike geodesics will be of finite length).

For lightlike geodesics more accurate estimates are necessary and, so, $C^{1}$ stability would be needed. Nevertheless, it is possible to prove $C^{1}$ stability in the more general setting of non-partially imprisoned geodesics.

Theorem 3.52. Let $(M, g)$ be a semi-Riemannian manifold such that an inextendible geodesic $\gamma:(a, b) \rightarrow M$ exists which is: (i) forward-incomplete $(b<+\infty)$, and (ii) not partially imprisoned in a compact subset as $t \rightarrow b$.

Then there is a $C^{1}$-neighborhood $U(g)$ of $g$ such that all $g_{1} \in U(g)$ admits an incomplete geodesic of the same causal type of $\gamma$.

\footnotetext{
${ }^{6}$ In fact, any GRW spacetime is stably causal, as it admits the time function $t$. If $g_{F}$ is complete, then it is globally hyperbolic, because $t$ becomes a Cauchy time function.
} 
Now, notice that in all strongly causal spacetimes (in particular, any GRW), timelike and lightlike geodesics are never partially imprisoned ${ }^{7}$.

Corollary 3.53. In any GRW spacetime, both lightlike and timelike incompleteness are $C^{1}$ stable in $\operatorname{Lor}(M)$.

Finally, a natural assumption for the stability of causal completeness is the existence of a pseudoconvex nonspacelike geodesic system. This means that for each compact subset $K$ there is a second compact subset $H$ such that each causal geodesic with both endpoints in $K$ lies in $H$. Any globally hyperbolic spacetime contains such a system and, thus:

Theorem 3.54. Causal completeness is $C^{1}$ stable for any globally hyperbolic spacetime. In particular, this holds in any GRW spacetime with complete fiber $F$.

3.8. Stationary spacetimes. A connected Lorentzian manifold $(M, g)$ is called a stationary spacetime if it admits a timelike Killing vector field $K$. Implicitly, we will assume that one such stationary vector field $K$ has been chosen, and consider that it time-orientates the manifold. When the orthogonal distribution $K^{\perp}$ to $K$ is integrable, the spacetime is called static and admits a natural local warped structure associated to $K$. The completeness of stationary spacetimes has been already studied (see Proposition 3.8 Theorem 3.10). In Subsection 4.2 its geodesic connectedness will be studied in depth. Now, we will make some remarks on its structure and will deduce explicitly its geodesic equations.

Standard stationary spacetimes Let $\left(M_{0},\langle\cdot, \cdot\rangle_{R}\right)$ be a Riemannian manifold, and $\delta$ and $\beta$ a vector field and a positive smooth function on $M_{0}$, respectively. A standard stationary spacetime is the product manifold $M=\mathbb{R} \times M_{0}$ endowed with the Lorentz metric, under natural identifications:

$$
\langle\cdot, \cdot\rangle_{L}=-\beta(x) d t^{2}+\langle\cdot, \cdot\rangle_{R}+2\langle\delta(x), \cdot\rangle_{R} d t .
$$

When the cross term vanishes $(\delta \equiv 0)$ the spacetime is called standard static. This is a warped product with Riemannian base and negative definite fiber $M_{0} \times \sqrt{\beta} \mathbb{R}$.

Every stationary spacetime is locally a standard stationary one with $K=\partial_{t}$. Even more, any globally hyperbolic spacetime admits a spacelike Cauchy hypersurface $S$ ( 17 , see 63 ) and, if the spacetime is stationary with a complete Killing vector field $K$, i.e., $K$ has integral curves defined on the whole real line, moving $S$ by means of the flow of the Killing field $K$ one obtains (see [24, Theorem 2.3] for details):

Theorem 3.55. A globally hyperbolic stationary spacetime is a standard stationary one, if one of its timelike Killing vector fields $K$ is complete.

Nevertheless, if $K$ is static the spacetime may be non-standard static, as no Cauchy hypersurface orthogonal to $K$ may exist.

\footnotetext{
${ }^{7}$ Nevertheless, all spacelike geodesics may be imprisoned when $F$ is compact and $f$ is unbounded. In fact, this happens in de Sitter spacetime $\mathbb{S}_{1}^{n}$, which can be written as a warped product with fiber the round sphere and warping function cosh.
} 
Levi-Civita connection and geodesic equations From now on, $\nabla$ and $\nabla^{R}$ will denote the Levi-Civita connection of $\langle\cdot, \cdot\rangle_{L}$ and $\langle\cdot, \cdot\rangle_{R}$, respectively. For each vector field $V$ on $M_{0}, V \in \mathfrak{X}\left(M_{0}\right)$, its lifting to $M$ will be denoted $\bar{V}$ : that is, $\bar{V}_{(t, x)}=V_{x}$ for all $(t, x) \in M$ (analogously, if necessary, for a vector field on $\mathbb{R}$ ). Put

$$
\Lambda(x)=-\frac{1}{\beta(x)+\langle\delta(x), \delta(x)\rangle_{R}} \quad \text { for all } x \in M_{0} .
$$

An explicit computation from Koszul formula yields (see detailed computations for this section in [38):

$$
\begin{aligned}
\nabla_{\partial_{t}} \partial_{t}= & -\frac{1}{2} \Lambda\left\langle\delta, \nabla^{R} \beta\right\rangle_{R} \partial_{t}+\frac{1}{2} \Lambda\left\langle\delta, \nabla^{R} \beta\right\rangle_{R} \delta+\frac{1}{2} \nabla^{R} \beta \\
\nabla_{\bar{V}} \bar{W}= & \frac{1}{2} \Lambda\left(\left\langle W, \nabla_{V}^{R} \delta\right\rangle_{R}+\left\langle V, \nabla_{W}^{R} \delta\right\rangle_{R}\right) \partial_{t} \\
& -\frac{1}{2} \Lambda\left(\left\langle W, \nabla_{V}^{R} \delta\right\rangle_{R}+\left\langle V, \nabla_{W}^{R} \delta\right\rangle_{R}\right) \delta+\nabla_{V}^{R} W \\
2 \nabla_{\bar{V}} \partial_{t}= & 2 \nabla_{\partial_{t}} \bar{V}=-\Lambda\left(V(\beta)+\left\langle\delta, \nabla_{V}^{R} \delta\right\rangle_{R}-\left\langle\nabla_{\delta}^{R} \delta, V\right\rangle_{R}\right) \partial_{t} \\
& +\Lambda\left(V(\beta)+\left\langle\delta, \nabla_{V}^{R} \delta\right\rangle_{R}-\left\langle\nabla_{\delta}^{R} \delta, V\right\rangle_{R}\right) \delta+\nabla_{V}^{R} \delta-\left\langle\nabla_{(\cdot)}^{R} \delta, V\right\rangle_{R}^{\natural}
\end{aligned}
$$

for any $V, W \in \mathfrak{X}\left(M_{0}\right)$, where ${ }^{\natural}$ denotes the vector field on $M_{0}$ metrically associated to the corresponding 1-form (that is, $\left\langle Y,\left\langle\nabla_{(\cdot)}^{R} \delta, V\right\rangle_{R}^{\natural}\right\rangle_{R}=\left\langle\nabla_{Y}^{R} \delta, V\right\rangle_{R}$ for any $Y \in$ $\left.\mathfrak{X}\left(M_{0}\right)\right)$.

For the geodesic equations, recall that $q=\left\langle\gamma^{\prime}, \gamma^{\prime}\right\rangle_{L}$ is constant for any geodesic $\gamma(s)=(t(s), x(s)), s \in I$ and, as $\partial_{t}$ is a Killing vector field, $C_{\gamma}=\left\langle\gamma^{\prime}, \partial_{t}\right\rangle_{L}$ is constant, too. That is, one has the relations:

$$
\left\{\begin{array}{l}
\left\langle\left(t^{\prime}, x^{\prime}\right),\left(t^{\prime}, x^{\prime}\right)\right\rangle_{L}=-\beta(x)\left(t^{\prime}\right)^{2}+2\left\langle\delta(x), x^{\prime}\right\rangle_{R} t^{\prime}+\left\langle x^{\prime}, x^{\prime}\right\rangle_{R}=q \\
\left\langle\partial_{t},\left(t^{\prime}, x^{\prime}\right)\right\rangle_{L}=-\beta(x) t^{\prime}+\left\langle\delta(x), x^{\prime}\right\rangle_{R}=C_{\gamma}
\end{array}\right.
$$

Let $\mathfrak{X}_{r, s}\left(M_{0}\right)$ be the space of $r$-contravariant, $s$-covariant tensor fields on $M_{0}$ $\left(\mathfrak{X}\left(M_{0}\right) \equiv \mathfrak{X}_{1,0}\left(M_{0}\right) ; \mathfrak{X}_{1,1}\left(M_{0}\right)\right.$ is identifiable to the space of endomorphism fields). From the formulas of the connection, for the $x$ part one has:

$$
\nabla_{x^{\prime}}^{R} x^{\prime}=\left(t^{\prime}\right)^{2} R_{0}(x)+t^{\prime} R_{1}\left(x, x^{\prime}\right)+R_{2}\left(x,\left(x^{\prime}, x^{\prime}\right)\right)
$$

with $R_{0} \in \mathfrak{X}\left(M_{0}\right), R_{1} \in \mathfrak{X}_{1,1}\left(M_{0}\right), R_{2} \in \mathfrak{X}_{1,2}\left(M_{0}\right)$ so that:

$$
\left\{\begin{array}{l}
R_{0}(x)=-\frac{1}{2}\left(\Lambda\left\langle\delta, \nabla^{R} \beta\right\rangle_{R} \delta+\nabla^{R} \beta\right)(x) \\
R_{1}\left(x, x^{\prime}\right)=-\Lambda\left(\left\langle\nabla^{R} \beta, x^{\prime}\right\rangle_{R}+\operatorname{rot} \delta\left(x^{\prime}, \delta\right)\right) \delta(x)+\operatorname{rot} \delta\left(\cdot, x^{\prime}\right)^{\natural} \\
R_{2}\left(x,\left(x^{\prime}, \bar{x}^{\prime}\right)\right)=\Lambda \operatorname{Sym} \nabla^{R} \delta\left(x^{\prime}, \bar{x}^{\prime}\right) \delta(x) .
\end{array}\right.
$$

Remark 3.56. $R_{0}$ vanishes if $\beta$ is constant, $R_{1}$ reduces to $-\Lambda\left\langle\nabla^{R} \beta, x^{\prime}\right\rangle_{R} \delta(x)$ if $\delta$ is irrotational, and $R_{2}$ vanishes if $\delta$ is Killing.

By substituting in (3.15) the value of $t^{\prime}$ from the second equation in (3.14), a second order equation for the spacelike component $x(s)$ is obtained. Then, the first relation (3.14) can be regarded as a first integral of this equation. Moreover, a geodesic can be reconstructed for any solution of this differential equation. Summing up: 
Theorem 3.57. Consider a curve $\gamma(s)=(t(s), x(s)), s \in I \subset \mathbb{R}$ in a stationary spacetime $\left(\mathbb{R} \times M_{0},\langle\cdot, \cdot\rangle_{L}\right)$. The curve $\gamma$ is a geodesic if and only if $C_{\gamma}=\left\langle\gamma^{\prime}, \partial_{t}\right\rangle_{L}$ is a constant and, then, $x(s)$ satisfy

$$
\nabla_{x^{\prime}}^{R} x^{\prime}=\bar{R}_{0}(x)+\bar{R}_{1}\left(x, x^{\prime}\right)+\bar{R}_{2}\left(x, x^{\prime} \otimes x^{\prime}\right)
$$

where

$$
\begin{aligned}
& \bar{R}_{0}(x)=\frac{C_{\gamma}^{2}}{\beta^{2}(x)} R_{0}(x), \\
& \bar{R}_{1}\left(x, x^{\prime}\right)=-\frac{C_{\gamma}}{\beta(x)}\left(2 \frac{\left\langle\delta(x), x^{\prime}\right\rangle_{R}}{\beta(x)} R_{0}(x)+R_{1}\left(x, x^{\prime}\right)\right) \\
& \bar{R}_{2}\left(x, x^{\prime} \otimes x^{\prime}\right)=\frac{\left(\left\langle\delta(x), x^{\prime}\right\rangle_{R}\right)^{2}}{\beta^{2}(x)} R_{0}(x)+\frac{\left\langle\delta(x), x^{\prime}\right\rangle_{R}}{\beta(x)} R_{1}\left(x, x^{\prime}\right)+R_{2}\left(x,\left(x^{\prime}, x^{\prime}\right)\right),
\end{aligned}
$$

being $R_{0}, R_{1}, R_{2}$ as in 3.16).

Remark 3.58. Notice that for any solution $x(s)$ of (3.17), the second equation (3.14) yields the value of $t(s)$. Moreover, all the geodesics can be reparametrized in such a way that $C_{\gamma}=0,1$. Thus, we have the following two cases:

(a) Case $C_{\gamma}=0$. The geodesic $\gamma$ is always spacelike and orthogonal to $\partial_{t}$, and we can also assume that the value of $q$ is fixed equal to 1 . Recall that in this case $\bar{R}_{0}=\bar{R}_{1}=0$. In the static case, equation (3.17) is just the equation of the geodesics of $\left(M_{0},\langle\cdot, \cdot\rangle_{R}\right)$; thus, these geodesics can be regarded as trivial. Nevertheless, when $\delta$ is not null the differential equation becomes:

$$
\nabla_{x^{\prime}}^{R} x^{\prime}=\bar{R}_{2}\left(x, x^{\prime} \otimes x^{\prime}\right) .
$$

(b) Case $C_{\gamma}=1$. Equation (3.17) becomes rather complicated in general. In the static case, $x(s)$ satisfies the equation of a classical Riemannian particle under the potential $V=-1 / 2 \beta$, and the constant $q / 2$ is the classical energy (kinetic plus potential) of this particle. In the general case this interpretation does not hold, even though $q$ is a constant of the motion.

As we have seen, the direct study of the geodesic equations become extremely complicated, except if some simplifying assumptions are carried out. The simplest one is to consider the static case $\delta \equiv 0$, where previous equations reduces to a Riemannian dynamical system for $x$ and a reparametrization for $t$. Nevertheless, even in this case the reparametrization of $t$ may yield a non-trivial problem. In fact, in the problem of connecting by a geodesic two fixed endpoints $\left(t_{1}, x_{1}\right),\left(t_{2}, x_{2}\right)$, the existence of a solution $x(s)$ of (3.17) (which is simple to obtain in the static case) which connects $x_{1}, x_{2}$ yields a $t(s)$ which must be controlled in order to connect $t_{1}, t_{2}$. This makes necessary the variational approach in Subsection 4.2 below.

\section{Variational approaches in Lorentzian manifolds}

Due to the increasing knowledge in variational methods applicable to the study of critical points of unbounded functionals, at the end of 80's Benci, Fortunato, 
Giannoni and Masiello improved the investigations on the existence of geodesics in a Lorentzian manifold by means of variational tools. Next, we will describe the approach first for geodesics connecting two points in the Riemannian case, then in the two most characteristic Lorentzian cases: stationary (and globally hyperbolic) and orthogonal splitting.

4.1. The Riemannian framework for geodesics connecting two points. In order to apply global variational tools to the action functional $f$ as defined in 1.1. , some "good" abstract arguments and a suitable variational framework are required. We introduce both here in the Riemannian setting not only for completeness, but also for pedagogical reasons.

Let us remark that, in general, we are interested in the study of critical points of a $C^{1}$ functional $J$ defined on a Riemannian manifold $\Omega$, so a way to check the existence of critical levels is condition $(C)$ introduced by Palais in 68. Roughly speaking, it says that "each subset of $\Omega$ in which $J$ is bounded and its differential can tend to zero, has to have a critical point in its closure". But, starting from this definition, the true problem is to test the existence of such a critical point and a way to help in this "research" comes from the most used Palais-Smale condition.

Definition 4.1. Let $\Omega$ be a Riemannian manifold and $J \in C^{1}(\Omega, \mathbb{R})$. Functional $J$ satisfies the Palais-Smale condition on $\Omega$, briefly $(P S)$, if any sequence $\left(x_{k}\right)_{k} \subset \Omega$ such that

$$
\left(J\left(x_{k}\right)\right)_{k} \text { is bounded and } \lim _{k \rightarrow+\infty} J^{\prime}\left(x_{k}\right)=0
$$

has a subsequence converging in $\Omega$.

Thus, once that condition $(P S)$ is satisfied, a bounded-from-below functional has at least a critical point, its minimum. More precisely, a minimum theorem can be stated as follows (see, e.g, [76]).

Theorem 4.2. Let $\Omega$ be a complete Riemannian manifold and $J$ a $C^{1}$ functional on $\Omega$ which satisfies condition (PS). If $J$ is bounded from below then it attains its infimum.

The power of condition $(P S)$ is that not only it allows one to prove that an infimum is a minimum but also that more than one critical point can be found if the topology of $\Omega$ is rich enough. To this aim, different topological tools can be used but, here, for simplicity, we just introduce the Ljusternik-Schnirelman Theory and its main arguments (for more details, see, e.g., [2, 61, 69]).

Definition 4.3. Let $\Omega$ be a topological space. Given $A \subset \Omega$, the LjusternikSchnirelmann category of $A$ in $\Omega$, briefly $\operatorname{cat}_{\Omega}(A)$, is the least number of closed and contractible subsets of $\Omega$ covering $A$. If it is not possible to cover $A$ with a finite number of such sets, then $\operatorname{cat}_{\Omega}(A)=+\infty$.

For simplicity, we denote $\operatorname{cat}(\Omega)=\operatorname{cat}_{\Omega}(\Omega)$. 
Theorem 4.4. Let $\Omega$ be a Riemannian manifold and $J$ a $C^{1}$ functional on $\Omega$ which satisfies condition $(P S)$. Given any $k \in \mathbb{N}, k>0$, let us define

$$
c_{k}=\inf _{A \in \Gamma_{k}} \sup _{x \in A} J(x) \quad \text { with } \Gamma_{k}=\left\{A \subset \Omega: \operatorname{cat}_{\Omega}(A) \geq k\right\} .
$$

If $\Omega$ is complete, then $c_{k}$ is a critical value of $J$ for each $k$ such that $\Gamma_{k} \neq \emptyset$ and $c_{k} \in \mathbb{R}$. Moreover, if $J$ is bounded from below but not from above and cat $(\Omega)=+\infty$, then a sequence $\left(x_{k}\right)_{k}$ of critical points exists such that $\lim _{k \rightarrow+\infty} J\left(x_{k}\right)=+\infty$.

Remark 4.5. The same results of Theorems 4.2 and 4.4 still hold if the completeness of $\Omega$ is replaced by the completeness of each sublevel of $J$ or if condition $(P S)$ is replaced by weaker assumptions (see, e.g., [33] or also [93, pp. 80]).

Now, in order to introduce the correct functional framework (for more details, see, e.g., 68), if $(M, g)$ is a smooth $n$-dimensional connected semi-Riemannian manifold, let us define $H^{1}(I, M)$ the set of curves $z: I \rightarrow M$ such that for any local chart $(U, \varphi)$ of $M$, with $U \cap z(I) \neq \emptyset$, the curve $\varphi \circ z$ belongs to the Sobolev space $H^{1}\left(z^{-1}(U), \mathbb{R}^{n}\right)$. Taken $I=[0,1]$ (without loss of generality, as the set of geodesics is invariant by affine reparametrizations), it is well known that $H^{1}(I, M)$ is equipped with a structure of infinite dimensional manifold modelled on the Hilbert space $H^{1}\left(I, \mathbb{R}^{n}\right)$, as, if $z \in H^{1}(I, M)$, the tangent space to $H^{1}(I, M)$ at $z$ can be written as

$$
T_{z} H^{1}(I, M) \equiv\left\{\zeta \in H^{1}(I, T M): \pi \circ \zeta=z\right\},
$$

being $T M$ the tangent bundle of $M$ and $\pi: T M \rightarrow M$ the corresponding bundle projection. In other words, $T_{z} H^{1}(I, M)$ is the set of the vector fields along $z$ whose components with respect to a local chart are functions of class $H^{1}$.

It is easy to check that the action functional $f$ in (1.1) is well defined on $H^{1}(I, M)$; moreover, it is at least of class $C^{1}$ and there results

$$
f^{\prime}(z)[\zeta]=2 \int_{0}^{1} g(z(s))\left[z^{\prime}(s), \frac{D \zeta}{d s}\right] d s \quad \text { if } z \in H^{1}(I, M), \zeta \in T_{z} H^{1}(I, M) .
$$

Here in the following, we focus only on one model problem, for example the existence of geodesics joining two fixed points (as already remarked, different submanifolds have to be defined if other "boundary" conditions are required).

In this case, the "natural" setting of this variational problem is a suitable infinite dimensional submanifold of $H^{1}(I, M)$. More precisely, fixed $p, q \in M$, we can consider

$$
\Omega^{1}(p, q ; M)=\left\{z \in H^{1}(I, M): z(0)=p, z(1)=q\right\}
$$

such that, if $z \in \Omega^{1}(p, q ; M)$, the tangent space to $\Omega^{1}(p, q ; M)$ at $z$ is given by

$$
T_{z} \Omega^{1}(p, q ; M)=\left\{\zeta \in T_{z} H^{1}(I, M): \zeta(0)=0=\zeta(1)\right\} .
$$

Classical boot-strap arguments allow one to prove the following variational principle: 
Theorem 4.6. The curve $z: I \rightarrow M$ is a geodesic joining $p$ to $q$ in $M$ if and only if $z \in \Omega^{1}(p, q ; M)$ is a critical point of $f$ on $\Omega^{1}(p, q ; M)$.

Two particular subcases occur:

(i) $M \equiv \mathbb{R}$ is the 1 -dimensional Euclidean space;

(ii) $(M, g) \equiv\left(M_{0},\langle\cdot, \cdot\rangle_{R}\right)$ is a Riemannian manifold.

In case (i), fixed any $t_{p}, t_{q} \in \mathbb{R}$ the set defined in (4.2) becomes

$$
W^{1}\left(t_{p}, t_{q}\right)=\left\{t \in H^{1}(I, \mathbb{R}): t(0)=t_{p}, t(1)=t_{q}\right\}=H_{0}^{1}(I, \mathbb{R})+j^{*},
$$

with $H_{0}^{1}(I, \mathbb{R})=\left\{\tau \in H^{1}(I, \mathbb{R}): \tau(0)=0=\tau(1)\right\}$ vector space and

$$
j^{*}: s \in I \mapsto t_{p}+s \Delta_{t} \in \mathbb{R}, \quad \Delta_{t}=t_{q}-t_{p} .
$$

Whence, $W^{1}\left(t_{p}, t_{q}\right)$ is a closed affine submanifold of $H^{1}(I, \mathbb{R})$ with tangent space $T_{t} W^{1}\left(t_{p}, t_{q}\right) \equiv H_{0}^{1}(I, \mathbb{R})$ for all $t \in W^{1}\left(t_{p}, t_{q}\right)$.

On the other hand, in case (ii), if $M_{0}$ is a smooth enough Riemannian manifold (to our knowledge, at least of class $C^{3}$ ), by means of the Nash Embedding Theorem (see [66]) we can assume that $M_{0}$ is a submanifold of an Euclidean space $\mathbb{R}^{N}$ and $\langle\cdot, \cdot\rangle_{R}$ is the restriction to $M_{0}$ of the standard Euclidean metric of $\mathbb{R}^{N}$ with $d(\cdot, \cdot)$ the corresponding distance, i.e.,

$$
d\left(x_{1}, x_{2}\right)=\inf \left\{\int_{a}^{b} \sqrt{\left\langle\gamma^{\prime}, \gamma^{\prime}\right\rangle_{R}} d s: \gamma \in A_{x_{1}, x_{2}}\right\}
$$

with $x_{1}, x_{2} \in M_{0}$ and $\gamma \in A_{x_{1}, x_{2}}$ if $\gamma:[a, b] \rightarrow M_{0}$ is a piecewise smooth curve joining $x_{1}$ to $x_{2}$. Hence, it can be proved that manifold $H^{1}\left(I, M_{0}\right)$ is a submanifold of $H^{1}\left(I, \mathbb{R}^{N}\right)$ and can be identified with the set of the absolutely continuous curves $x: I \rightarrow \mathbb{R}^{N}, x(I) \subset M_{0}$, with square summable derivative. Thus, fixed any $x_{p}$, $x_{q} \in M_{0}$, the set defined in (4.2) can be identified as follows:

$$
\begin{aligned}
& \Omega^{1}\left(x_{p}, x_{q} ; M_{0}\right)=\left\{x: I \rightarrow M_{0}: x\right. \text { is absolutely continuous and such that } \\
& \left.\qquad x(0)=x_{p}, x(1)=x_{q}, \int_{0}^{1}\left\langle x^{\prime}, x^{\prime}\right\rangle_{R} d s<+\infty\right\} .
\end{aligned}
$$

Taken any $x \in \Omega^{1}\left(x_{p}, x_{q} ; M_{0}\right)$, let us point out that it is well defined

$$
\left\|x^{\prime}\right\|^{2}=\int_{0}^{1}\left\langle x^{\prime}, x^{\prime}\right\rangle_{R} d s
$$

and the tangent space in $x$ can be identified with

$$
\begin{aligned}
T_{x} \Omega^{1}\left(x_{p}, x_{q} ; M_{0}\right)=\{\xi: & I \rightarrow T M_{0}: \xi(s) \in T_{x(s)} M_{0} \text { for all } s \in I, \xi \text { is } \\
& \text { absolutely continuous and } \left.\xi(0)=0=\xi(1),\|\xi\|_{*}<+\infty\right\},
\end{aligned}
$$


being its Hilbert norm

$$
\|\xi\|_{*}^{2}=\int_{0}^{1}\left\langle\frac{D^{R} \xi}{d s}, \frac{D^{R} \xi}{d s}\right\rangle_{R} d s
$$

(here, $D^{R} / d s$ denotes the covariant derivative along $x$ relative to the Riemannian metric tensor $\left.\langle\cdot, \cdot\rangle_{R}\right)$.

Furthermore, let us point out that if $M_{0}$ is a complete Riemannian manifold with respect to $\langle\cdot, \cdot\rangle_{R}$, also $H^{1}\left(I, M_{0}\right)$ and $\Omega^{1}\left(x_{p}, x_{q} ; M_{0}\right)$ are complete Riemannian manifolds equipped with their scalar product.

The possibility to work in a submanifold of an Euclidean space $\mathbb{R}^{N}$ allowed Benci and Fortunato to prove a "splitting" lemma useful in the proof of condition $(P S)$ as it allows one to manage bounded sequences in $H^{1}\left(I, M_{0}\right)$ so to pass from a "weak" limit to a "strong" one (see [13, Lemma 2.1]).

Lemma 4.7. Let $\left(x_{k}\right)_{k} \subset \Omega^{1}\left(x_{p}, x_{q} ; M_{0}\right)$ be a sequence so that

$$
\left(\left\|x_{k}^{\prime}\right\|\right)_{k} \quad \text { is bounded. }
$$

Then, there exists $x \in H^{1}\left(I, \mathbb{R}^{N}\right)$ such that, up to subsequences, it is

$$
x_{k} \rightarrow x \text { weakly in } H^{1}\left(I, \mathbb{R}^{N}\right), \quad x_{k} \rightarrow x \text { uniformly in } I .
$$

If $M_{0}$ is complete, then $x \in \Omega^{1}\left(x_{p}, x_{q} ; M_{0}\right)$; furthermore, there exist two sequences $\left(\xi_{k}\right)_{k},\left(\nu_{k}\right)_{k} \subset H^{1}\left(I, \mathbb{R}^{N}\right)$ such that

$$
\begin{aligned}
& \xi_{k} \in T_{x_{k}} \Omega^{1}\left(x_{p}, x_{q} ; M_{0}\right), \quad x_{k}-x=\xi_{k}+\nu_{k} \quad \text { for all } k \in \mathbb{N}, \\
& \xi_{k} \rightarrow 0 \text { weakly and } \nu_{k} \rightarrow 0 \text { strongly in } H^{1}\left(I, \mathbb{R}^{N}\right) .
\end{aligned}
$$

At last, in this setting, we are able to estimate the Ljusternik-Schnirelman category of the space of curves $\Omega^{1}\left(x_{p}, x_{q} ; M_{0}\right)$ (cf. [36]).

Proposition 4.8. If the Riemannian manifold $M_{0}$ is not contractible in itself then $\Omega^{1}\left(x_{p}, x_{q} ; M_{0}\right)$ has infinite category and possesses compact subsets of arbitrarily high category.

Now, just in order to apply all the above arguments in a very simple case, let us consider $\left(M_{0},\langle\cdot, \cdot\rangle_{R}\right)$ smooth Riemannian manifold so that Nash Embedding Theorem holds. The well known Hopf-Rinow Theorem states that completeness implies geodesic connectedness (see [55] or also [11 pp. 4]), and it would be also a consequence of Weirstrass theorem for reflexive spaces (see, e.g., 93, Theorem $1.2])$. Here, we give an alternative proof by means of the variational setting and the topological arguments introduced in this section. Furthermore, such tools allow one to obtain also a multiplicity result under a minimum topological assumption (but which implies that the $\Omega^{1}\left(x_{p}, x_{q} ; M_{0}\right)$ does have a "rich" topology), the noncontractibility for $M_{0}$. This result cannot be deduced by more classical geometric methods (as those explained in Subsection [2.2), which allow to obtain just one (minimizing) geodesic in each homotopy class. 
Theorem 4.9. If $M_{0}$ is complete as metric space equipped with $d(\cdot, \cdot)$ in 4.3), then it is also geodesically connected, i.e., each couple of its points can be joined by a geodesic. Furthermore, if $M_{0}$ is not contractible in itself, then each couple of its points can be joined by infinitely many geodesics.

Proof. Our aim is to prove that, fixed $x_{p}, x_{q} \in M_{0}$, one or more geodesics exist joining $x_{p}$ to $x_{q}$ in $M_{0}$. Or better, by means of Theorem 4.6 it is enough to investigate the existence of critical points of the energy functional in (1.1) with $g=\langle\cdot, \cdot\rangle_{R}$, i.e.,

$$
f(x)=\int_{0}^{1}\left\langle x^{\prime}, x^{\prime}\right\rangle_{R} d s \quad \text { on } \Omega^{1}\left(x_{p}, x_{q} ; M_{0}\right) .
$$

In order to apply Theorem 4.2 we just need to prove that $f$ satisfies condition $(P S)$. So, let $\left(x_{k}\right)_{k} \subset \Omega^{1}\left(x_{p}, x_{q} ; M_{0}\right)$ be a sequence so that $\left(f\left(x_{k}\right)\right)_{k}$ is bounded and $f^{\prime}\left(x_{k}\right) \rightarrow 0$ as $k \rightarrow+\infty$. Clearly, by (4.4), (4.7) and the completeness of $M_{0}$, Lemma 4.7 applies and, up to subsequences, $x \in \Omega^{1}\left(x_{p}, x_{q} ; M_{0}\right)$ exists so that (4.5) holds. Moreover, $x_{k}-x \in H^{1}\left(I, \mathbb{R}^{N}\right)$ splits so that (4.6) is satisfied. Now, we have only to prove that $x_{k} \rightarrow x$ strongly in $H^{1}\left(I, \mathbb{R}^{N}\right)$; whence, to prove that $\xi_{k} \rightarrow 0$ strongly in $H^{1}\left(I, \mathbb{R}^{N}\right)$. But we know that $\xi_{k} \in T_{x_{k}} \Omega^{1}\left(x_{p}, x_{q} ; M_{0}\right)$, so, being $x_{k}=x+\xi_{k}+\nu_{k}$, from (4.1) and (4.6) if follows that $\varepsilon_{k} \searrow 0$ exists so that

$$
\varepsilon_{k}=\int_{0}^{1}\left\langle x_{k}^{\prime}, \xi_{k}^{\prime}\right\rangle_{R} d s=\int_{0}^{1}\left\langle x^{\prime}, \xi_{k}^{\prime}\right\rangle_{R} d s+\int_{0}^{1}\left\langle\xi_{k}^{\prime}, \xi_{k}^{\prime}\right\rangle_{R} d s+\int_{0}^{1}\left\langle\nu_{k}^{\prime}, \xi_{k}^{\prime}\right\rangle_{R} d s
$$

where it is

$$
\begin{aligned}
& \int_{0}^{1}\left\langle x^{\prime}, \xi_{k}^{\prime}\right\rangle_{R} d s \rightarrow 0 \quad \text { for the weak convergence } \xi_{k} \rightarrow 0, \\
& \int_{0}^{1}\left\langle\nu_{k}^{\prime}, \xi_{k}^{\prime}\right\rangle_{R} d s \rightarrow 0 \quad \text { for the strong convergence } \nu_{k} \rightarrow 0 .
\end{aligned}
$$

Thus, it has to be also

$$
\int_{0}^{1}\left\langle\xi_{k}^{\prime}, \xi_{k}^{\prime}\right\rangle_{R} d s \rightarrow 0
$$

hence, $\xi_{k} \rightarrow 0$ strongly in $H^{1}\left(I, \mathbb{R}^{N}\right)$. So, $x_{p}$ and $x_{q}$ must be joined by a geodesic which minimizes the (positive) energy functional $f$.

On the other hand, if $M_{0}$ is not contractible in itself, then the existence of infinitely critical points of $f$ in $\Omega^{1}\left(x_{p}, x_{q} ; M_{0}\right)$ is a direct consequence of Proposition 4.8 and Theorem 4.4

Remark 4.10. (1) Let us point out that the completeness is not a necessary condition for the geodesic connectedness. In fact, if $M_{0}$ is not complete but some convexity assumptions on its boundary are satisfied, then the geodesic connectedness still holds (see [7]).

(2) A similar approach works for the problem of the existence of a closed geodesic. Nevertheless, here the space of curves is formed by loops which are 
not attached to any point and, so, one needs either the compactness of the manifold or some assumption at infinity, in order to ensure Palais Smale condition (e.g., see [16, 27 for two different sets of hypotheses which overcome the lack of compactness, or the careful discussion in [8]). Moreover, a topological hypothesis (as compactness) is also needed even for the problem of the existence of one closed geodesic: notice that the minimum critical points of the energy functional are always constant geodesics which cannot be accepted as solutions of the problem. As far as we know, the general multiplicity problem remains open (see [51]). The difficulty appears because infinite critical points of the functional for closed geodesics may mean a single closed geodesic which is run many times. In fact, topological assumptions which implied the existence of critical points with arbitrary lengths of $f$ yielded multiplicity of solutions in the case of geodesic connectedness, but in the case of closed geodesics only ensure the existence of a non-trivial one.

\subsection{Variational principles for static and stationary space-} times: extrinsic and intrinsic approaches. Differently from the Riemannian case, for Lorentzian manifolds something like Theorem 4.9 does not hold. In fact, a counterexample is given by the anti-de Sitter spacetime $M=]-\frac{\pi}{2}, \frac{\pi}{2}[\times \mathbb{R}$ equipped with the Lorentzian metric

$$
\langle\cdot, \cdot\rangle_{L}=\frac{1}{\cos ^{2} x}\left(-d t^{2}+d x^{2}\right)
$$

which is geodesically complete (and static), but not geodesically connected (cf. [71]). Moreover, in a Lorentzian manifold the minimizing arguments in Theorem 4.2 cannot be used directly as the action functional is strongly unbounded both from below and from above.

Anyway, in some model problems alternatives are possible. And, in fact, two different techniques can be used for stationary spacetimes:

(i) the extrinsic approach, when the Lorentzian manifold "splits" in a Riemannian manifold and an Euclidean space and the metric coefficients are "time independent" (see, e.g., 14, 23, 29]);

(ii) the intrinsic one, when such a splitting is not given "a priori" but a timelike Killing vector field exists anyway (see [24, 30, 49]).

Even though the latter has revealed as the most powerful [24, in this section, we want to outline both these techniques, showing the main used tools and their limits.

Case (i) (Extrinsic). The main idea in this case is transforming the indefinite problem in a subtler problem on a Riemannian manifold. In order to give an idea of this way of work, let us consider the simplest model of Lorentzian manifolds of this kind. Recall from Subsection 3.8 that, explicitly, a standard static spacetime is a connected Lorentzian manifold $\left(M,\langle\cdot, \cdot\rangle_{L}\right)$ with $M=\mathbb{R} \times M_{0}$ and

$$
\langle\cdot, \cdot\rangle_{L}=-\beta(x) d t^{2}+\langle\cdot, \cdot\rangle_{R},
$$


for a Riemannian manifold $\left(M_{0},\langle\cdot, \cdot\rangle_{R}\right), t$ natural coordinate of $\mathbb{R}, x \in M_{0}$ and a smooth positive function $\beta: M_{0} \rightarrow \mathbb{R}$.

Remark 4.11. Recall that, essentially, the study of geodesic connectedness in the standard case is equivalent to that one on any static spacetime by using the universal covering (see 90, Theorem 2.1] and [5, Section 2]). This does not hold by any means in the stationary case. In fact, as far as we know, it is not known if a compact stationary spacetime must be geodesically connected. And this problem cannot be reduced to the standard one by passing through the universal covering (the 3-sphere admits a stationary metric, see for example [79]).

Fixed $p=\left(t_{p}, x_{p}\right), q=\left(t_{q}, x_{q}\right) \in M$, thanks to this splitting we have that the manifold of curves joining $p$ to $q$ splits, also, so it is

$$
\Omega^{1}(p, q ; M) \equiv W^{1}\left(t_{p}, t_{q}\right) \times \Omega^{1}\left(x_{p}, x_{q} ; M_{0}\right)
$$

and, consequently, also its tangent space in each $z=(t, x) \in \Omega^{1}(p, q ; M)$ becomes

$$
T_{z} \Omega^{1}(p, q ; M) \equiv H_{0}^{1}(I, \mathbb{R}) \times T_{x} \Omega^{1}\left(x_{p}, x_{q} ; M_{0}\right) .
$$

Hence, the action functional $f$ in (1.1) becomes

$$
f(z)=\int_{0}^{1}\left\langle z^{\prime}, z^{\prime}\right\rangle_{L} d s=\int_{0}^{1}\left(-\beta(x)\left(t^{\prime}\right)^{2}+\left\langle x^{\prime}, x^{\prime}\right\rangle_{R}\right) d s
$$

if $z=(t, x) \in \Omega^{1}(p, q ; M)$, with Fréchet differential

$$
f^{\prime}(z)[\zeta]=-\int_{0}^{1}\left(\beta^{\prime}(x)[\xi]\left(t^{\prime}\right)^{2}+2 \beta(x) t^{\prime} \tau^{\prime}\right) d s+2 \int_{0}^{1}\left\langle x^{\prime}, \frac{D^{R} \xi}{d s}\right\rangle_{R} d s
$$

for all $\zeta=(\tau, \xi) \in T_{z} \Omega^{1}(p, q ; M)$, where $\beta^{\prime}(x)[\xi]$ denotes the differential of $\beta$ on $\xi$ at $x \in M_{0}$.

As Benci, Fortunato and Giannoni showed in their pioneer work 14, Theorem 2.1], the following new variational principle can be stated.

Proposition 4.12. Let $z^{*}=\left(t^{*}, x^{*}\right) \in \Omega^{1}(p, q ; M)$. The following statements are equivalent:

(a) $z^{*}$ is a critical point of the action functional $f$ defined as in 4.9);

(b) $x^{*}$ is a critical point of functional $\mathcal{J}: \Omega^{1}\left(x_{p}, x_{q} ; M_{0}\right) \rightarrow \mathbb{R}$ defined as

$$
\mathcal{J}(x)=\int_{0}^{1}\left\langle x^{\prime}, x^{\prime}\right\rangle_{R} d s-\Delta_{t}^{2}\left(\int_{0}^{1} \frac{1}{\beta(x)} d s\right)^{-1}
$$

and $t^{*}=\Psi\left(x^{*}\right)$, with $\Delta_{t}^{2}=\left(t_{q}-t_{p}\right)^{2}$ and $\Psi: \Omega^{1}\left(x_{p}, x_{q} ; M_{0}\right) \rightarrow W^{1}\left(t_{p}, t_{q}\right)$ such that

$$
\Psi(x)(s)=t_{p}+\Delta_{t}\left(\int_{0}^{1} \frac{1}{\beta(x)} d s\right)^{-1} \int_{0}^{s} \frac{1}{\beta(x(\sigma))} d \sigma .
$$


Moreover, $f\left(z^{*}\right)=J\left(x^{*}\right)$.

Proof. The main idea of this proof is to introduce the partial derivatives

$$
\frac{\partial f}{\partial t}(z)[\tau]=f^{\prime}(z)[(\tau, 0)], \quad \frac{\partial f}{\partial x}(z)[\xi]=f^{\prime}(z)[(0, \xi)],
$$

for any $z=(t, x) \in \Omega^{1}(p, q ; M), \tau \in H_{0}^{1}(I, \mathbb{R}), \xi \in T_{x} \Omega^{1}\left(x_{p}, x_{q} ; M_{0}\right)$, and consider the kernel of $\frac{\partial f}{\partial t}$, i.e.,

$$
\mathcal{N}=\left\{z \in \Omega^{1}(p, q ; M): \frac{\partial f}{\partial t}(z) \equiv 0\right\} .
$$

Once proved that $z=(t, x) \in \mathcal{N}$ if and only if $t=\Psi(x)(\mathcal{N}$ is the graph of the $C^{1}$ map $\left.\Psi\right)$, then it is enough to define $\mathcal{J}=\left.f\right|_{\mathcal{N}}$, i.e., $\mathcal{J}(x)=f(\Psi(x), x)$ for all $x \in \Omega^{1}\left(x_{p}, x_{q} ; M_{0}\right)$, and to remark that

$$
\mathcal{J}^{\prime}(x)[\xi]=f^{\prime}(\Psi(x), x)[(0, \xi)] \text { for all } \xi \in T_{x} \Omega^{1}\left(x_{p}, x_{q} ; M_{0}\right) .
$$

Now, we can investigate the existence of critical points of $\mathcal{J}$ on $\Omega^{1}\left(x_{p}, x_{q} ; M_{0}\right)$.

It is quite easy to prove that this new functional is bounded from below if coefficient $\beta$ is bounded from above (see [14]), but by means of a reduction to a 1-dimensional problem (following some ideas introduced in 22]), very careful estimates on the diverging sequences of $\Omega^{1}\left(x_{p}, x_{q} ; M_{0}\right)$ allow one to prove the following result (for all the details, see [5. Proposition 4.1]).

Proposition 4.13. If coefficient $\beta$ grows at most quadratically at infinity, i.e., there exist $\lambda \geq 0, \mu, k \in \mathbb{R}, \alpha \in[0,2)$ and a point $\bar{x} \in M_{0}$ such that ${ }^{8}$

$$
0<\beta(x) \leq \lambda d^{2}(x, \bar{x})+\mu d^{\alpha}(x, \bar{x})+k \quad \text { for all } x \in M_{0},
$$

then functional $\mathcal{J}$ is

- bounded from below;

- coercive, i.e.,

$$
\mathcal{J}(x) \rightarrow+\infty \quad \text { if } \quad\left\|x^{\prime}\right\| \rightarrow+\infty .
$$

Thus, if (4.10) holds, not only $\mathcal{J}$ has an infimum on $\Omega^{1}\left(x_{p}, x_{q} ; M_{0}\right)$ but any sequence $\left(x_{k}\right)_{k} \subset \Omega^{1}\left(x_{p}, x_{q} ; M_{0}\right)$, such that $\left(\mathcal{J}\left(x_{k}\right)\right)_{k}$ is bounded and $\mathcal{J}^{\prime}\left(x_{k}\right) \rightarrow 0$, has to be bounded and Lemma 4.7 can apply. Then, by suitable computations (for more details, see, e.g., [5 Proposition 4.3]), we are able to prove that:

Proposition 4.14. In the hypotheses of Proposition 4.13 functional $\mathcal{J}$ satisfies condition $(P S)$ in $\Omega^{1}\left(x_{p}, x_{q} ; M_{0}\right)$.

\footnotetext{
${ }^{8}$ Obviously, one can put $\mu=0$ without loss of generality here (as well as in formula 4.11 below one can put $\left.\mu_{1}=0\right)$. Nevertheless, in this fashion, one can compare better this result with the previously obtained ones.
} 
At last, from Propositions 4.13 and 4.14 and Theorem 4.2 respectively 4.4 it follows the following result (see [5] Theorem 1.1]).

Theorem 4.15. Let $\left(M,\langle\cdot, \cdot\rangle_{L}\right)$ be a standard static Lorentzian manifold with $M=$ $\mathbb{R} \times M_{0}$ and $\langle\cdot, \cdot\rangle_{L}$ as in $(4.8)$. If the smooth Riemannian manifold $\left(M_{0},\langle\cdot, \cdot\rangle_{R}\right)$ is complete and coefficient $\beta$ in (4.8) satisfies the at most quadratic growth condition (4.10), then, $M$ is geodesically connected.

Furthermore, if $M_{0}$ is non-contractible in itself, then any two points can be joined by a sequence of (spacelike) geodesics $\left(z_{k}\right)_{k}$ with diverging lengths.

Let us point out that this existence result can be extended also to a non complete static manifold but under suitable hypotheses on its boundary (see 5. Section $6]$ and references therein) while the growth assumption on $\beta$ cannot be further improved as there are counterexamples to geodesic connectedness if $\beta$ grows more than quadratically at infinity.

Example 4.16. For fixed $\varepsilon>0$, consider the static spacetime

$$
\left(\mathbb{R}^{2},\langle\cdot, \cdot\rangle_{L}\right), \quad\langle\cdot, \cdot\rangle_{L}=-\beta_{\varepsilon}(x) d t^{2}+d x^{2}
$$

where $\beta_{\varepsilon}(x)$ is a smooth function such that

$$
\left\{\begin{array}{l}
\beta_{\varepsilon}(x)=1+|x|^{2+\varepsilon} \quad \text { if } \quad x \in \mathbb{R} \backslash(-1,1) \\
\beta_{\varepsilon}([-1,1]) \subset[1,2] .
\end{array}\right.
$$

It can be proved that a couple of points $p, q \in \mathbb{R}^{2}$ exists which cannot be joined by means of a geodesic (for all the details, see [5, Section 7]).

Remark 4.17. Careful estimates allow one to investigate also the number of timelike geodesics joining $p$ to $q$ according to $\left|\Delta_{t}\right| \nearrow+\infty$ (see, e.g., 5] Section 5] and the related references).

Similar arguments to those ones outlined for the standard static spacetimes, can be also used for the study of geodesic connectedness for the larger class of standard stationary spacetimes according to the definition in (3.13).

In fact, fixed $p=\left(t_{p}, x_{p}\right), q=\left(t_{q}, x_{q}\right) \in M$, also in this setting a suitable new variational principle can be proved (see [47. Theorem 2.2] or also 61, Theorem 3.3.2]) so to define a new "Riemannian" functional

$$
\begin{aligned}
\mathcal{J}_{1}(x) & =\int_{0}^{1}\left\langle x^{\prime}, x^{\prime}\right\rangle_{R} d s+\int_{0}^{1} \frac{\left\langle\delta(x), x^{\prime}\right\rangle_{R}^{2}}{\beta(x)} d s \\
& -\left(\int_{0}^{1} \frac{\left\langle\delta(x), x^{\prime}\right\rangle_{R}}{\beta(x)} d s-\Delta_{t}\right)^{2}\left(\int_{0}^{1} \frac{1}{\beta(x)} d s\right)^{-1}
\end{aligned}
$$

on $\Omega^{1}\left(x_{p}, x_{q} ; M_{0}\right)$ which is bounded from below and coercive under "good" growth hypotheses on coefficients $\beta$ and $\delta$ in (3.13). Thus, with this extrinsic approach, also in this case the following result can be stated (for all the details, see 4 , Theorem 1.2]). 
Theorem 4.18. Let $M=\mathbb{R} \times M_{0}$ be a standard stationary spacetime as in (3.13). Suppose that the smooth Riemannian manifold $\left(M_{0},\langle\cdot, \cdot\rangle_{R}\right)$ is complete and there exists a point $\bar{x} \in M_{0}$ such that coefficient $\beta$ satisfies the at most quadratic growth condition (4.10) and $\delta$ the at most linear

$$
\sqrt{\langle\delta(x), \delta(x)\rangle_{R}} \leq \lambda_{1} d(x, \bar{x})+\mu_{1} d^{\alpha_{1}}(x, \bar{x})+k_{1} \quad \text { for all } x \in M_{0}
$$

for suitable $\lambda_{1} \geq 0, \mu_{1}, k_{1} \in \mathbb{R}, \alpha_{1} \in[0,1)$. Then, $M$ is geodesically connected.

Furthermore, if $M_{0}$ is non-contractible in itself, then any two points can be joined by a sequence of (spacelike) geodesics $\left(z_{k}\right)_{k}$ with diverging lengths.

Clearly, also in this case the growth hypothesis on $\beta$ cannot be improved (see the previous Example 4.16). Morevoer, if $\delta$ has a more than linear growth, we are able to find an example (see [4. Example 2.7]) whose functional $\mathcal{J}_{1}$ is unbounded from below; so, in general, one does not expect geodesic connectedness for this case.

Remark 4.19. Also in stationary spacetimes it is possible to give an estimate of the number of timelike geodesics connecting two fixed points (see, e.g., 61 Subsection 3.5]); moreover, the completeness assumption can be weakened (for a quite complete discussion on this subject, see [3]).

At last let us point out that, while in the static case it is equivalent to investigate the geodesic connectedness both in the standard and in the general case, the study of the standard stationary case hides an important fact: the same spacetime can split as (3.13) in very different ways (with very different $\beta, \delta$ ) because just one such splitting is not intrinsic to the spacetime. As a simple and extreme example, Minkowski spacetime can be written as (3.13) either with an arbitrary growth of $\delta$ or with an incomplete $\langle\cdot, \cdot\rangle_{R}$ (see 24 Appendix A.2]). More deeply, the bounds (4.10) for $\beta$ and (4.11) for $\delta$ do not have a geometric meaning on $M$, except as sufficient (but neither necessary nor intrinsic) conditions for global hyperbolicity (see [82]).

Case (ii) (Intrinsic). Giannoni and Piccione 49] introduced a different intrinsic approach to the study of geodesic connectedness in a stationary spacetime whose definition does not make use of any global splitting.

Recall from (3.1) that if $z: I \rightarrow M$ is a $C^{1}$ curve and $K$ is a Killing vector field on $M$, then it is

$$
\left\langle z^{\prime}, \frac{D}{d s} K(z)\right\rangle_{L} \equiv 0 \quad \text { on } I
$$

and, if $z$ is only absolutely continuous, this holds almost everywhere in $I$. In particular, if $z$ is a geodesic this property implies the existence of a constant $C_{z} \in \mathbb{R}$ such that

$$
\left\langle z^{\prime}, K(z)\right\rangle_{L} \equiv C_{z} \quad \text { for all } s \in I .
$$

Thus, if $\left(M,\langle\cdot, \cdot\rangle_{L}\right)$ is a stationary spacetime, (4.12) gives a natural constraint to the action functional $f$ defined in (1.1). More precisely, for each $p, q \in M$, we 
can define

$$
\begin{aligned}
C_{K}^{1}(p, q)=\left\{z \in C^{1}(I, M):\right. & z(0)=p, z(1)=q, \text { and } \\
& \left.\exists C_{z} \in \mathbb{R} \text { such that }\left\langle z^{\prime}, K(z)\right\rangle_{L} \equiv C_{z}\right\}
\end{aligned}
$$

and the following variational principle can be stated (see [49, pp. 2] joint to some arguments developed in [49, Proof of Theorem 3.3]).

Theorem 4.20. If $z \in C_{K}^{1}(p, q)$ is a critical point of $f$ restricted to $C_{K}^{1}(p, q)$, then $z$ is a geodesic connecting $p$ to $q$.

Even if functional $f$ is defined in $C_{K}^{1}(p, q)$, it cannot be managed only in this space, as this space is "too small" for problems of convergence. So, the "natural" setting of this variational problem is $\Omega^{1}(p, q ;, M)$ and the corresponding constraint is

$$
\begin{aligned}
\Omega_{K}^{1}(p, q)=\left\{z \in \Omega^{1}(p, q ; M):\right. & \exists C_{z} \in \mathbb{R} \text { such that } \\
& \left.\left\langle z^{\prime}, K(z)\right\rangle_{L}=C_{z} \text { a.e. on }[0,1]\right\} .
\end{aligned}
$$

It is quite simple to check that the closure of $C_{K}^{1}(p, q)$ with respect to the $H^{1}$ norm is a subset of $\Omega_{K}^{1}(p, q)$; furthermore, Theorem 4.20 can be reformulated as follows (see [49, Theorem 3.3]).

Theorem 4.21. If $z \in \Omega_{K}^{1}(p, q)$ is a critical point of $f$ restricted to $\Omega_{K}^{1}(p, q)$, then $z$ is a geodesic connecting $p$ and $q$.

Thus, in order to find sufficient conditions for the existence of critical points of $f$ on $\Omega_{K}^{1}(p, q)$, let us introduce the following definitions.

Definition 4.22. Fixed $c \in \mathbb{R}$ the set $\Omega_{K}^{1}(p, q)$ is c-precompact if every sequence $\left(z_{k}\right)_{k} \subset \Omega_{K}^{1}(p, q)$ with $f\left(z_{k}\right) \leq c$ has a subsequence which converges weakly in $\Omega^{1}(p, q ; M)$ (hence, uniformly in $M$ ). Furthermore, the restriction of $f$ to $\Omega_{K}^{1}(p, q)$ is pseudo-coercive if $\Omega_{K}^{1}(p, q)$ is $c$-precompact for all $c \geq \inf f\left(\Omega_{K}^{1}(p, q)\right)$.

Then, the geodesic connectivity between each $p$ and $q$ will be a consequence of the following theorem (see [49, Theorem 1.2]).

Theorem 4.23. If $\Omega_{K}^{1}(p, q)$ is not empty and there exists $c>\inf f\left(\Omega_{K}^{1}(p, q)\right)$ such that $\Omega_{K}^{1}(p, q)$ is c-precompact, then there exists at least one geodesic joining $p$ to $q$ in $M$.

Moreover, if in the stationary spacetime $M$ there exists a Killing vector field $K$ which is complete, a multiplicity result can be stated (see [49, Theorem 1.3]).

Theorem 4.24. Let $\Omega_{K}^{1}(p, q)$ be not empty and assume that $f$ is pseudo-coercive in $\Omega_{K}^{1}(p, q)$. Then, if $K$ is complete and $M$ is non-contractible in itself, any two points can be joined by a sequence of (spacelike) geodesics $\left(z_{k}\right)_{k}$ with diverging critical levels $f\left(z_{k}\right) \nearrow+\infty$. 
Thus, reduced the geodesic connectedness problem to the research of "good" hypotheses which guarantee the application of Theorem 4.23 the explicit example pointed out in [49, Appendix A] is a standard stationary spacetime $M=\mathbb{R} \times M_{0}$ equipped with metric (3.13) whose coefficient $\beta$ has to be bounded from above and far away from zero while $\delta$ must have a sublinear growth, i.e., (4.11) holds with $\lambda_{1}=0$.

Let us point out that the main limitation of Giannoni and Piccione's results is that pseudo-coercivity condition is analytical and very technical. In fact, it can be regarded as a tidy and neat version of Palais-Smale condition for the stationary ambient. Furthermore, in general, the assumption $\Omega_{K}^{1}(p, q)$ non-empty must be imposed. Nevertheless, the possibility of $\Omega_{K}^{1}(p, q)=\emptyset$ can be ruled out if $K$ is complete (compare with [49, Lemma 5.7] and [24, Proposition 3.6]).

Proposition 4.25. If the timelike Killing vector field $K$ is complete, then it is $\Omega_{K}^{1}(p, q) \neq \emptyset$ for each $p, q \in M$.

Now, we want to translate the technical condition of pseudo-coercivity in terms of the (Lorentzian) geometry of the manifold (for a complete proof, see [24] Section $5])$.

Proposition 4.26. Let $\left(M,\langle\cdot, \cdot\rangle_{L}\right)$ be a stationary spacetime with a complete timelike Killing vector field $K$ and a complete smooth spacelike Cauchy hypersurface $\mathcal{S}$. Then, the restriction of action function $f$ to $\Omega_{K}^{1}(p, q)$ is pseudo-coercive for any $p, q \in M$.

Thus, by means of Theorems 4.234 .24 and Propositions 4.25 4.26 the following results can be stated.

Theorem 4.27. Let $\left(M,\langle\cdot, \cdot\rangle_{L}\right)$ be a stationary spacetime with a complete timelike Killing vector field $K$. If $M$ is globally hyperbolic with a complete (smooth, spacelike) Cauchy hypersurface $\mathcal{S}$, then it is geodesically connected.

Furthermore, if $M$ is non-contractible in itself, then any two points can be joined by a sequence of (spacelike) geodesics $\left(z_{k}\right)_{k}$ such that $f\left(z_{k}\right) \nearrow+\infty$.

Let us point out that, if $M$ has a complete timelike Killing vector field $K$ and is globally hyperbolic with a complete spacelike Cauchy hypersurface $\mathcal{S}$, from Theorem [3.55 it follows that the spacetime is the product $\mathbb{R} \times \mathcal{S}$, and its metric can be written as in (3.13) for a certain vector field $\delta$ on $\mathcal{S}$ and the identifications

$$
\begin{gathered}
K(z) \equiv(0,1) \in \mathbb{R} \times T_{x} \mathcal{S} \quad \text { for all } z=(t, x) \in M(t \in \mathbb{R}, x \in \mathcal{S}), \\
\langle K(z), K(z)\rangle_{L}=-\beta(x) \quad \text { for all } \quad z=(t, x) \in M .
\end{gathered}
$$

Nevertheless, neither $K$ nor $\mathcal{S}$ are unique and this global splitting is not canonically associated to a spacetime even under the hypotheses of Theorem 4.27 Anyway, the results will be independent of the chosen $K, \mathcal{S}$ and no growth hypothesis on $\beta$, $\delta$ is required.

Vice versa, if $M=\mathbb{R} \times M_{0}$ is a standard stationary spacetime with metric (3.13) and the Riemannian metric $\langle\cdot, \cdot\rangle_{R}$ on $M_{0}$ is complete while $\beta$, respectively 
$\delta$, satisfies (4.10), respectively (4.11), then $M$ is globally hyperbolic with $\{0\} \times M_{0}$ complete Cauchy hypersurface. Moreover, the standard timelike Killing vector field $K=\partial_{t}$ is complete. Thus, Theorem 4.18 follows from Theorem 4.27

Let us remark that both completeness of the Killing vector field $K$ and the completeness of the Cauchy hypersurface $\mathcal{S}$ are needed in the proof of Theorem 4.27 as counterexamples to geodesic connectedness can be found if one of these conditions is dropped (see 24, Appendix A.3]).

At last, let us summary the results of geodesic connectedness in a stationary spacetime $\left(M,\langle\cdot, \cdot\rangle_{L}\right)$ :

\begin{tabular}{|c|c|c|}
\hline Hypotheses Thm 4.15 (static) & & \\
\hline$U$ & & \\
\hline Extrinsic Hyp. Thm 4.18 & $\Longrightarrow$ & Geometric Hyp. Thm 4.27 \\
\hline & & $\Downarrow$ \\
\hline$\Downarrow$ extrinsic & & Technical Hyp. Thm 4.23 \\
\hline & & $\Downarrow$ intrinsic \\
\hline
\end{tabular}

And, as widely discussed in [24, the intrinsic geometric hypotheses on the stationary spacetime in Thm. 4.27 are essentially equivalent to the technical analitycal hypotheses on the space of curves in Thm. 4.23 .

4.3. Time dependent metrics and saddle critical points. Now, we want to take care of models of Lorentzian manifolds whose metric is timedependent so it is necessary to study directly the corresponding strongly indefinite action functional $f$ defined in (1.1). Such an approach is based essentially on a Galerkin finite-dimensional approximation of the "real part" $W^{1}\left(t_{p}, t_{q}\right)$ and yields estimates for the connectedness of very general manifolds, as the orthogonal splitting ones. In this setting, the existence of at least one connecting geodesic is obtained by means of Rabinowitz's Saddle Point Theorem while multiplicity results follow from the Relative Category Theory.

The basic ideas of this method were introduced in [13], in order to study the geodesic connectedness in a standard stationary spacetime, then they were applied to the splitting case in [15, 48, 61, 72]. Anyway, some details were completely analyzed later on (see 25, 26]) and, even if such results follow from [26] Theorems 1.5 and 1.10], here, they are presented for the first time in a self-contained presentation.

First of all, let us introduce the main tools of the abstract theorems we need for dealing with unbounded functionals.

In order to obtain the existence of at least one solution, let us state the following slight variant of the classical Saddle Point Theorem (cf. [15, 76] or also [26, Theorem 3.2]). 
Theorem 4.28. Let $\Omega$ be a complete Riemannian manifold and $H$ a separable Hilbert space. Fix $H_{0}$ as a linear subspace of $H$ and $j \in H$. Moreover, let $\left(a_{l}\right)_{l \in \mathbb{N}}$ be an orthonormal basis of $H_{0}$. Set $W=H_{0}+j$ and $Z=W \times \Omega$. Let $f: Z \rightarrow \mathbb{R}$ be a $C^{1}$ functional.

For any $m \in \mathbb{N}$, let $W_{m}=\operatorname{span}\left\{a_{1}, a_{2}, \ldots, a_{m}\right\}+j, Z_{m}=W_{m} \times \Omega$ and $f_{m}=\left.f\right|_{Z_{m}}$. Fixed $y \in \Omega$, for any $R>0$ consider the sets:

$$
\begin{aligned}
S & =\{(j, x) \in Z: x \in \Omega\}=\{j\} \times \Omega, \\
Q(R) & =\left\{(t, y) \in Z:\|t-j\|_{H} \leq R\right\},
\end{aligned}
$$

where $\|\cdot\|_{H}$ is the norm of the Hilbert space $H$.

Assume that $f_{m}$ satisfies condition $(P S)$ for any $m \in \mathbb{N}$ and there exists $R>0$ such that

$$
\sup f(Q(R))<+\infty, \quad \sup f(\partial Q(R))<\inf f(S) \text {. }
$$

Then, for any $m \in \mathbb{N}, f_{m}$ has a critical level $c_{m} \in[\inf f(S), \sup f(Q(R))]$, where

$$
\begin{gathered}
c_{m}=\inf _{h \in \Gamma_{m}} \sup _{z \in Q_{m}(R)} f_{m}(h(z)) \\
\Gamma_{m}=\left\{h \in C\left(Z_{m}, Z_{m}\right): h(z)=z \text { for all } z \in \partial Q_{m}(R)\right\}
\end{gathered}
$$

and

$$
Q_{m}(R)=\left\{(t, y) \in Z_{m}:\|t-j\|_{H} \leq R\right\}
$$

Now, we need introducing the Relative Category Theory, which generalizes the Ljusternik-Schnirelman one. To this aim, let us give the notion of relative category and some of its main properties (see, e.g., [35, 43, 94]).

Definition 4.29. Let $Y$ and $A$ be closed subsets of a topological space $Z$. The category of $A$ in $Z$ relative to $Y$, briefly cat $Z, Y(A)$, is the least integer $k$ such that there exist $k+1$ closed subsets of $Z, A_{0}, A_{1}, \ldots, A_{k}, A=A_{0} \cup A_{1} \cup \cdots \cup A_{k}$, and $k+1$ functions, $h_{l} \in C\left([0,1] \times A_{l}, Z\right), l \in\{0,1, \ldots, k\}$, such that

(a) $h_{l}(0, z)=z$ for $z \in A_{l}, 0 \leq l \leq k$;

(b) $h_{0}(1, z) \in Y$ for $z \in A_{0}$, and $h_{0}(\sigma, y) \in Y$ for all $y \in A_{0} \cap Y, \sigma \in[0,1]$;

(c) $h_{l}(1, z)=z_{l}$ for $z \in A_{l}$ and some $z_{l} \in Z, 1 \leq l \leq k$.

If a finite number of such sets does not exist, we set $\operatorname{cat}_{Z, Y}(A)=+\infty$.

We have that $\operatorname{cat}_{Z}(A)=\operatorname{cat}_{Z, \emptyset}(A)$ is the classical Ljusternik-Schnirelman category of $A$ in $Z$.

Proposition 4.30. Let $A, B, Y$ be closed subsets of a topological space $Z$.

(i) If $A \subset B$ then $\operatorname{cat}_{Z, Y}(A) \leq \operatorname{cat}_{Z, Y}(B)$;

(ii) $\operatorname{cat}_{Z, Y}(A \cup B) \leq \operatorname{cat}_{Z, Y}(A)+\operatorname{cat}_{Z}(B)$; 
(iii) if there exists $h \in C([0,1] \times A, Z)$ such that $h(\sigma, y)=y$ for $y \in A \cap Y$ and $\sigma \in[0,1]$, then $\operatorname{cat}_{Z, Y}(A) \leq \operatorname{cat}_{Z, Y}(B)$ where $B=\overline{h(1, A)}$.

Remark 4.31. Let $Z$ be a topological space and $Y$ a closed subset of $Z$. Then (ii) of Proposition 4.30 implies that the relative category and the classical LjusternikSchnirelman category are connected by the inequality

$$
\operatorname{cat}_{Z, Y}(A) \leq \operatorname{cat}_{Z}(A) \text { for any closed set } A \subset Z \text {. }
$$

It is easy to see that Definition 4.29 implies the following proposition.

Proposition 4.32. Let $Z$ be a topological space and $C, \Lambda$ be two subsets of $Z$ such that $C$ is a closed strong deformation retract of $Z \backslash \Lambda$, i.e., there exists a continuous map $\mathcal{R}:[0,1] \times(Z \backslash \Lambda) \rightarrow Z$ such that

$$
\begin{cases}\mathcal{R}(0, z)=z & \text { for all } z \in Z \backslash \Lambda, \\ \mathcal{R}(1, z) \in C & \text { for all } z \in Z \backslash \Lambda, \\ \mathcal{R}(\sigma, z)=z & \text { for all } z \in C, \sigma \in[0,1] .\end{cases}
$$

Then $\operatorname{cat}_{Z, C}(Z \backslash \Lambda)=0$.

A further property of the relative category can be stated (for the proof, see 25 Proposition 2.2]).

Proposition 4.33. Let $Y, Z^{\prime}, Y^{\prime}$ be closed subsets of a topological space $Z$ such that $Y^{\prime} \subset Z^{\prime}$. Suppose that there exist a retraction $r: Z \rightarrow Z^{\prime}$, i.e., a continuous map such that $r(z)=z$ for all $z \in Z^{\prime}$, and a homeomorphism $\Phi: Z \rightarrow Z$ such that $\Phi\left(Y^{\prime}\right) \subset Y$ and $r \circ \Phi^{-1}(Y) \subset Y^{\prime}$. Then, if $A^{\prime}$ is a closed subset of $Z^{\prime}$, it results that

$$
\operatorname{cat}_{Z, Y}\left(\Phi\left(A^{\prime}\right)\right) \geq \operatorname{cat}_{Z^{\prime}, Y^{\prime}}\left(A^{\prime}\right) .
$$

Now, we can state a multiplicity result for critical levels of a strongly indefinite functional (for the proof, see [25. Theorem 1.4]).

Theorem 4.34. Let $Z$ be a $C^{2}$ complete Riemannian manifold modelled on a Hilbert space and $f: Z \rightarrow \mathbb{R}$ a $C^{1}$ functional. Let us assume that there exist two subsets $\Lambda$ and $C$ of $Z$ such that $C$ is a closed strong deformation retract of $Z \backslash \Lambda$,

$$
\inf _{z \in \Lambda} f(z)>\sup _{z \in C} f(z) \quad \text { and } \operatorname{cat}_{Z, C}(Z)>0 .
$$

If $f$ satisfies condition $(P S)$, then it has at least cat $_{Z, C}(Z)$ critical points in $Z$ whose critical levels are greater or equal than $\inf f(\Lambda)$. Moreover, if $\operatorname{cat}_{Z, C}(Z)=$ $+\infty$, there exists a sequence $\left(z_{k}\right)_{k}$ of critical points of $f$ such that

$$
\lim _{k \rightarrow+\infty} f\left(z_{k}\right)=\sup _{z \in Z} f(z) .
$$

Remark 4.35. In Theorem 4.34 the critical levels $c_{k}$ are characterized as follows:

$$
c_{k}=\inf _{B \in F_{k}} \sup _{z \in B} f(z) \quad \text { for any } k \in \mathbb{N}, 1 \leq k \leq \operatorname{cat}_{Z, C}(Z),
$$

where

$$
F_{k}=\left\{B \subset Z: B \text { closed, } \operatorname{cat}_{Z, C}(B) \geq k\right\} .
$$


The aim of this section is to investigate the existence of geodesics joining two fixed points in a Lorentzian manifold which splits in a "good" product but it has a metric depending on both its coordinates. The simplest model of this type can be defined as follows.

Definition 4.36. A Lorentzian manifold $\left(M,\langle\cdot, \cdot\rangle_{L}\right)$ is an orthogonal splitting spacetime if it is $M=\mathbb{R} \times M_{0}$ and

$$
\left\langle\zeta, \zeta^{\prime}\right\rangle_{L}=-\beta(z) \tau \tau^{\prime}+\left\langle\alpha(z) \xi, \xi^{\prime}\right\rangle_{R},
$$

for any $z=(t, x) \in M\left(t \in \mathbb{R}, x \in M_{0}\right)$, and $\zeta=(\tau, \xi), \zeta^{\prime}=\left(\tau^{\prime}, \xi^{\prime}\right) \in T_{z} M \equiv \mathbb{R} \times$ $T_{x} M_{0}$, where $\left(M_{0},\langle\cdot, \cdot\rangle_{R}\right)$ is a finite dimensional connected Riemannian manifold, $\alpha(z)$ is a smooth symmetric linear strictly positive operator from $T_{x} M_{0}$ into itself and $\beta: M \rightarrow \mathbb{R}$ is a smooth and strictly positive scalar field.

Clearly, if both $\alpha$ and $\beta$ do not depend on $t$, Definition 4.36 reduces to the static metric (4.8) and the geodesic connectedness result is Theorem 4.15 already proved with a quadratic growth assumption on $\beta$ (once that $\alpha$ is "uniformly" far away from zero). But, in general, when coefficients depend on time component $t$, stronger assumptions, in particular a "good" control on the partial derivatives $\alpha_{t}$, $\beta_{t}$, need. More precisely, the following result can be stated.

Theorem 4.37. Let $M=\mathbb{R} \times M_{0}$ be an orthogonal splitting manifold such that $\left(M_{0},\langle\cdot, \cdot\rangle_{R}\right)$ is a complete Riemannian manifold. Assume that there exist some constants $\lambda, \nu, N, K>0$ such that the coefficients $\alpha, \beta$ of its metric 4.13) satisfy the following hypotheses:

$$
\begin{gathered}
\lambda\langle\xi, \xi\rangle_{R} \leq\langle\alpha(z) \xi, \xi\rangle_{R} ; \\
\nu \leq \beta(z) \leq N ; \\
\left|\beta_{t}(z)\right| \leq K, \quad\left|\left\langle\alpha_{t}(z) \xi, \xi\right\rangle_{R}\right| \leq K\langle\xi, \xi\rangle_{R} ;
\end{gathered}
$$

for all $z=(t, x) \in M, \xi \in T_{x} M_{0}$. Furthermore, assume that

$$
\begin{gathered}
\limsup _{t \rightarrow+\infty}\left(\sup \left\{\left\langle\alpha_{t}(t, x) \xi, \xi\right\rangle_{R}: x \in M_{0}, \xi \in T_{x} M_{0},\langle\xi, \xi\rangle_{R}=1\right\}\right) \leq 0 \\
\liminf _{t \rightarrow-\infty}\left(\inf \left\{\left\langle\alpha_{t}(t, x) \xi, \xi\right\rangle_{R}: x \in M_{0}, \xi \in T_{x} M_{0},\langle\xi, \xi\rangle_{R}=1\right\}\right) \geq 0 .
\end{gathered}
$$

Then, fixed $p=\left(t_{p}, x_{p}\right), q=\left(t_{q}, x_{q}\right) \in M$, there exists at least one geodesic joining $p$ to $q$ which is surely non-trivial if $p \neq q$.

Moreover, if $M_{0}$ is not contractible in itself and there exists $\lambda_{1}>0$ such that

$$
\langle\alpha(0, x) \xi, \xi\rangle_{R} \leq \lambda_{1}\langle\xi, \xi\rangle_{R} \quad \text { for all } x \in M_{0}, \xi \in T_{x} M_{0},
$$

infinitely many such (spacelike) geodesics $\left(z_{k}\right)_{k}$ exist so that $f\left(z_{k}\right) \nearrow+\infty$. 
The first problem in applying directly the abstract theorems to action functional $f$ in (1.1), is that $f$ may not satisfy condition $(P S)$ on the whole manifold $\Omega^{1}(p, q ; M)$. Furthermore, a finite dimensional decomposition of $W^{1}\left(t_{p}, t_{q}\right)$ needs in order to apply not only Theorem 4.28 obvious for its statement, but also Theorem 4.34 as we have to be sure that we deal with manifolds with non-trivial relative category.

A way to overcome the lack of condition $(P S)$ is to use a penalization argument on $f$ as follows.

For any $\varepsilon>0$, let $\psi_{\varepsilon}: \mathbb{R}_{+} \rightarrow \mathbb{R}_{+}$be the "cut-function" defined as

$$
\psi_{\varepsilon}(s)= \begin{cases}0 & \text { if } 0 \leq s \leq \frac{1}{\varepsilon} \\ \sum_{n=3}^{+\infty} \frac{\left(s-\frac{1}{\varepsilon}\right)^{n}}{n !} & \text { if } s>\frac{1}{\varepsilon} .\end{cases}
$$

Obviously, $\psi_{\varepsilon}$ is a $C^{2}$ map on $\mathbb{R}_{+}$, it is increasing and there exist two positive constants, $a, b$, such that

$$
\psi_{\varepsilon}^{\prime}(s) \geq \psi_{\varepsilon}(s) \geq a s-b, \quad s \psi_{\varepsilon}^{\prime}(s) \geq \psi_{\varepsilon}(s) \text { for all } s \in \mathbb{R}_{+} .
$$

Thus, for any $\varepsilon>0$ we consider the perturbed functional

$$
\begin{aligned}
f_{\varepsilon}(z) & =f(z)-\psi_{\varepsilon}\left(\left\|t^{\prime}\right\|^{2}\right) \\
& =\int_{0}^{1}\left(\left\langle\alpha(t, x) x^{\prime}, x^{\prime}\right\rangle_{R}-\beta(t, x)\left(t^{\prime}\right)^{2}\right) d s-\psi_{\varepsilon}\left(\left\|t^{\prime}\right\|^{2}\right)
\end{aligned}
$$

for any $z=(t, x) \in \Omega^{1}(p, q ; M)$, where $\left\|t^{\prime}\right\|^{2}=\int_{0}^{1}\left|t^{\prime}\right|^{2} d s$. Clearly, it is

$$
f_{\varepsilon}(z) \leq f(z) \quad \text { for all } z \in \Omega^{1}(p, q ; M),
$$

furthermore, the Fréchet differential of $f_{\varepsilon}$ at $z=(t, x) \in \Omega^{1}(p, q ; M)$ is given by

$$
f_{\varepsilon}^{\prime}(z)[\zeta]=2 \int_{0}^{1}\left\langle z^{\prime}, \frac{D \zeta}{d s}\right\rangle_{L} d s-2 \psi_{\varepsilon}^{\prime}\left(\left\|t^{\prime}\right\|^{2}\right) \int_{0}^{1} t^{\prime} \tau^{\prime} d s
$$

for all $\zeta=(\tau, \xi) \in T_{z} \Omega^{1}(p, q ; M)$.

Taken any $z_{\varepsilon}=\left(t_{\varepsilon}, x_{\varepsilon}\right)$ critical point of $f_{\varepsilon}$, some usual bootstrap arguments allow one to prove that it is smooth and satisfies the equation

$$
\frac{D z^{\prime}}{d s}-\psi_{\varepsilon}^{\prime}\left(\left\|t^{\prime}\right\|^{2}\right)\left(t^{\prime \prime}, 0\right)=0 ;
$$

hence, a constant $E_{\varepsilon}\left(z_{\varepsilon}\right) \in \mathbb{R}$ exists so that

$$
\left\langle z_{\varepsilon}^{\prime}(s), z_{\varepsilon}^{\prime}(s)\right\rangle_{L}-\psi_{\varepsilon}^{\prime}\left(\left\|t_{\varepsilon}^{\prime}\right\|^{2}\right)\left(t_{\varepsilon}^{\prime}\right)^{2}(s) \equiv E_{\varepsilon}\left(z_{\varepsilon}\right) \quad \text { for all } s \in I .
$$

Thus, by integrating (4.23), according to (4.21) it follows that

$$
E_{\varepsilon}\left(z_{\varepsilon}\right)=f\left(z_{\varepsilon}\right)-\psi_{\varepsilon}^{\prime}\left(\left\|t_{\varepsilon}^{\prime}\right\|^{2}\right)\left\|t_{\varepsilon}^{\prime}\right\|^{2} \leq f_{\varepsilon}\left(z_{\varepsilon}\right) .
$$


Obviously, if $L^{*}>0$ is a fixed constant, by (4.20) and (4.22) it follows that a critical point $z_{\varepsilon}=\left(t_{\varepsilon}, x_{\varepsilon}\right)$ of the penalized functional $f_{\varepsilon}$ is also a critical point of the action functional $f$ if it is such that

$$
\left\|t_{\varepsilon}^{\prime}\right\|^{2} \leq L^{*} \quad \text { and } \quad \varepsilon \leq \frac{1}{L^{*}} .
$$

Remark 4.38. A direct consequence of the given hypotheses is that on sublevels of $f$ the norm of the $t$ component "controls" the $x$ component. In fact, taken $z=(t, x)$ such that $f_{\varepsilon}(z) \leq L, L \in \mathbb{R}$, then (4.14), (4.15) and (4.22) imply that

$$
\lambda \int_{0}^{1}\left\langle x^{\prime}, x^{\prime}\right\rangle_{R} d s \leq \int_{0}^{1}\left\langle\alpha(z) x^{\prime}, x^{\prime}\right\rangle_{R} d s \leq L+N\left\|t^{\prime}\right\|^{2}+\psi_{\varepsilon}\left(\left\|t^{\prime}\right\|^{2}\right) .
$$

Lemma 4.39. If $M_{0}$ is complete and 4.14), 4.15), 4.16) hold, then taken any $\varepsilon>0$ the corresponding functional $f_{\varepsilon}$ satisfies condition $(P S)$ on $\Omega^{1}(p, q ; M)$.

Proof. Up to small changes, the main ideas of this proof can be found in [26] Proposition 4.4]. Here, for completeness, we outline the main steps.

Let $L \in \mathbb{R}$ and $\left(z_{k}\right)_{k} \subset \Omega^{1}(p, q ; M)$ be such that

$$
f_{\varepsilon}\left(z_{k}\right) \leq L \text { for all } k \in \mathbb{N}, \quad f_{\varepsilon}^{\prime}\left(z_{k}\right) \rightarrow 0 \text { if } k \rightarrow+\infty .
$$

Firstly, suitable computations making use of hypotheses (4.14), 4.15), (4.16) and properties on $\psi_{\varepsilon}$ in (4.21), allow one to prove that $\left(\left\|t_{k}^{\prime}\right\|\right)_{k}$ is bounded; then, by Remark 4.38 also $\left(\left\|x_{k}^{\prime}\right\|\right)_{k}$ is bounded. Hence, $\left(z_{k}\right)_{k}$ is a bounded sequence in $\Omega^{1}(p, q ; M)$.

Thus, since $M_{0}$ is complete, by means of Lemma 4.7 and careful limit estimates, there exists $z \in \Omega^{1}(p, q ; M)$ such that $z_{k} \rightarrow z$ strongly in $\Omega^{1}(p, q ; M)$ (up to subsequences).

Now, once we are able to find critical points of some $f_{\varepsilon}$, the problem is to "reduce" them to critical points of the "original" functional $f$ by means of some a priori estimates as 4.25).

Lemma 4.40. If the hypotheses 4.14) - 4.18) hold, then for each fixed $L>0$ there exists $\varepsilon_{0}>0$ such that, if $z_{\varepsilon}=\left(t_{\varepsilon}, x_{\varepsilon}\right) \in \Omega^{1}(p, q ; M)$ is such that

$$
f_{\varepsilon}^{\prime}\left(z_{\varepsilon}\right)=0 \quad \text { and } \quad f_{\varepsilon}\left(z_{\varepsilon}\right) \leq L,
$$

with $\varepsilon \leq \varepsilon_{0}$, then it is $\left\|t_{\varepsilon}^{\prime}\right\|^{2} \leq \frac{1}{\varepsilon_{0}}$; hence, $\psi_{\varepsilon}\left(\left\|t_{\varepsilon}^{\prime}\right\|^{2}\right)=0$.

Proof. Also for this proof, the main ideas are in [26] Propositions 5.2 and 5.3] but, anyway, here we outline the main steps.

Firstly, we want to prove that there exists $L^{*}>0$, independent of $\varepsilon$, such that if (4.26) holds then $\left|t_{\varepsilon}\right|_{\infty} \leq L^{*}$. To this aim, taken any $\mu>0$ and by means of hypotheses (4.17), (4.18), there exists $t_{\mu}>\max \left\{\left|t_{p}\right|,\left|t_{q}\right|\right\}$ such that, by using (4.14), for all $x \in M_{0}, \xi \in T_{x} M_{0}$ it is

$$
\begin{array}{cl}
\left\langle\alpha_{t}(t, x) \xi, \xi\right\rangle_{R} \leq \lambda \mu\langle\xi, \xi\rangle_{R} \leq \mu\langle\alpha(t, x) \xi, \xi\rangle_{R} & \text { if } t \geq t_{\mu}, \\
\left\langle\alpha_{t}(t, x) \xi, \xi\right\rangle_{R} \geq-\lambda \mu\langle\xi, \xi\rangle_{R} \geq-\mu\langle\alpha(t, x) \xi, \xi\rangle_{R} & \text { if } t \leq-t_{\mu}
\end{array}
$$


Assume that $z_{\varepsilon}=\left(t_{\varepsilon}, x_{\varepsilon}\right)$ exists such that (4.26) holds and $\left\|t_{\varepsilon}\right\|_{\infty}>t_{\mu}$, as, for example, it is $\operatorname{ess} \sup t_{\varepsilon}(s)>t_{\mu}$. Hence, an interval $\left.[a, b] \subset\right] 0,1[$ exists such that $t_{\varepsilon}(s)>t_{\mu}$ for all $\left.s \in I\right] a, b\left[, t_{\varepsilon}(a)=t_{\varepsilon}(b)=t_{\mu}\right.$. Fixed $\omega>0$, let us define function $\tau: I \rightarrow \mathbb{R}$ such that

$$
\tau(s)= \begin{cases}\sinh \left(\omega\left(t_{\varepsilon}(s)-t_{\mu}\right)\right) & \text { if } s \in[a, b] \\ 0 & \text { if } s \in I \backslash[a, b] .\end{cases}
$$

Clearly, it is $\tau \in H_{0}^{1}(I, \mathbb{R})$; thus, it is $(\tau, 0) \in T_{z_{\varepsilon}} \Omega^{1}(p, q ; M)$ and $f_{\varepsilon}^{\prime}\left(z_{\varepsilon}\right)[(\tau, 0)]=0$. By means of careful estimates, the main properties of function $y=\sinh \sigma$, (4.14)(4.16) and (4.24) with (4.26), suitable choices of $\mu$ and $\omega$ allow one to prove that there exists $\theta>0$ (depending on $\mu$ but independent of $\varepsilon$ ) such that $\omega \sqrt{\theta}<2$ and

$$
\int_{a}^{b}\left(t_{\varepsilon}^{\prime}\right)^{2} \cosh \left(\omega\left(t_{\varepsilon}-t_{\mu}\right)\right) d s \leq \theta \int_{a}^{b} \cosh \left(\omega\left(t_{\varepsilon}-t_{\mu}\right)\right) d s .
$$

Hence, by applying [48, Lemma 3.4], a constant $L^{*}>0$ exists, independent of $\varepsilon$, such that it has to be ess $\sup _{s \in I} t_{\varepsilon}(s) \leq L^{*}$. Similarly, one can argue if it is $\operatorname{ess} \sup _{s \in I}\left(-t_{\varepsilon}(s)\right)>t_{\mu}$. Hence, in any case it has to be $\left|t_{\varepsilon}\right|_{\infty} \leq L^{*}$.

Now, we have to prove that also $\left\|t_{\varepsilon}^{\prime}\right\|$ is bounded independently of $\varepsilon$ if (4.26) holds. For this proof, let us start from $f_{\varepsilon}^{\prime}\left(z_{\varepsilon}\right)[(\tau, 0)]=0$ with

$$
\tau(s)=\sinh \left(\omega\left(t_{\varepsilon}(s)-j^{*}(s)\right), \quad s \in I,\right.
$$

with $\omega>0$ any, up to now. By making use of "good" tricks as Young inequality

$\int_{0}^{1}\left|t^{\prime}\right| \cosh \left(\omega\left(t_{\varepsilon}-j^{*}\right)\right) d s \leq \frac{\delta}{2} \int_{0}^{1}\left(t^{\prime}\right)^{2} \cosh \left(\omega\left(t_{\varepsilon}-j^{*}\right)\right) d s+\frac{1}{2 \delta} \int_{0}^{1} \cosh \left(\omega\left(t_{\varepsilon}-j^{*}\right)\right) d s$,

for any $\delta>0$, and of estimates due to the boundedness of $\left|t_{\varepsilon}\right|_{\infty}$ such as

$$
1 \leq \cosh \left(\omega\left(t_{\varepsilon}(s)-j^{*}(s)\right)\right) \leq \gamma \quad \text { for all } s \in I,
$$

( $\gamma$ independent of $\varepsilon$ ), and by taking into account the given hypotheses (4.14) - 4.16) and by doing straightforward calculations, a suitable choice of a large enough $\omega$ and a small enough $\delta$ (so to have strictly positive all the coefficients in the inequality below), allow one to prove that

$$
\begin{aligned}
& \left(2 \omega \nu-\frac{K N}{\lambda}-K-\omega\left|\Delta_{t}\right| N \delta\right)\left\|t^{\prime}\right\|^{2}+\left(2 \omega-\frac{K}{\lambda}\right) \psi_{\varepsilon}^{\prime}\left(\left\|t^{\prime}\right\|^{2}\right)\left\|t^{\prime}\right\|^{2} \\
& \leq \gamma\left(\frac{K L}{\lambda}+2 \omega \Delta_{t}^{2} \psi_{\varepsilon}^{\prime}\left(\left\|t^{\prime}\right\|^{2}\right)+N\left|\Delta_{t}\right| \frac{\omega}{\delta}\right) .
\end{aligned}
$$

Hence, the proof follows from 4.21).

Thus, from now on in this section, let us assume that the hypotheses of Theorem 4.37 are satisfied and, as a further step, let us introduce a finite dimensional decomposition of $W^{1}\left(t_{p}, t_{q}\right)$. 
So, let us consider the orthonormal basis $\{\sin (l \pi s)\}_{l \in \mathbb{N}}$ of $H_{0}^{1}(I, \mathbb{R})$ and for any $m \in \mathbb{N}$ define

$$
W_{m}=H_{m}+j^{*}, \quad \text { with } H_{m}=\operatorname{span}\{\sin (l \pi s): l \in\{1,2, \ldots, m\}\},
$$

and

$$
Z_{m}=W_{m} \times \Omega^{1}\left(x_{p}, x_{q} ; M_{0}\right), \quad f_{\varepsilon, m}=\left.f_{\varepsilon}\right|_{Z_{m}}(\text { for any } \varepsilon>0) .
$$

Clearly, by arguing as in Proposition 4.39 the following lemma can be stated.

Lemma 4.41. For any $\varepsilon>0$ and $m \in \mathbb{N}$, the functional $f_{\varepsilon, m}$ satisfies condition $(P S)$.

Obviously, fixed $\varepsilon>0$, once we are able to find critical points of $f_{\varepsilon, m}$ on $Z_{m}$, it is necessary to come back to the critical points of $f_{\varepsilon}$ on the whole manifold $\Omega^{1}(p, q ; M)$. Thus, to this aim, we need the following proposition.

Lemma 4.42. Assume that for all $m \in \mathbb{N}$ there exists $z_{m} \in Z_{m}$ critical point of $f_{\varepsilon, m}$ on $Z_{m}$ such that

$$
c_{1} \leq f_{\varepsilon, m}\left(z_{m}\right) \leq c_{2}
$$

for two given constants $c_{1}$ and $c_{2}$ (independent of $m$ ). Then, sequence $\left(z_{m}\right)_{m \in \mathbb{N}}$ converges, up to a subsequence, to a critical point $z \in \Omega^{1}(p, q ; M)$ of $f_{\varepsilon}$ such that

$$
c_{1} \leq f_{\varepsilon}(z) \leq c_{2} .
$$

Proof. By arguing as in the proof of Proposition 4.39 it follows that $\left(z_{m}\right)_{m \in \mathbb{N}}$ is a bounded sequence of $\Omega^{1}(p, q ; M)$; hence, up to a subsequence, $z \in \Omega^{1}(p, q ; M)$ exists so that $z_{m} \rightarrow z$ weakly in $\Omega^{1}(p, q ; M)$.

Set $z_{m}=\left(t_{m}, x_{m}\right), z=(t, x)$, from Lemma 4.7 it follows that two sequences $\xi_{m} \in T_{x_{m}} \Omega^{1}\left(x_{p}, x_{q} ; M_{0}\right)$ and $\nu_{m} \in H^{1}\left(I, \mathbb{R}^{N}\right)$ exist such that (4.6) holds. Thus, denoted by $P_{m}: H_{0}^{1}(I, \mathbb{R}) \rightarrow H_{m}$ the orthogonal projection between such two spaces and $\tau_{m}=P_{m}\left(t-j^{*}\right)-\left(t_{m}-j^{*}\right) \in H_{m} \equiv T_{t_{m}} W_{m}$, it is $f_{\varepsilon, m}^{\prime}\left(z_{m}\right)\left[\left(\tau_{m}, \xi_{m}\right)\right]=0$ for all $m \in \mathbb{N}$. Whence, the same arguments of the second part of the proof of Proposition 4.39] show that $z_{m} \rightarrow z$ strongly in $\Omega^{1}(p, q ; M)$ while from [13, Lemma 3.4 ] it follows that $f_{\varepsilon}^{\prime}(z)=0$ and (4.27) holds.

Now, we want to apply Theorem 4.28 to functional $f_{\varepsilon}$ in $\Omega^{1}(p, q ; M)$. Fixed $y \in \Omega^{1}\left(x_{p}, x_{q} ; M_{0}\right)$ so that it is a $C^{1}$ function, let us define the sets:

$$
\begin{aligned}
S & =\left\{j^{*}\right\} \times \Omega^{1}\left(x_{p}, x_{q} ; M_{0}\right), \\
Q(R) & =\left\{(t, y) \in \Omega^{1}(p, q ; M):\left\|t^{\prime}-\Delta_{t}\right\| \leq R\right\} \quad(\text { for any } R>0),
\end{aligned}
$$

being $\frac{d j^{*}}{d s}(s) \equiv \Delta_{t}$. Obviously, taken $\left.\left.\varepsilon_{0} \in\right] 0,1\right]$ so that $\Delta_{t}^{2}<\frac{1}{\varepsilon_{0}}$, from (4.20) it follows

$$
\left.\left.\psi_{\varepsilon}\left(\left\|\Delta_{t}\right\|^{2}\right)=0 \quad \text { for all } \varepsilon \in\right] 0, \varepsilon_{0}\right] ;
$$

hence, (4.14) and (4.15) imply

$$
\left.\left.\inf f_{\varepsilon}(S) \geq-N \Delta_{t}^{2} \quad \text { for all } \varepsilon \in\right] 0, \varepsilon_{0}\right] .
$$


On the other hand, by means of suitable computations (for more details, see the proof of [26] Theorem 1.4]), two positive constants $\bar{k}_{1}$ and $\bar{k}_{2}$ exist, independent of $\varepsilon$ and $\Delta_{t}$, such that for all $z \in Q(R)$ it is

$$
f_{\varepsilon}(z) \leq f(z) \leq \bar{k}_{1}+\bar{k}_{2}\left\|t^{\prime}\right\|-\nu\left\|t^{\prime}\right\|^{2} \leq \bar{k}_{1}+\bar{k}_{2} \sqrt{R^{2}+\Delta_{t}^{2}}-\nu\left(R^{2}+\Delta_{t}^{2}\right) .
$$

So, not only it is

$$
\sup f_{\varepsilon}(Q(R))<+\infty \quad \text { for all } R>0
$$

but also there exists $R$ large enough so that

$$
\sup f_{\varepsilon}(\partial Q(R))<\inf f_{\varepsilon}(S) .
$$

Thus, the existence of a critical point for the action functional $f$ in $\Omega^{1}(p, q ; M)$, i.e., a geodesic joining $p$ to $q$ in $M$, follows from Theorem 4.28 and Lemmas 4.40 4.414 .42

Now, in order to apply Theorem 4.34 it is necessary exploiting the topological properties of the space of curves $\Omega^{1}(p, q ; M)$. To this aim, the following result is basic and extends Proposition 4.8 to the relative category of a product set involving $\Omega^{1}\left(x_{p}, x_{q} ; M_{0}\right)$ (for more details, see [37] Corollary 3.2]).

Proposition 4.43. Let $M_{0}$ be a simply connected and non-contractible smooth manifold, $x_{p}$ and $x_{q}$ two points of $M_{0}$ and $D^{m}$ the unit disk in $\mathbb{R}^{m}$ with boundary $S^{m}$. Then, for any $k \in \mathbb{N}$, there exists a compact subset $V_{m, k}$ of $D^{m} \times$ $\Omega^{1}\left(x_{p}, x_{q} ; M_{0}\right)$ such that

$$
\text { cat }_{D^{m} \times \Omega^{1}\left(x_{p}, x_{q} ; M_{0}\right), S^{m} \times \Omega^{1}\left(x_{p}, x_{q} ; M_{0}\right)}\left(V_{m, k}\right) \geq k .
$$

Remark 4.44. Let $\left(V_{m, k}\right)_{m, k}$ be the family of compact subsets of the product manifold $D^{m} \times \Omega^{1}\left(x_{p}, x_{q} ; M_{0}\right)$ obtained in Proposition 4.43 Fixed $k \in \mathbb{N}$, by means of [37. Proposition 2.12, Theorems 2.14 and 3.1] it follows that, for each $m \in \mathbb{N}$ the set $V_{m, k}$ has the same projection on $\Omega^{1}\left(x_{p}, x_{q} ; M_{0}\right)$, i.e., there exists $V_{k}^{x}$ compact subset of $\Omega^{1}\left(x_{p}, x_{q} ; M_{0}\right)$, independent on $m$, and $V_{m, k}^{t} \subset D^{m}$ such that $V_{m, k}=V_{m, k}^{t} \times V_{k}^{x}$.

Lemma 4.45. There exists a continuous map $\varrho: \mathbb{R}_{+} \rightarrow \mathbb{R}_{+}$such that

$z=(t, x) \in \Omega^{1}(p, q ; M),\left\|t^{\prime}-\Delta_{t}\right\|=\varrho\left(\left\|x^{\prime}\right\|\right) \quad \Longrightarrow \quad f(z) \leq \begin{cases}-2 N \Delta_{t}^{2} & \text { if } \Delta_{t} \neq 0, \\ -1 & \text { if } \Delta_{t}=0,\end{cases}$

where $N$ is defined as in 4.15.).

Proof. Taken $z=(t, x) \in \Omega^{1}(p, q ; M)$, then (4.15), (4.16) and assumption (4.19) allow one to prove that

$$
f(z) \leq\left(\lambda_{1}+K\left|\Delta_{t}\right|\right)\left\|x^{\prime}\right\|^{2}+K\left\|x^{\prime}\right\|^{2}\left\|t^{\prime}-\Delta_{t}\right\|-\nu\left\|t^{\prime}-\Delta_{t}\right\|^{2} .
$$

Hence, it is enough to define

$$
\varrho(r)=\frac{K r^{2}}{2 \nu}+\sqrt{\frac{K^{2} r^{4}}{4 \nu^{2}}+\frac{\left(\lambda_{1}+K\left|\Delta_{t}\right|\right) r^{2}+2 N \Delta_{t}^{2}}{\nu}} .
$$


Now, fixed $m \in \mathbb{N}$, let us define the "cylinder"

$$
C_{m}=\left\{z=(t, x) \in Z_{m}:\left\|t^{\prime}-\Delta_{t}\right\|=\varrho\left(\left\|x^{\prime}\right\|\right)\right\} .
$$

For each $\varepsilon>0$, it is not difficult to show that (4.20), 4.29) and Lemma 4.45) imply

$$
\sup f_{\varepsilon, m}\left(C_{m}\right)<\inf f_{\varepsilon, m}(S)
$$

being $S$ as in (4.28). Furthermore, the subset $C_{m}$ is a strong deformation retract of $Z_{m} \backslash S$; hence, from Proposition 4.32 it follows cat $Z_{m}, C_{m}\left(Z_{m} \backslash S\right)=0$.

Lemma 4.46. Let $M_{0}$ be 1-connected. For any $m, k \in \mathbb{N}$ there exists a compact subset $K_{m, k}$ of $Z_{m}$, whose projection on $\Omega^{1}\left(x_{p}, x_{q} ; M_{0}\right)$ is independent of $m$, such that

$$
\operatorname{cat}_{Z_{m}, C_{m}}\left(K_{m, k}\right) \geq k \text {. }
$$

Proof. Considered the following sets:

$$
\begin{gathered}
D_{m}=\left\{t \in W_{m}:\left\|t^{\prime}-\Delta_{t}\right\| \leq 1\right\}, \quad \tilde{D}_{m}=D_{m} \times \Omega^{1}\left(x_{p}, x_{q} ; M_{0}\right), \\
\Sigma_{m}=\partial D_{m}=\left\{t \in W_{m}:\left\|t^{\prime}-\Delta_{t}\right\|=1\right\}, \quad \tilde{\Sigma}_{m}=\Sigma_{m} \times \Omega^{1}\left(x_{p}, x_{q} ; M_{0}\right),
\end{gathered}
$$

by Proposition 4.43 there exists a compact set $V_{m, k}$ in $\tilde{B}_{m}$ such that

$$
\operatorname{cat}_{\tilde{B}_{m}, \tilde{\Sigma}_{m}}\left(V_{m, k}\right) \geq k \text {. }
$$

Furthermore, we can construct a retraction from $Z_{m}$ onto $\tilde{B}_{m}$, and an homeomorphism from $Z_{m}$ onto itself so that Proposition 4.33 applies and the proof follows from Remark 4.44 (for more details, see [26. Lemma 7.4]).

Assumed that $M_{0}$ is a 1-connected manifold (it can be done without loss of generality) and $\varepsilon$ small enough, from Lemmas 4.41 and 4.46 it follows that Theorem 4.34 can be applied to each functional $f_{\varepsilon, m}$ in $Z_{m}$. Hence, there exists a sequence of critical points $\left(z_{\varepsilon, m}^{k}\right)_{k} \subset Z_{m}$ of $f_{\varepsilon, m}$ such that

$$
f_{\varepsilon, m}\left(z_{\varepsilon, m}^{k}\right) \geq \inf f_{\varepsilon, m}(S), \quad \lim _{k \rightarrow+\infty} f_{\varepsilon, m}\left(z_{\varepsilon, m}^{k}\right)=\sup f_{\varepsilon, m}\left(Z_{m}\right)=+\infty .
$$

Moreover, carefull estimates allow one to prove that for each $k \geq 1$ there exists a constant $\gamma_{k}>0$, independent of $\varepsilon$ and $m$, such that

$$
f_{\varepsilon, m}\left(z_{\varepsilon, m}^{k}\right) \leq \gamma_{m}
$$

while for each $c>0$ there exists $k_{c} \in \mathbb{N}$, independent of $\varepsilon$ and $m$, such that

$$
f_{\varepsilon, m}\left(z_{\varepsilon, m}^{k}\right) \geq \lambda c-\Delta_{t}^{2} N \quad \text { for all } k \geq k_{c}
$$

(being $\lambda, N$ as in the given hypotheses).

Whence, Lemmas 4.40 and 4.42 imply that for any $k \geq k_{c}$ there exists a critical point $z^{k} \in \Omega^{1}(p, q ; M)$ of $f$ such that

$$
\lambda c-\Delta_{t}^{2} N \leq f\left(z^{k}\right) \leq \gamma_{k}
$$

and the end of the proof of Theorem 4.37 follows from the arbitrariness of $c>0$. 
Remark 4.47. To our knowledge, up to now no better result of geodesic connectedness has been obtained in the orthogonal splitting case. We emphasize that the assumptions in Theorem 4.37 imply global hyperbolicity [82. Even though every globally hyperbolic spacetime is orthogonal splitting ([18], see also [63]), it may not satisfy assumptions in Theorem 4.37 (as there are non-geodesically connected counterexamples, including those in the static case). So, following the careful approaches introduced in the stationary case, it should be interesting to obtain a result similar to that one of Theorem 4.37 also under weaker assumptions on the metric or under intrisinsic hypotheses more related to the geometry of the manifold.

\section{References}

[1] Alías, L.J., Romero, A., Sánchez, M., Uniqueness of complete spacelike hypersurfaces of constant mean curvature in generalized Robertson-Walker spacetimes, Gen. Relativity Gravitation 27 (1995), 71-84.

[2] Ambrosetti, A., Critical Points and Nonlinear Variational Problems, Mém. Soc. Math. France (N.S.) 49, 1992.

[3] Bartolo, R., Critical Curves on Riemannian and Lorentzian Manifolds with Boundary, PhD Thesis, Departamento de Geometría y Topología, Universidad de Granada, Granada, 2000.

[4] Bartolo, R., Candela, A.M., Flores, J.L., Geodesic connectedness of stationary spacetimes with optimal growth, J. Geom. Phys. 56 (2006), 2025-2038.

[5] Bartolo, R., Candela, A.M., Flores, J.L., Sánchez, M., Geodesics in static Lorentzian manifolds with critical quadratic behavior, Adv. Nonlinear Stud. 3 (2003), 471-494.

[6] Bartolo, R., Germinario, V., Sánchez, M., Periodic trajectories with fixed energy on Riemannian and Lorentzian manifolds with boundary, Ann. Mat. Pura Appl. CLXXVII (1999), 241-262.

[7] Bartolo, R., Germinario, V., Sánchez, M., Convexity of domains of Riemannian manifolds, Ann. Global Anal. Geom. 21 (2002), 63-84.

[8] Bartolo, R., Germinario, V., Sánchez, M., Existence of a closed geodesic on noncompact Riemannian manifolds with boundary, Adv. Nonlinear Stud. 2 (2002), 51-70.

[9] Beem, J.K., Stability of geodesic incompleteness, Contemporary Math. 170 (1994), $1-12$.

[10] Beem, J.K., Ehrlich, P.E., Geodesic completeness and stability, Math. Proc. Cambridge Philos. Soc. 102 (1987), 319-328.

[11] Beem, J.K., Ehrlich, P.E., Easley, K.L., Global Lorentzian Geometry, Monographs Textbooks Pure Appl. Math. 202, Dekker Inc., New York, 1996.

[12] Benci, V., Fortunato, D., Periodic trajectories for the Lorentz metric of a static gravitational field. In: Variational Methods (H. Berestycki, J.M. Coron, I. Ekeland Eds), Birkhäuser, Basel (1990), 413-429.

[13] Benci, V., Fortunato, D., On the existence of infinitely many geodesics on space-time manifolds, Adv. Math. 105 (1994), 1-25. 
[14] Benci, V., Fortunato, D., Giannoni, F., On the existence of multiple geodesics in static space-times, Ann. Inst. H. Poincaré Anal. Non Linéaire 8 (1991), 79-102.

[15] Benci, V., Fortunato, D., Masiello, A., On the geodesic connectedness of Lorentzian manifolds, Math. Z. 217 (1994), 73-93.

[16] Benci, V., Giannoni, F., On the existence of closed geodesics on noncompact Riemannian manifolds, Duke Math. J. 68 (1992), 195-215.

[17] Bernal, A.N., Sánchez, M., On smooth Cauchy hypersurfaces and Geroch's splitting theorem, Commun. Math. Phys. 243 (2003), 461-470.

[18] Bernal, A. N. and Sánchez, M.: Smoothness of time functions and the metric splitting of globally hyperbolic spacetimes. Commun. Math. Phys. 257, 43-50 (2005).

[19] Calabi, E., Markus, L., Relativistic space forms, Ann. of Math. (2) 75 (1962), 63-76.

[20] Candela, A.M., Periodic trajectories in Lorentzian manifolds, Nonlinear Anal. TMA 30 (1997), 579-587.

[21] Candela, A.M., Multiplicity of timelike geodesics in splitting Lorentzian manifolds. In: Recent Developments in General Relativity (B. Casciaro, D. Fortunato, M. Francaviglia, A. Masiello Eds), Springer, Milano (2000), 299-306.

[22] Candela, A.M., Flores, J.L., Sánchez, M., A quadratic Bolza-type problem in a Riemannian manifold, J. Differential Equations 193 (2003), 196-211.

[23] Candela, A.M., Flores, J.L., Sánchez, M., On general Plane Fronted Waves. Geodesics, Gen. Relativity Gravitation 35 (2003), 631-649.

[24] Candela, A.M., Flores, J.L., Sánchez, M., Global hyperbolicity and Palais-Smale condition for action functionals in stationary spacetimes. Preprint ESI 2006.

[25] Candela, A.M., Giannoni, F., Masiello, A., Multiple critical points for indefinite functionals and applications, J. Differential Equations 155 (1999), 203-230.

[26] Candela, A.M., Masiello, A., Salvatore, A., Existence and multiplicity of normal geodesics in Lorentzian manifolds, J. Geom. Anal. 10 (2000), 623-651.

[27] Candela, A.M., Salvatore, A., Closed geodesics in Riemannian manifolds with convex boundary, Proc. Roy. Soc. Edinburgh 124A (1994), 1247-1258.

[28] Candela, A.M., Salvatore, A., Closed geodesics in stationary manifolds with strictly convex boundary, Differential Geom. Appl. 13 (2000), 251-266.

[29] Candela, A.M., Sánchez, M., Geodesic connectedness in Gödel type space-times, Differential Geom. Appl. 12 (2000), 105-120.

[30] Caponio, E., Masiello, A., Piccione, P., Some global properties of static spacetimes, Math. Z. 244 (2003), 457-468.

[31] Carrière, Y., Autour de la conjecture de L. Markus sur les variétés affines, Invent. Math. 95 (1989), 615-628.

[32] Carrière, Y., Rozoy, L., Complétude des métriques lorentziennes de $T^{2}$ et difféomorphismes du cercle, Bol. Soc. Bras. mat., Nova Sér. 25 (1994), 223-235.

[33] Cerami, G., Un criterio di esistenza per i punti critici su varietà illimitate, Istit. Lombardo Accad. Sci. Lett. Rend. A 112 (1978), 332-336.

[34] Charlap, L.S., Bieberbach Groups and Flat Manifolds, Springer-Verlag, New York, 1986. 
[35] Fadell, E., Lectures in Cohomological Index Theories of G-spaces with Applications to Critical Point Theory, Raccolta di seminari, Università della Calabria, 1985.

[36] Fadell, E., Husseini, S., Category of loop spaces of open subsets in Euclidean space, Nonlinear Anal. TMA 17 (1991), 1153-1161.

[37] Fadell, E., Husseini, S., Relative category, products and coproducts, Rend. Sem. Mat. Fis. Univ. Milano LXIV (1994), 99-117.

[38] Flores, J.L., Sánchez, M., Geodesics in stationary spacetimes. Application to Kerr spacetime, Int. J. Theor. Phys. Group Theory Nonlinear Opt. 8 (2002), 319-336.

[39] Flores, J.L., Sánchez, M., Geodesic connectedness of multiwarped spacetimes, J. Differential Equations 186 (2002), 1-30.

[40] Flores, J.L., Sánchez, M., A topological method for geodesic connectedness: outer Kerr spacetime, J. Math. Phys. 43 (2002), 4861-4885.

[41] Fortunato, D., Giannoni, F., Masiello, A., A Fermat principle for stationary spacetimes and applications to light rays, J. Geom. Phys. 15 (1995), 159-188.

[42] Fortunato, D., Masiello, A., Fermat principles in General Relativity and the existence of light rays on Lorentzian manifolds. In: Proceedings "Variational and Local Methods in the Study of Hamiltonian Systems", World Scientific, Singapore (1995), 34-64.

[43] Fournier, G., Willem, M., Relative category and the calculus of variations. In: Variational Problems (H. Beresticky, J.M. Coron, I. Ekeland Eds), Birkhäuser, Basel (1990), 95-104.

[44] Fried, D., Goldman, W., Hirsch, M.W., Affine manifolds with nilpotent holonomy, Comment. Math. Helv. 56 (1981), 487-523.

[45] Furness, P.M.D., Arrowsmith, D.K., Locally symmetric spaces, J. London Math. Soc. (2) 10 (1975), 487-499.

[46] Furness, P.M.D., Fedida, E., Sur les structures pseudo-riemanniennes plates des variétés compactes, J. Nigerian Math. Soc. 5 (1986), 63-78 (1989).

[47] Giannoni, F., Masiello, A., On the existence of geodesics on stationary Lorentz manifolds with convex boundary, J. Funct. Anal. 101 (1991), 340-369.

[48] Giannoni, F., Masiello, A., Geodesics on product Lorentzian manifolds, Ann. Inst. H. Poincaré Anal. Non Linéaire 12 (1995), 27-60.

[49] Giannoni, F., Piccione, P., An intrinsic approach to the geodesical connectedness of stationary Lorentzian manifolds, Comm. Anal. Geom. 7 (1999), 157-197.

[50] Greco, C., Periodic trajectories in static space-times, Proc. Roy. Soc. Edinburgh 113A (1989), 99-103.

[51] Gromoll, D., Meyer, W., Periodic geodesics on compact Riemannian manifolds, J. Differential Geom. 3 (1969), 493-510.

[52] Guediri, M., Lafontaine, J., Sur la complétude des variétés pseudo-Riemanniennes, J. Geom. Phys. 15 (1995), 150-158.

[53] Hawking, S.W., Ellis, G.F.R., The Large Scale Structure of Space-Time, Cambridge University Press, Cambridge, 1973.

[54] Helfer, A.D., Conjugate points on spacelike geodesics or pseudo-selfadjoint MorseSturm-Liouville systems, Pacific J. Math. 164 (1994), 321-350. 
[55] Hopf, H., Rinow, W., Über den Begriff des vollständigen differentialgeometrischen Fläche, Comment. Math. Helv. 3 (1931), 209-225.

[56] Klingler, B., Complétude des variétés lorentziennes à courbure constante, Math. Ann. 306 (1996), 353-370.

[57] Kobayashi, S., Nomizu, K., Foundations of Differential Geometry (Reprint of the 1969 original), Wiley \& Sons Inc., New York, 1996.

[58] Lafuente López, J., A geodesic completeness theorem for locally symmetric Lorentz manifolds, Rev. Mat. Complut. 1 (1988), 101-110.

[59] Marsden, J.E., On completeness of homogeneous pseudo-Riemannian manifolds, Indiana Univ. J. 22 (1972/73), 1065-1066.

[60] Masiello, A., On the existence of a closed geodesic in stationary Lorentz manifolds, J. Differential Equations 104 (1993), 48-59.

[61] Masiello, A., Variational Methods in Lorentzian Geometry, Pitman Res. Notes Math. Ser. 309, Longman Sci. Tech., Harlow, 1994.

[62] Minguzzi, E., Sánchez, M., Connecting solutions of the Lorentz force equation do exist, Comm. Math. Phys. 264 (2006), 349-370. Erratum 267 (2006), 559-561.

[63] Minguzzi, E., Sánchez, M., The causal hierarchy of spacetimes. In this proceedings.

[64] Misner, C.W., Taub-NUT space as a counterexample to almost anything. In: Relativity Theory and Astrophysics I: Relativity and Cosmology (J. Ehlers Ed.), Lectures in Applied Mathematics 8 A.M.S. (1967), 160-169.

[65] Molina, J., Existence and multiplicity of normal geodesics on static space-time manifolds, Bull. Un. Mat. Ital. A 10 (1996), 305-318.

[66] Nash, J., The imbedding problem for Riemannian manifold, Ann. Math. 63 (1956), 20-63.

[67] O'Neill, B., Semi-Riemannian Geometry with Applications to Relativity, Academic Press Inc., New York, 1983.

[68] Palais, R.S., Morse theory on Hilbert manifolds, Topology 2 (1963), 299-340.

[69] Palais, R.S., Lusternik-Schnirelman Theory on Banach manifolds, Topology 5 (1966), 115-132.

[70] Palomo, F.J., Romero, A., Certain actual topics on modern Lorentzian geometry. In: Handbook of Differential Geometry. Vol. II, Elsevier/North-Holland (2006), 513-546.

[71] Penrose, R., Techniques of Differential Topology in Relativity, Conf. Board Math. Sci. 7, S.I.A.M., Philadelphia (1972).

[72] Piccione, P., Sampalmieri, R., Geodesical connectedness of compact Lorentzian manifolds, Dynamic Systems Appl. 5 (1996), 479-502.

[73] Piccione, P., Portaluri, A., Tausk, D.V., Spectral flow, Maslov index and bifurcation of semi-Riemannian geodesics, Ann. Global Anal. Geom. 25 (2004), 121-149.

[74] Piccione, P., Tausk, D.V., The Morse index theorem in semi-Riemannian geometry, Topology 41 (2002), 1123-1159.

[75] Piccione, P., Tausk, D.V., On the distribution of conjugate points along semiRiemannian geodesics, Comm. Anal. Geom. 11 (2003), 33-48.

[76] Rabinowitz, P.H., Minimax Methods in Critical Point Theory with Applications to Differential Equations, CBMS Reg. Conf. Ser. Math. 65, Providence, 1986. 
[77] Romero, A., Sánchez, M., On the completeness of geodesics obtained as a limit, J. Math. Phys. 34 (1993), 3768-3774.

[78] Romero, A., Sánchez, M., New properties and examples of incomplete Lorentzian tori, J. Math. Phys. 35 (1994), 1992-1997.

[79] Romero, A., Sánchez, M., On completeness of certain families of semi-Riemannian manifolds, Geom. Dedicata 53 (1994), 103-117.

[80] Romero, A., Sánchez, M., Completeness of compact Lorentz manifolds admitting a timelike conformal Killing vector field, Proc. Amer. Math. Soc. 123 (1995), 2831-2833.

[81] Sánchez, M., An introduction to the completeness of compact semi-Riemannian manifolds, Sémin. Théor. Spectr. Géom. 13 Univ. Grenoble I, Saint-Martin-d'Hères (1995), 37-53.

[82] Sánchez, M., Some remarks on causality theory and variational methods in Lorentzian manifolds, Conf. Semin. Mat. Univ. Bari 265 (1997).

[83] Sánchez, M., Geodesic connectedness in generalized Reissner-Nordstrm type Lorentz manifolds, Gen. Relativity Gravitation 29 (1997), 1023-1037.

[84] Sánchez, M., Structure of Lorentzian tori with a Killing vector field, Trans. Amer. Math. Soc. 349 (1997), 1063-1080.

[85] Sánchez, M., On the geometry of generalized Robertson-Walker spacetimes: geodesics, Gen. Relativity Gravitation 30 (1998), 915-932.

[86] Sánchez, M., Geodesics in static spacetimes and $t$-periodic trajectories, Nonlinear Anal. TMA 35 (1999), 677-686.

[87] Sánchez, M., On the geometry of generalized Robertson-Walker spacetimes: curvature and Killing fields, J. Geom. Phys. 31 (1999), 1-15.

[88] Sánchez, M., Timelike periodic trajectories in spatially compact Lorentz manifolds, Proc. Amer. Math. Soc. 127 (1999), 3057-3066.

[89] Sánchez, M., Geodesic connectedness of semi-Riemannian manifolds, Nonlinear Anal. TMA 47 (2001), 3085-3102.

[90] Sánchez, M., On causality and closed geodesics of compact Lorentzian manifolds and static spacetimes, Differential Geom. Appl. 24 (2006), 21-32.

[91] Senovilla, J.M.M., Singularity theorems and their consequences, Gen. Relativity Gravitation 29 (1997), 701-848.

[92] Smith, J., Lorentz structures on the plane, Trans. Amer. Math. Soc. 95 (1960), 226-237.

[93] Struwe, M., Variational Methods. Applications to Nonlinear Partial Differential Equations and Hamiltonian Systems, 3rd Edition, Springer, Berlin, 1996.

[94] Szulkin, A., A relative category and applications to critical point theory for strongly indefinite functionals, Nonlinear Anal. TMA 15 (1990), 725-739.

[95] Uhlenbeck, K., A Morse theory for geodesics on a Lorentz manifold, Topology 14 (1975), 69-90.

[96] Unal, B., Doubly warped products, Differential Geom. Appl. 15 (2001), 253-263.

[97] Unal, B., Multiply warped products, J. Geom. Phys. 34 (2000), 287-301.

[98] Weinstein, T., An Introduction to Lorentz Surfaces, De Gruyter Exp. Math., New York, 1996. 
[99] Yurtsever, U., A simple proof of geodesical completeness for compact space-times of zero curvature, J. Math. Phys. 33 (1992), 1295-1300.

Anna Maria Candela,

Dipartimento di Matematica,

Università degli Studi di Bari,

Via E. Orabona 4, 70125 Bari, Italy

E-mail: candela@dm.uniba.it

Miguel Sánchez,

Departamento de Geometría y Topología, Facultad de Ciencias, Universidad de Granada,

Avenida Fuentenueva s/n, 18071 Granada, Spain

E-mail: sanchezm@ugr.es 